PHYSICAL REVIEW D 94, 102002 (2016)

\title{
Results of the deepest all-sky survey for continuous gravitational waves on LIGO S6 data running on the Einstein@Home volunteer distributed computing project
}

\author{
B. P. Abbott et al. \\ (LIGO Scientific Collaboration and Virgo Collaboration) \\ (Received 6 July 2016; published 18 November 2016)
}

\begin{abstract}
We report results of a deep all-sky search for periodic gravitational waves from isolated neutron stars in data from the S6 LIGO science run. The search was possible thanks to the computing power provided by the volunteers of the Einstein@Home distributed computing project. We find no significant signal candidate and set the most stringent upper limits to date on the amplitude of gravitational wave signals from the target population. At the frequency of best strain sensitivity, between 170.5 and $171 \mathrm{~Hz}$ we set a $90 \%$ confidence upper limit of $5.5 \times 10^{-25}$, while at the high end of our frequency range, around $505 \mathrm{~Hz}$, we achieve upper limits $\simeq 10^{-24}$. At $230 \mathrm{~Hz}$ we can exclude sources with ellipticities greater than $10^{-6}$ within $100 \mathrm{pc}$ of Earth with fiducial value of the principal moment of inertia of $10^{38} \mathrm{~kg} \mathrm{~m}^{2}$. If we assume a higher (lower) gravitational wave spin-down we constrain farther (closer) objects to higher (lower) ellipticities.
\end{abstract}

DOI: 10.1103/PhysRevD.94.102002

\section{INTRODUCTION}

In this paper we report the results of a deep all-sky Einstein@Home [1] search for continuous, nearly monochromatic gravitational waves (GWs) in data from LIGO's sixth science (S6) run. A number of all-sky searches have been carried out on LIGO data, [2-11], of which [5,7,10] also ran on Einstein@Home. The search presented here covers frequencies from $50 \mathrm{~Hz}$ through $510 \mathrm{~Hz}$ and frequency derivatives from $3.39 \times 10^{-10} \mathrm{~Hz} / \mathrm{s}$ through $-2.67 \times 10^{-9} \mathrm{~Hz} / \mathrm{s}$. In this range we establish the most constraining gravitational wave amplitude upper limits to date for the target signal population.

\section{LIGO INTERFEROMETERS AND THE DATA USED}

The LIGO gravitational wave network consists of two observatories, one in Hanford (WA) and the other in Livingston (LA) separated by a $3000-\mathrm{km}$ baseline [12]. The last science run (S6) [13] of this network before the upgrade towards the advanced LIGO configuration [14] took place between July 2009 and October 2010. The analysis in this paper uses a subset of this data: from GPS 949469977 (2010 Feb 6 05:39:22 UTC) through GPS 971529850 (2010 Oct 19 13:23:55 UTC), selected for good strain sensitivity [15]. Since interferometers sporadically fall out of operation ("lose lock") due to environmental or

${ }^{*}$ Full author list given at end of the article.

Published by the American Physical Society under the terms of the Creative Commons Attribution 3.0 License. Further distribution of this work must maintain attribution to the author(s) and the published article's title, journal citation, and DOI. instrumental disturbances or for scheduled maintenance periods, the data set is not contiguous and each detector has a duty factor of about 50\% [16].

As done in [7], frequency bands known to contain spectral disturbances have been removed from the analysis. Actually, the data has been substituted with Gaussian noise with the same average power as that in the neighboring and undisturbed bands. Table III identifies these bands.

\section{THE SEARCH}

The search described in this paper targets nearly monochromatic gravitational wave signals as described for example by Eqs. 1-4 of [7]. Various emission mechanisms could generate such a signal as reviewed in Sec. II A of [11]. In interpreting our results we will consider a spinning compact object with a fixed, nonaxisymmetric mass quadrupole, described by an ellipticity $\epsilon$.

We perform a stack-slide type of search using the GCT (Global Correlation Transform) method [17,18]. In a stackslide search the data is partitioned in segments and each segment is searched with a matched-filter method [19]. The results from these coherent searches are combined by summing (stacking) the detection statistic values from the segments (sliding), one per segment $\left(\mathcal{F}_{i}\right)$, and this determines the value of the core detection statistic:

$$
\overline{\mathcal{F}}:=\frac{1}{N_{\text {seg }}} \sum_{i=1}^{N_{\text {seg }}} \mathcal{F}_{i}
$$

There are different ways to combine the single-segment $\mathcal{F}_{i}$ values, but independently of the way that this is done, this type of search is usually referred to as a "semicoherent 


\section{B. P. ABBOTT et al.}

search". So stack-slide searches are a type of semicoherent search. Important variables for this type of search are the coherent time baseline of the segments $T_{\text {coh }}$, the number of segments used $N_{\text {seg }}$, the total time spanned by the data $T_{\text {obs }}$, the grids in parameter space and the detection statistic used to rank the parameter space cells. For a stack-slide search in Gaussian noise, $N_{\text {seg }} \times 2 \overline{\mathcal{F}}$ follows a $\chi_{4 N_{\text {seg }}}^{2}$ chi-squared distribution with $4 N_{\text {seg }}$ degrees of freedom. These parameters are summarized in Table I. The grids in frequency and spin-down are each described by a single parameter, the grid spacing, which is constant over the search range. The same frequency grid spacings are used for the coherent searches over the segments and for the incoherent summing. The spin-down spacing for the incoherent summing, $\delta \dot{f}$, is finer than that used for the coherent searches, $\delta \dot{f}_{c}$, by a factor $\gamma$. The notation used here is consistent with that used in previous observational papers [20] and in the GCT methods papers cited above.

The sky grid is the union of two grids: one is uniform over the projection of the celestial sphere onto the equatorial plane, and the tiling (in the equatorial plane) is approximately square with sides of length

$$
d\left(m_{\mathrm{sky}}\right)=\frac{1}{f} \frac{\sqrt{m_{\mathrm{sky}}}}{\pi \tau_{E}},
$$

with $m_{\text {sky }}=0.3$ and $\tau_{E} \simeq 0.021 \mathrm{~s}$ being half of the light travel time across the Earth. As was done in [7], the skygrids are constant over $10 \mathrm{~Hz}$ bands and the spacings are the ones associated through Eq. (2) to the highest frequency $f$ in the range. The other grid is limited to the equatorial region $(0 \leq \alpha \leq 2 \pi$ and $-0.5 \leq \delta \leq 0.5)$, with constant right ascension $\alpha$ and declination $\delta$ spacings equal to $d(0.3)$ - see Fig. 1. The reason for the equatorial "patching" with a denser sky grid is to improve the sensitivity of the search: the sky resolution actually depends on the ecliptic latitude and the uniform equatorial grid underresolves particularly in the equatorial region. The resulting number of templates used to search $50 \mathrm{mHz}$ bands as a function of frequency is shown in Fig. 2.

The search is split into work-units (WUs) sized to keep the average Einstein@Home volunteer computer busy for about six hours. Each WU searches a $50 \mathrm{mHz}$ band, the

TABLE I. Search parameters rounded to the first decimal figure. $T_{\text {ref }}$ is the reference time that defines the frequency and frequency derivative values.

\begin{tabular}{lc}
\hline \hline$T_{\text {coh }}$ & 60 hours \\
$T_{\text {ref }}$ & $960499913.5 \mathrm{GPS} \mathrm{sec}$ \\
$N_{\text {seg }}$ & 90 \\
$\delta f$ & $1.6 \times 10^{-6} \mathrm{~Hz}$ \\
$\delta \dot{f}_{c}$ & $5.8 \times 10^{-11} \mathrm{~Hz} / \mathrm{s}$ \\
$\gamma$ & 230 \\
$m_{\text {sky }}$ & $0.3+$ equatorial patch \\
\hline \hline
\end{tabular}
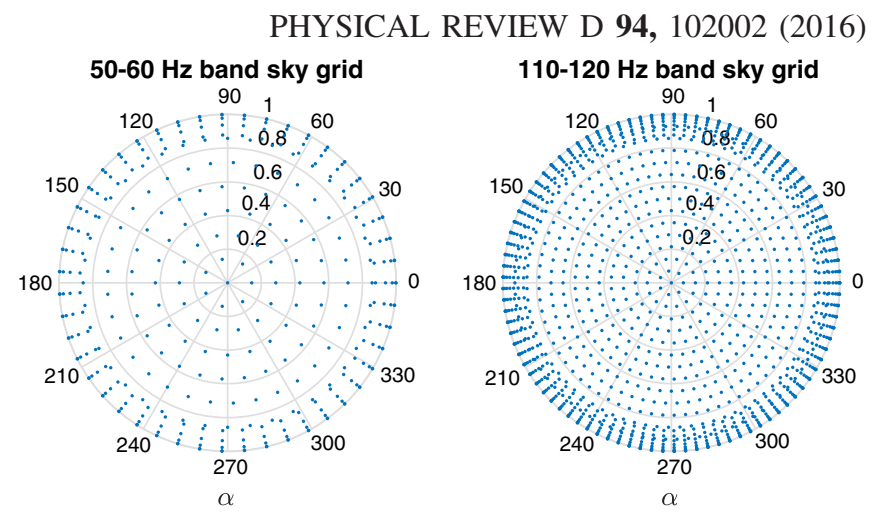

FIG. 1. Polar plots ( $r, \theta$ plots with $\theta=\alpha$ and $r=\cos \delta$ ) of the grid points in the northern equatorial hemisphere sky for the band $50-60 \mathrm{~Hz}$ (left panel) and for the band $110-120 \mathrm{~Hz}$ (right panel). $\alpha$ is the right ascension coordinate and $\delta$ the declination coordinate. One can clearly see the higher density in the $-0.5 \leq \delta \leq 0.5$ equatorial region and the higher density $\left(\propto f^{2}\right)$ of grid points at higher frequencies. The southern hemispheres looks practically identical to the respective northern ones.

entire spin-down range and 13 points in the sky, corresponding to $4.9 \times 10^{9}$ templates out of which it returns only the top 3000. A total of 12.7 million WUs are necessary to cover the entire parameter space. The total number of templates searched is $6.3 \times 10^{16}$.

\section{A. The ranking statistic}

The search was actually carried out in separate Einstein@Home runs that used different ranking statistics to define the top-candidate-list, reflecting different stages in the development of a detection statistic robust with respect

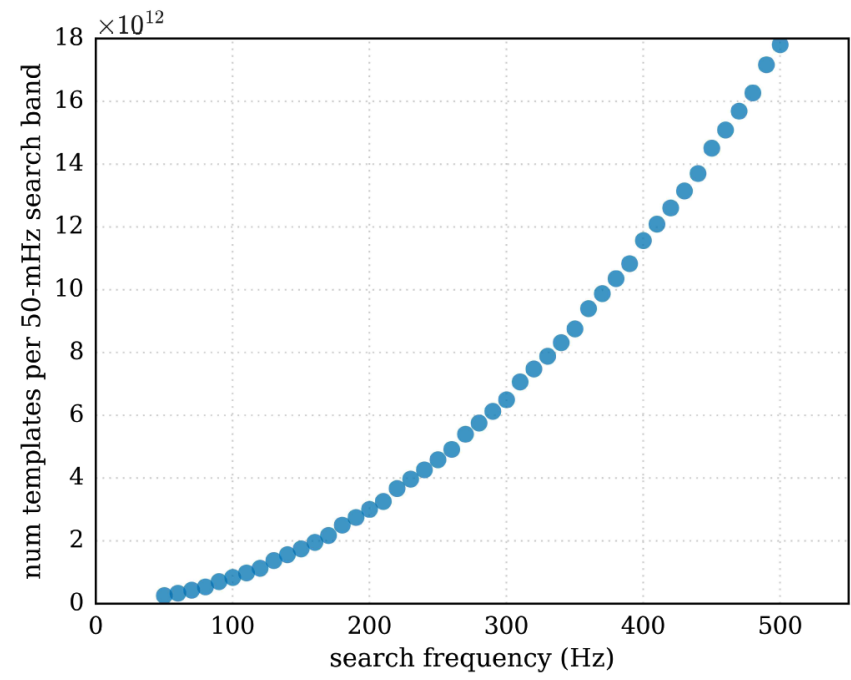

FIG. 2. Number of searched templates in $50 \mathrm{mHz}$ bands. The variation with frequency is due to the increasing sky resolution. $N_{f} \times N_{\dot{f}} \sim 3.7 \times 10^{8}$, where $N_{f}$ and $N_{\dot{f}}$ are the number of $f$ and $\dot{f}$ templates searched in $50 \mathrm{mHz}$ bands. The total number of templates searched between 50 and $510 \mathrm{~Hz}$ is $6.3 \times 10^{16}$. 
to spectral lines in the data [21]. In particular, three ranking statistics were used: the average $2 \mathcal{F}$ statistic over the segments, $2 \overline{\mathcal{F}}$, which in essence at every template point is the likelihood of having a signal with the shape given by the template versus having Gaussian noise; the line-veto statistic $\hat{O}_{\mathrm{SL}}$ which is the odds ratio of having a signal versus having a spectral line; and a general line-robust statistic, $\hat{O}_{\mathrm{SGL}}$, that tests the signal hypothesis against a Gaussian noise + spectral line noise model. Such a statistic can match the performance of both the standard average $2 \overline{\mathcal{F}}$ statistic in Gaussian noise and the line-veto statistic in presence of single-detector spectral disturbances and statistically outperforms them when the noise is a mixture of both [21].

We combine the $2 \overline{\mathcal{F}}$-ranked results with the $\hat{O}_{\mathrm{SL}}$-ranked results to produce a single list of candidates ranked according to the general line-robust statistic $\hat{O}_{\mathrm{SGL}}$. We now explain how this is achieved. Alongside the detection statistic value and the parameter space cell coordinates of each candidate, the Einstein@Home application also returns the single-detector $2 \overline{\mathcal{F}}^{X}$ values (" $X$ " indicates the detector). These are used to compute, for every candidate of any run, the $\hat{O}_{\mathrm{SGL}}$ through Eq. 61 of [21]

$$
\begin{aligned}
\ln \hat{O}_{\mathrm{SGL}}= & \ln \hat{o}_{\mathrm{SL}}+\hat{\mathcal{F}}-\hat{\mathcal{F}}_{\text {max }}^{\prime \prime} \\
& -\ln \left(\mathrm{e}^{\hat{\mathcal{F}}_{*}-\hat{\mathcal{F}}_{\text {max }}^{\prime \prime}}+\left\langle\hat{r}^{X} \mathrm{e}^{\left.\left.\hat{\mathcal{F}}^{X}-\hat{\mathcal{F}}_{\text {max }}^{\prime \prime}\right\rangle\right),}\right.\right.
\end{aligned}
$$

with the angle-brackets indicating the average with respect to detectors $(X)$ and

$$
\begin{gathered}
\hat{\mathcal{F}}=N_{\text {seg }} \overline{\mathcal{F}} \\
\hat{\mathcal{F}}^{X}=N_{\text {seg }} \overline{\mathcal{F}}^{X} \\
\hat{\mathcal{F}}_{\max }^{\prime \prime} \equiv \max \left(\hat{\mathcal{F}}_{*}, \hat{\mathcal{F}}^{X}+\ln \hat{r}^{X}\right) \\
\hat{\mathcal{F}}_{*} \equiv \hat{\mathcal{F}}_{*}^{(0)}-\ln \hat{o}_{\mathrm{LG}} \\
\hat{\mathcal{F}}_{*}^{(0)} \equiv \ln c_{*}^{N_{\text {seg }}} \quad \text { with } \quad c_{*} \text { set to } 20.64 \\
\hat{o}_{\mathrm{LG}}=\sum_{X} \hat{o}_{\mathrm{LG}}^{X} \\
\hat{r}^{X} \equiv \frac{\hat{o}_{\mathrm{LG}}^{X}}{\hat{o}_{\mathrm{LG}} / N_{\mathrm{det}}} \\
\hat{p}_{\mathrm{L}} \equiv \frac{\hat{o}_{\mathrm{LG}}}{1+\hat{o}_{\mathrm{LG}}},
\end{gathered}
$$

where $\hat{o}_{\mathrm{LG}}^{X}$ is the assumed prior probability of a spectral line occurring in any frequency bin of detector $\mathrm{X}, \hat{p}_{\mathrm{L}}$ is the line prior estimated from the data, $N_{\text {det }}=2$ is the number of detectors, and $\hat{o}_{\mathrm{SL}}$ is an assumed prior probability of a line being a signal (set arbitrarily to 1 ; its specific value does not affect the ranking statistic). Following the reasoning of Eq. 67 of [21], with $N_{\text {seg }}=90$ we set $c_{*}=20.64$ corresponding to a Gaussian false-alarm probability of $10^{-9}$ and an average $2 \overline{\mathcal{F}}$ transition scale of $\sim 6\left(\mathcal{F}_{*}^{(0)} \sim 3\right)$. The $\hat{o}_{\mathrm{LG}}^{X}$ values are estimated from the data as described in Sec. VI. A of [21] in 50-mHz bands with a normalized-SFT-power threshold $\mathcal{P}_{\text {thr }}^{X}=\mathcal{P}_{\text {thr }}\left(p_{\mathrm{FA}}=10^{-9}, N_{\mathrm{SFT}}^{X} \sim 6000\right) \approx 1.08$. For every $50 \mathrm{mHz}$ band the list of candidates from the $2 \overline{\mathcal{F}}$-ranked run is merged with the list from the $\hat{O}_{\mathrm{SL}}$-ranked run and duplicate candidates are considered only once. The resulting list is ranked by the newly computed $\hat{O}_{\mathrm{SGL}}$ and the top 3000 candidates are kept. This is our result-set, and it is treated in a manner that is very similar to [3].

\section{B. Identification of undisturbed bands}

Even after the removal of disturbed data caused by spectral artifacts of known origin, the statistical properties of the results are not uniform across the search band. In what follows we concentrate on the subset of the signalfrequency bands having reasonably uniform statistical properties. This still leaves us with the majority of the search parameter space while allowing us to use methods that rely on theoretical modeling of the significance in the statistical analysis of the results. Our classification of "clean" vs "disturbed" bands has no pretence of being strictly rigorous, because strict rigor here is neither useful nor practical. The classification serves the practical purpose of discarding from the analysis regions in parameter space with evident disturbances and must not dismiss real signals. The classification is carried out in two steps: a visual inspection and a refinement on the visual inspection.

The visual inspection is performed by three scientists who each look at various distributions of the detection statistics over the entire sky and spin-down parameter space in $50 \mathrm{mHz}$ bands. They rank each band with an integer score $0,1,2,3$ ranging from "undisturbed" $(0)$ to "disturbed" (3). A band is considered "undisturbed" if all three rankings are 0 . The criteria agreed upon for ranking are that the distribution of detection statistic values should not show a visible trend affecting a large portion of the $f-\dot{f}$ plane and, if outliers exist in a small region, outside this region the detection statistic values should be within the expected ranges. Figure 3 shows the $\hat{O}_{\mathrm{SGL}}$ for three bands: two were marked as undisturbed and the other as disturbed. One of the bands contains the $f-\dot{f}$ parameter space that harbors a fake signal injected in the data to verify the detection pipelines. The detection statistic is elevated in a small region around the signal parameters. The visual inspection procedure does not mark as disturbed bands with such features.

Based on this visual inspection $13 \%$ of the bands between 50 and $510 \mathrm{~Hz}$ are marked as "disturbed". Of these, $34 \%$ were given by all visual inspectors rankings 

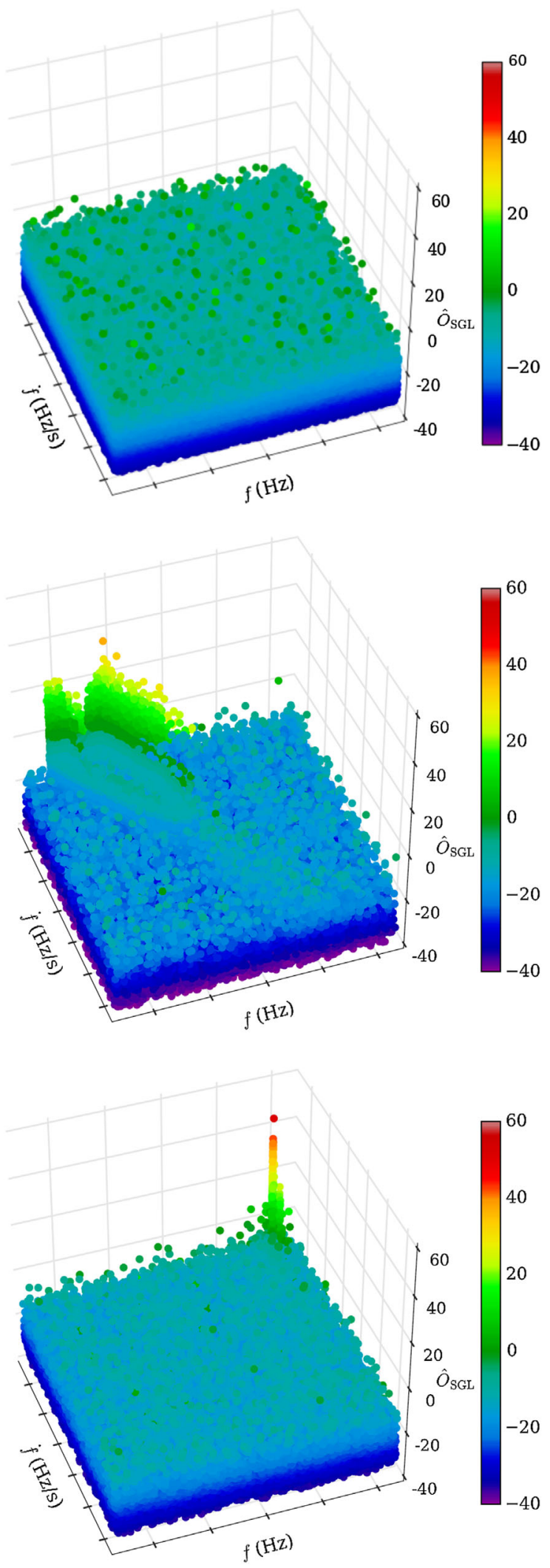

FIG. 3. On the z-axis and color-coded is the $\hat{O}_{\mathrm{SGL}}$ in three $50 \mathrm{mHz}$ bands. The top band was marked as "undisturbed". The middle band is an example of a "disturbed band". The bottom band is an example of an "undisturbed band" but containing a signal, a fake one, in this case. smaller than 3 , i.e. they were only marginally disturbed. Further inspection "rehabilitated" $42 \%$ of these. As a result of this refinement in the selection procedure we exclude from the current analysis $11 \%$ of the searched frequencies (Table IV).

Figure 4 shows the highest values of the detection statistic in half-Hz signal-frequency bands compared to the expectations. The set of candidates that the highest detection statistic values are picked from, does not include the $50 \mathrm{mHz}$ signal-frequency bands that stem entirely from fake data, from the cleaning procedure, or that were marked as disturbed. In this paper we refer to the candidates with the highest value of the detection statistic as the loudest candidates.

The loudest expected value over $N_{\text {trials }}$ independent trials of $2 \overline{\mathcal{F}}$ is determined ${ }^{1}$ by numerical integration of the probability density function given, for example, by Eq. 7 of [20]. For this search, we estimate that $N_{\text {trials }} \simeq 0.87 N_{\text {templ }}$, with $N_{\text {templ }}$ being the number of templates searched.

As a uniform measure of significance of the highest $2 \overline{\mathcal{F}}$ value across bands that were searched with different values of $N_{\text {trials }}$ we introduce the critical ratio CR defined as the deviation of the measured highest $2 \overline{\mathcal{F}}$ from the expected value, measured in units of the standard deviation

$$
\mathrm{CR}:=\frac{2 \overline{\mathcal{F}}_{\text {meas }}-2 \overline{\mathcal{F}}_{\text {exped }}}{\sigma_{\text {exped }}} .
$$

The highest and most significant detection statistic value from our search is $2 \overline{\mathcal{F}}=8.6$ at a frequency of about $52.76 \mathrm{~Hz}$ with a $\mathrm{CR}=29$. This is due to a fake signal. The second highest value of the detection statistic is 7.04 at a frequency of about $329.01 \mathrm{~Hz}$ corresponding to a CR of 4.6. The second highest-CR candidate has a $2 \overline{\mathcal{F}}$ of 6.99 , is

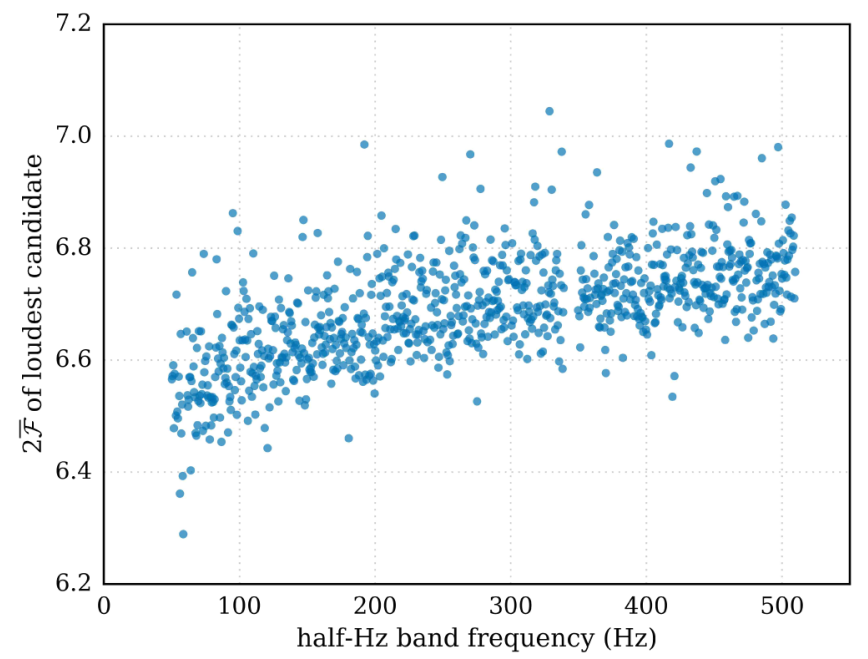

FIG. 4. Highest values of $2 \overline{\mathcal{F}}$ in every half-Hz band as a function of band frequency. Since the number of templates increases with frequency so does the loudest $2 \overline{\mathcal{F}}$. 


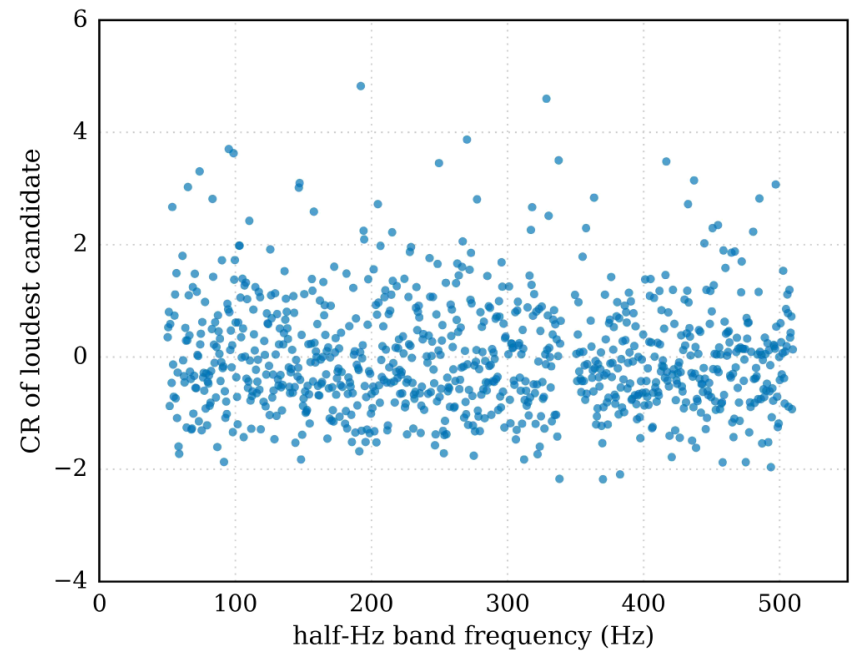

FIG. 5. Highest values of the significance (CR) in every half-Hz band as a function of band frequency. Since the significance folds in the expected value for the loudest $2 \overline{\mathcal{F}}$ and its standard deviation, the significance of the loudest in noise does not increase with frequency. Our results are consistent with this expectation.

at $192.16 \mathrm{~Hz}$ and has a $\mathrm{CR}=4.8$. The $\mathrm{CR}$ values are plotted in Fig. 5, and the distribution in Fig. 6.

Sorting loudest candidates from half-Hz bands according to detection statistic values is not the same as sorting them according to $\mathrm{CR}$. The reason for this is that the number of templates is not the same for all half-Hz bands. This is due to the grid spacings decreasing with frequency (Eq. (2) and to the fact that, as previously explained, some $50 \mathrm{mHz}$ bands have been excluded from the current analysis and hence some half- $\mathrm{Hz}$ bands comprise results from fewer than ten $50 \mathrm{mHz}$ bands. Figure 7 gives the fill-level of each half-Hz band, i.e. how many $50 \mathrm{mHz}$ bands have contributed candidates to the analysis out of ten. We use the CR as

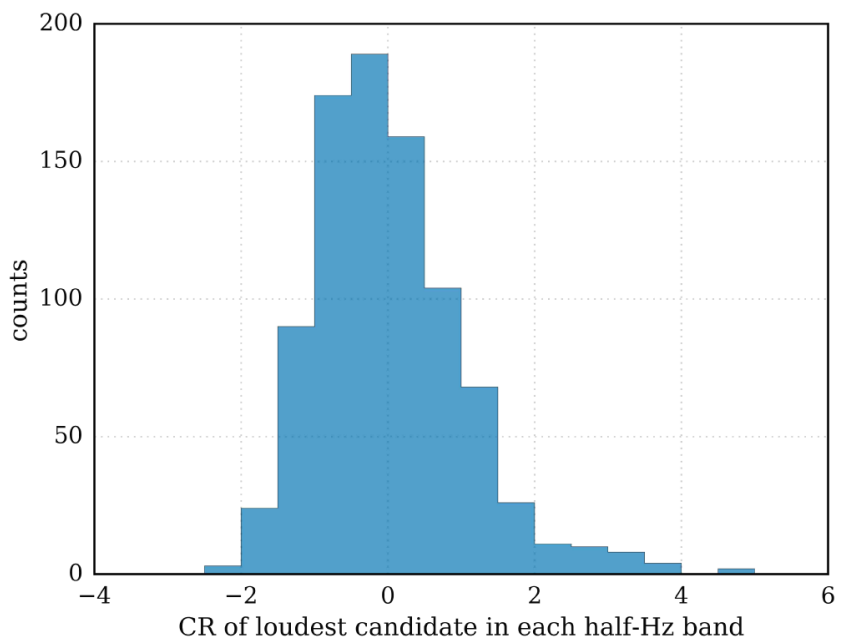

FIG. 6. Histogram of the highest values of the significance CR in every half-Hz band.

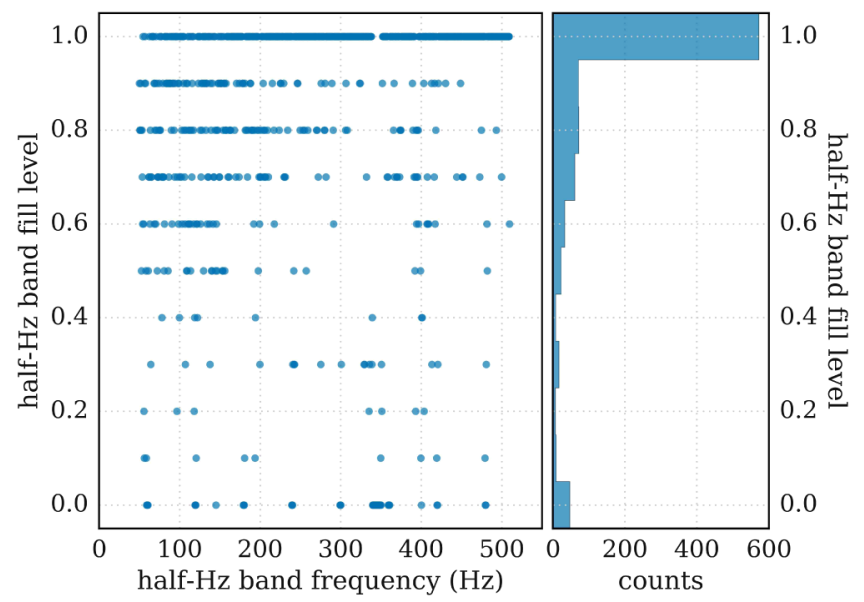

FIG. 7. The fraction of $50 \mathrm{mHz}$ bands (in signal frequency) which contribute to the results in every half- $\mathrm{Hz}$ band. As explained in the text, some bands are excluded because they are all from fake data or because they are marked as disturbed by the visual inspection. The list of excluded bands is given in Table IV.

a measure of the significance because it folds in correctly the effect of varying number of templates in the half$\mathrm{Hz}$ bands.

After excluding the candidate due to the fake signal, in this data we see no evidence of a signal: the distribution of $p$ values associated with every measured half- $\mathrm{Hz}$ band loudest is consistent with what we expect from noise-only across the measured range (Fig. 8). In particular we note two things: 1) the two candidates at $\mathrm{CR}=4.6$ and $\mathrm{CR}=4.8$ are not significant when we consider how many half-Hz bands we have searched, and 2) there is no population of low significance candidates deviating from the expectation of the noise-only case. The $p$ value for the loudest measured in any half- $\mathrm{Hz}$ band searched with an

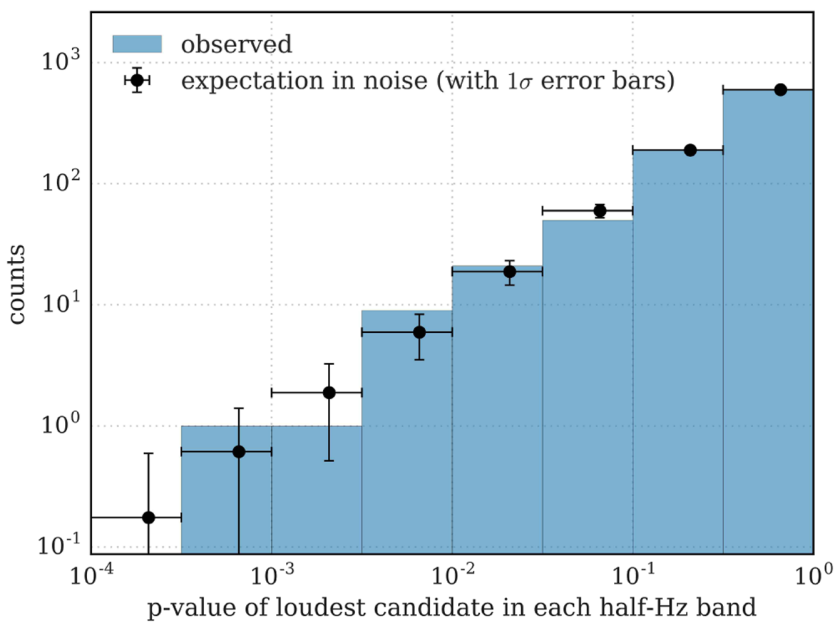

FIG. 8. p values for the loudest in half- $\mathrm{Hz}$ bands of our data (histogram bars) and expected distribution of pure noise data for reference (black markers). 
effective number of independent trials $N_{\text {trials }}=0.87 N_{\text {trials }}$ is obtained by integrating Eq. 6 of [20] between the observed value and infinity.

\section{UPPER LIMITS}

The search did not reveal any continuous gravitational wave signal in the parameter volume that was searched. We hence set frequentist upper limits on the maximum gravitational wave amplitude consistent with this null result in half-Hz bands: $h_{0}^{90 \%}(\mathrm{f}) . h_{0}^{90 \%}(\mathrm{f})$ is the GW amplitude such that $90 \%$ of a population of signals with parameter values in our search range would have produced a candidate louder than what was observed by our search. This is the criterion hereafter referred to as "detection".

Evaluating these upper limits with injection-andrecovery Monte Carlo simulations in every half- $\mathrm{Hz}$ band is too computationally intensive. So we perform them in a subset of 50 bands and infer the upper limit values in the other bands from these. The 50 bands are evenly spaced in the search frequency range. For each band $j=1 \ldots 50$, we measure the $90 \%$ upper limit value corresponding to different detection criteria. The different detection criteria are defined by different $\mathrm{CR}$ values for the assumed measured loudest. The first $\mathrm{CR}$ bin, $\mathrm{CR}_{0}$, is for $\mathrm{CR}$ values equal to or smaller than 0 , the next bins are for $i<\mathrm{CR}_{i} \leq$ $(i+1)$ with $i=1 \ldots 5$. Correspondingly we have $h_{0, \mathrm{CR}_{i}}^{90 \%, j}$ for each band. For every detection criteria and every band we determine the sensitivity depth [22], and by averaging these sensitivity depths over the bands we derive a sensitivity depth for every detection criteria: $\mathcal{D}_{\mathrm{CR}_{i}}^{90 \%}=1 / 50 \sum_{j} \mathcal{D}_{\mathrm{CR}_{i}}^{90 \%,}$. We use these to set upper limits in the bands $k$ where we have not performed injection-and-recovery simulations as

$$
h_{0}^{90 \%}\left(f_{k}\right)=\frac{\sqrt{S_{h}\left(f_{k}\right)}}{\mathcal{D}_{\mathrm{CR}_{i}(k)}^{90 \%}},
$$

where $\mathrm{CR}_{i}(k)$ is the significance bin of the loudest candidate of the $k$ th band and $S_{h}\left(f_{k}\right)$ the power spectral density of the data (measured in $1 / \sqrt{\mathrm{Hz}}$ ). The values of the sensitivity depths range between $\mathcal{D}_{\mathrm{CR}_{6}}^{90 \%} \simeq 33(1 / \sqrt{\mathrm{Hz}})$ and $\mathcal{D}_{\mathrm{CR}_{0}}^{90 \%} \simeq 37(1 / \sqrt{\mathrm{Hz}})$. The uncertainties on the upper limit values introduced by this procedure are $\simeq 10 \%$ of the nominal upper limit value. We represent this uncertainty as a shaded region around the upper limit values in Fig. 9. The upper limit values are also provided in tabular form in the

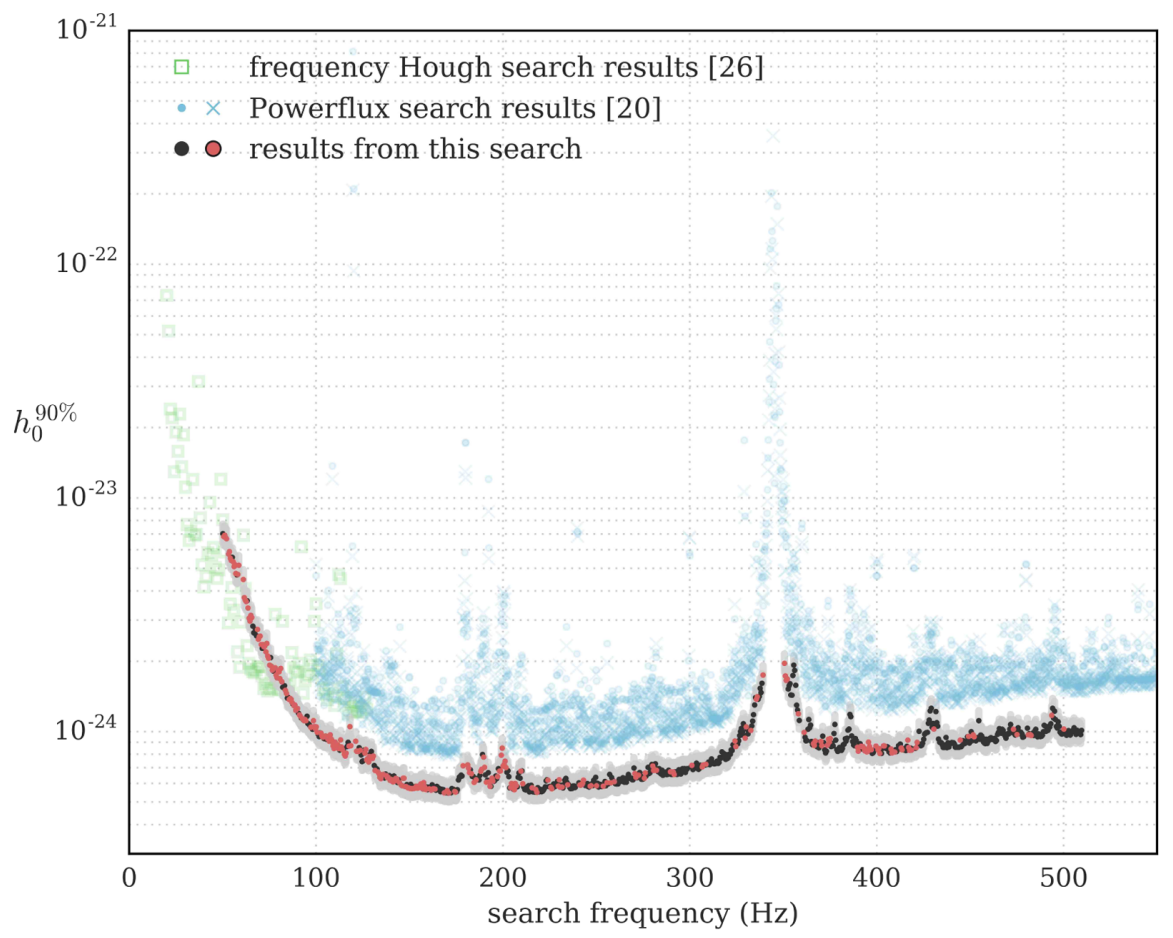

FIG. 9. 90\% confidence upper limits on the gravitational wave amplitude of signals with frequency within half-Hz bands, from the entire sky and within the spin-down range of the search. The light red markers denote half-Hz bands where the upper limit value does not hold for all frequencies in that interval. A list of the excluded frequencies is given in the Appendix. Although not obvious from the figure, due to the quality of the data we were not able to analyze the data in some half-Hz bands, so there are some points missing in the plot. For reference we also plot the upper limit results from two searches: one on the same data (Powerflux) [2] and on contemporary data from the Virgo detector (frequency Hough) [4]. The Powerflux points are obtained by rescaling the best (crosses) and worst-case (dots) upper limit values as explained in the text. It should be noted that the Powerflux upper limits are set at $95 \%$ rather than $90 \%$ but refer to $0.25 \mathrm{~Hz}$ bands rather than half-Hz. 
Appendix in Table II. We do not set upper limits in half-Hz bands where the results are entirely produced with fake data inserted by the cleaning procedure described in Sec. II. Upper limits for such bands will not appear in Table II nor in Fig. 9. There also exist $50 \mathrm{mHz}$ bands that include contributions from fake data as a result of the cleaning procedure or that have been excluded from the analysis because they were marked as disturbed by the visual inspection procedure described in Sec. III B. We mark the half- $\mathrm{Hz}$ bands which host these $50 \mathrm{mHz}$ bands with a different colour (light red) in Fig. 9. In Table IV in the Appendix we provide a complete list of such $50-\mathrm{mHz}$ bands because the upper limit values do not apply to those $50-\mathrm{mHz}$ bands. Finally we note that, due to the cleaning procedure, there exist signal frequency bands where the search results might have contributions from fake data. We list these signal-frequency ranges in Table V. For completeness this table also contains the cleaned bands of Table IV, under the column header "all fake data".

\section{CONCLUSIONS}

Our upper limits are the tightest ever placed for this set of target signals. The smallest value of the GW amplitude upper limit is $5.5 \times 10^{-25}$ in the band $170.5-171 \mathrm{~Hz}$. Figure 9 shows the upper limit values as a function of search frequency. We also show the upper limits from [2], another all-sky search on S6 data, rescaled according to [23] to enable a direct comparison with ours. Under the assumption that the sources are uniformly distributed in space, our search probes a volume in space a few times larger than that of [2]. It should however be noted that [2] examines a much broader parameter space than the one presented here. The Virgo VSR2 and VSR4 science runs were contemporary to the S6 run and more sensitive at low frequency with respect to LIGO. The Virgo data were analyzed in search of continuous signals from the whole sky in the frequency range $20-128 \mathrm{~Hz}$ and a narrower spindown range than that covered here, with $|\dot{f}| \leq 10^{-10} \mathrm{~Hz} / \mathrm{s}$ [4]. Our sensitivity is comparable to that achieved by that search and improves on it above $80 \mathrm{~Hz}$.

Following [24], we define the fraction $x$ of the spin-down rotational energy emitted in gravitational waves. The star's ellipticity necessary to sustain such emission is

$$
\epsilon(f, x \dot{f})=\sqrt{\frac{5 c^{5}}{32 \pi^{4} G} \frac{x \dot{f}}{I f^{5}}},
$$

where $c$ is the speed of light, $G$ is the gravitational constant, $f$ is the GW frequency and $I$ the principal moment of inertia of the star. Correspondingly, $x \dot{f}$ is the spin-down rate that accounts for the emission of GWs, and this is why we refer to it as the GW spin-down. The gravitational wave amplitude $h_{0}$ at the detector coming from a GW source like that of Eq. (14), at a distance $D$ from Earth is

$$
h_{0}(f, \dot{x}, D)=\frac{1}{D} \sqrt{\frac{5 G I}{2 c^{3}} \frac{x \dot{f}}{f}} .
$$

Based on this last equation, we can use our GW amplitude upper limits to bound the minimum distance for compact objects emitting continuous gravitational waves under different assumptions on the object's ellipticity (i.e.

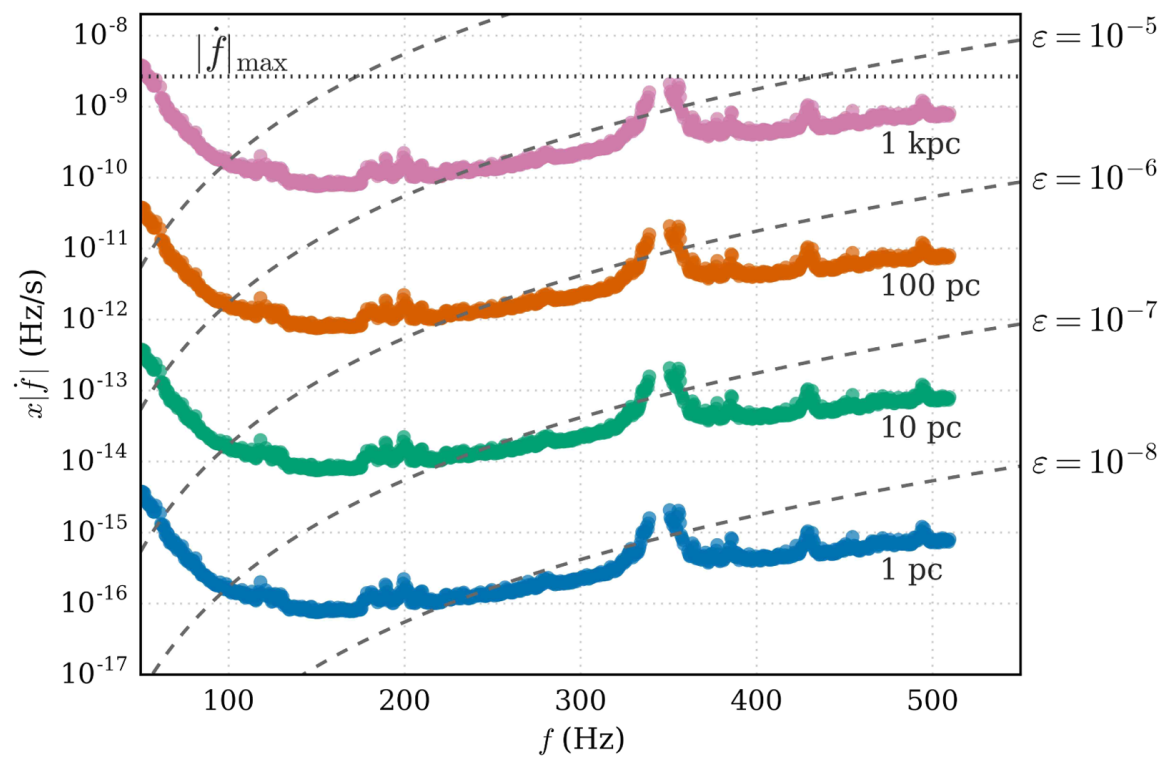

FIG. 10. Gravitational wave amplitude upper limits recast as curves in the $f-x|\dot{f}|$ plane for sources at given distances and having assumed $I=10^{38} \mathrm{~kg} \mathrm{~m}^{2} . f$ is the signal frequency and $x|\dot{f}|$ is the gravitational-wave spin-down, i.e. the fraction of the actual spin-down that accounts for the rotational energy loss due to GW emission. Superimposed are curves of constant ellipticity $\epsilon\left(f, \dot{f} \mid I=10^{38} \mathrm{~kg} \mathrm{~m}^{2}\right)$. The dotted line at $|\dot{f}|_{\max }$ indicates the maximum magnitude of searched spin-down. 
gravitational wave spin-down). This is shown in Fig. 10. We find that for most frequencies above $230 \mathrm{~Hz}$ our upper limits exclude compact objects with ellipticities of $10^{-6} \sqrt{\frac{10^{38} \mathrm{~kg} \mathrm{~m}^{2}}{I}}$ (corresponding to GW spin-downs between $10^{-12} \mathrm{~Hz} / \mathrm{s}$ and $10^{-11} \mathrm{~Hz} / \mathrm{s}$ ) within $100 \mathrm{pc}$ of Earth. Both the ellipticity and the distance ranges span absolutely plausible values and could not have been excluded with other measurements.

We expect the methodology used in this search to serve as a template for the assessment of Einstein@Home run results in the future, for example the next Einstein@ Home run, using advanced LIGO data that is being processed as this paper is written. Results of searches for continuous wave signals could also be mined further, probing subthreshold candidates with a hierarchical series of follow-up searches. This is not the topic of this paper and might be pursued in a forthcoming publication.

\section{ACKNOWLEDGMENTS}

The authors gratefully acknowledge the support of the Einstein@Home volunteers, of the United States National Science Foundation for the construction and operation of the LIGO Laboratory, the Science and Technology Facilities Council of the United Kingdom, the Max-Planck-Society, and the State of Niedersachsen/ Germany for support of the construction and operation of the GEO600 detector, and the Italian Istituto Nazionale di Fisica Nucleare and the French Centre National de la Recherche Scientifique for the construction and operation of the Virgo detector. The authors also gratefully acknowledge the support of the research by these agencies and by the Australian Research Council, the International Science Linkages program of the Commonwealth of Australia, the Council of Scientific and Industrial Research of India, the Istituto Nazionale di Fisica Nucleare of Italy, the Spanish Ministerio de Educación y Ciencia, the Conselleria d'Economia Hisenda i Innovació of the Govern de les Illes Balears, the Foundation for Fundamental Research on Matter supported by the Netherlands Organization for Scientific Research, the Polish Ministry of Science and Higher Education, the FOCUS Programme of Foundation for Polish Science, the Royal Society, the Scottish Funding Council, the Scottish Universities Physics Alliance, The National Aeronautics and Space Administration, the Carnegie Trust, the Leverhulme Trust, the David and Lucile Packard Foundation, the Research Corporation, and the Alfred P. Sloan Foundation.

This document has been assigned LIGO Laboratory document No. LIGO-P1600156-v22.

\section{APPENDIX: TABULAR DATA}

\section{Upper limit values}

TABLE II. First frequency of each half Hz signal frequency band in which we set upper limits and upper limit value for that band.

\begin{tabular}{lc|cc|cc|cc}
\hline \hline$f($ in Hz) & $h_{0}^{90 \%} \times 10^{25}$ & $f$ (in Hz) & $h_{0}^{90 \%} \times 10^{25}$ & $f($ in Hz) & $h_{0}^{90 \%} \times 10^{25}$ & $f($ in Hz) & $h_{0}^{90 \%} \times 10^{25}$ \\
\hline 50.063 & $70.3 \pm 12.8$ & 50.563 & $68.4 \pm 12.5$ & 51.063 & $69.3 \pm 12.6$ & 51.563 & $67.5 \pm 12.4$ \\
52.063 & $66.9 \pm 12.2$ & 53.063 & $57.6 \pm 10.5$ & 53.563 & $58.9 \pm 10.9$ & 54.063 & $55.3 \pm 10.1$ \\
54.563 & $54.0 \pm 9.9$ & 55.063 & $55.7 \pm 10.2$ & 55.563 & $53.3 \pm 9.8$ & 56.063 & $50.9 \pm 9.3$ \\
56.563 & $51.8 \pm 9.5$ & 57.063 & $47.5 \pm 8.7$ & 57.563 & $46.9 \pm 8.6$ & 58.063 & $47.1 \pm 8.6$ \\
58.563 & $51.5 \pm 9.4$ & 61.063 & $44.8 \pm 8.2$ & 61.563 & $37.4 \pm 6.9$ & 62.063 & $36.5 \pm 6.7$ \\
62.563 & $36.0 \pm 6.6$ & 63.063 & $36.3 \pm 6.6$ & 63.563 & $33.8 \pm 6.2$ & 64.063 & $30.6 \pm 5.6$ \\
64.563 & $29.8 \pm 5.4$ & 65.063 & $31.5 \pm 5.9$ & 65.563 & $30.8 \pm 5.7$ & 66.063 & $28.3 \pm 5.2$ \\
66.563 & $26.5 \pm 4.8$ & 67.063 & $26.5 \pm 4.9$ & 67.563 & $27.3 \pm 5.0$ & 68.063 & $25.7 \pm 4.7$ \\
68.563 & $27.4 \pm 5.0$ & 69.063 & $24.8 \pm 4.5$ & 69.563 & $25.5 \pm 4.7$ & 70.063 & $25.7 \pm 4.7$ \\
70.563 & $23.6 \pm 4.3$ & 71.063 & $22.8 \pm 4.2$ & 71.563 & $23.6 \pm 4.3$ & 72.063 & $23.1 \pm 4.2$ \\
72.563 & $23.3 \pm 4.2$ & 73.063 & $22.0 \pm 4.0$ & 73.563 & $23.9 \pm 4.5$ & 74.063 & $21.1 \pm 3.8$ \\
74.563 & $20.6 \pm 3.8$ & 75.063 & $19.3 \pm 3.5$ & 75.563 & $20.8 \pm 3.8$ & 76.063 & $19.0 \pm 3.5$ \\
76.563 & $18.3 \pm 3.4$ & 77.063 & $18.1 \pm 3.3$ & 77.563 & $18.5 \pm 3.4$ & 78.063 & $18.8 \pm 3.4$ \\
78.563 & $17.4 \pm 3.2$ & 79.063 & $17.0 \pm 3.1$ & 79.563 & $18.1 \pm 3.3$ & 80.063 & $18.0 \pm 3.3$ \\
80.563 & $16.9 \pm 3.1$ & 81.063 & $18.7 \pm 3.4$ & 81.563 & $16.3 \pm 3.0$ & 82.063 & $15.5 \pm 2.8$ \\
82.563 & $15.4 \pm 2.8$ & 83.063 & $15.7 \pm 2.9$ & 83.563 & $15.0 \pm 2.8$ & 84.063 & 86.063 \\
84.563 & $13.9 \pm 2.5$ & 85.063 & $14.0 \pm 2.6$ & 85.563 & $13.7 \pm 2.5$ & 83.063 \\
86.563 & $13.8 \pm 2.5$ & 87.063 & $13.3 \pm 2.4$ & 87.563 & $13.1 \pm 2.4$ & 88.063 & $13.9 \pm 2.5$ \\
88.563 & $13.0 \pm 2.4$ & 89.063 & $12.4 \pm 2.3$ & 89.563 & $12.3 \pm 2.3$ & 90.063 & $12.9 \pm 2.4$ \\
\hline
\end{tabular}


TABLE II. (Continued)

\begin{tabular}{|c|c|c|c|c|c|c|c|}
\hline$f($ in $\mathrm{Hz})$ & $h_{0}^{90 \%} \times 10^{25}$ & $f($ in $\mathrm{Hz})$ & $h_{0}^{90 \%} \times 10^{25}$ & $f($ in $\mathrm{Hz})$ & $h_{0}^{90 \%} \times 10^{25}$ & $f($ in $\mathrm{Hz})$ & $h_{0}^{90 \%} \times 10^{25}$ \\
\hline 90.563 & $12.0 \pm 2.2$ & 91.063 & $11.8 \pm 2.2$ & 91.563 & $11.6 \pm 2.1$ & 92.063 & $11.4 \pm 2.1$ \\
\hline 92.563 & $11.3 \pm 2.1$ & 93.063 & $11.2 \pm 2.1$ & 93.563 & $11.1 \pm 2.0$ & 94.063 & $11.3 \pm 2.1$ \\
\hline 94.563 & $11.1 \pm 2.0$ & 95.063 & $11.6 \pm 2.2$ & 95.563 & $10.8 \pm 2.0$ & 96.063 & $10.8 \pm 2.0$ \\
\hline 96.563 & $10.6 \pm 1.9$ & 97.063 & $10.4 \pm 1.9$ & 97.563 & $10.5 \pm 1.9$ & 98.063 & $10.2 \pm 1.9$ \\
\hline 98.563 & $11.1 \pm 2.1$ & 99.063 & $10.5 \pm 1.9$ & 99.563 & $10.3 \pm 1.9$ & 100.063 & $10.5 \pm 1.9$ \\
\hline 100.563 & $9.9 \pm 1.8$ & 101.063 & $9.8 \pm 1.8$ & 101.563 & $9.5 \pm 1.7$ & 102.063 & $9.9 \pm 1.8$ \\
\hline 102.563 & $9.9 \pm 1.8$ & 103.063 & $9.6 \pm 1.8$ & 103.563 & $9.5 \pm 1.7$ & 104.063 & $9.4 \pm 1.7$ \\
\hline 104.563 & $9.3 \pm 1.7$ & 105.063 & $9.6 \pm 1.8$ & 105.563 & $9.3 \pm 1.7$ & 106.063 & $9.3 \pm 1.7$ \\
\hline 106.563 & $9.4 \pm 1.7$ & 107.063 & $9.1 \pm 1.7$ & 107.563 & $9.7 \pm 1.8$ & 108.063 & $9.3 \pm 1.7$ \\
\hline 108.563 & $9.0 \pm 1.7$ & 109.063 & $8.7 \pm 1.6$ & 109.563 & $8.5 \pm 1.5$ & 110.063 & $9.0 \pm 1.7$ \\
\hline 110.563 & $8.6 \pm 1.6$ & 111.063 & $8.6 \pm 1.6$ & 111.563 & $8.8 \pm 1.6$ & 112.063 & $8.5 \pm 1.5$ \\
\hline 112.563 & $8.3 \pm 1.5$ & 113.063 & $9.2 \pm 1.7$ & 113.563 & $8.6 \pm 1.6$ & 114.063 & $8.4 \pm 1.5$ \\
\hline 114.563 & $8.4 \pm 1.6$ & 115.063 & $8.0 \pm 1.5$ & 115.563 & $7.9 \pm 1.4$ & 116.063 & $8.1 \pm 1.5$ \\
\hline 116.563 & $8.6 \pm 1.6$ & 117.063 & $9.0 \pm 1.7$ & 117.563 & $8.7 \pm 1.6$ & 118.063 & $10.5 \pm 1.9$ \\
\hline 118.563 & $8.7 \pm 1.6$ & 121.063 & $9.1 \pm 1.7$ & 121.563 & $8.2 \pm 1.5$ & 122.063 & $8.3 \pm 1.5$ \\
\hline 122.563 & $8.2 \pm 1.5$ & 123.063 & $8.5 \pm 1.6$ & 123.563 & $8.3 \pm 1.5$ & 124.063 & $8.0 \pm 1.4$ \\
\hline 124.563 & $7.4 \pm 1.4$ & 125.063 & $7.5 \pm 1.4$ & 125.563 & $8.3 \pm 1.5$ & 126.063 & $8.1 \pm 1.5$ \\
\hline 126.563 & $8.4 \pm 1.5$ & 127.063 & $7.6 \pm 1.4$ & 127.563 & $7.7 \pm 1.4$ & 128.063 & $7.4 \pm 1.4$ \\
\hline 128.563 & $7.8 \pm 1.4$ & 129.063 & $8.0 \pm 1.5$ & 129.563 & $8.2 \pm 1.5$ & 130.063 & $7.7 \pm 1.4$ \\
\hline 130.563 & $7.9 \pm 1.4$ & 131.063 & $7.2 \pm 1.3$ & 131.563 & $6.8 \pm 1.2$ & 132.063 & $7.0 \pm 1.3$ \\
\hline 132.563 & $6.9 \pm 1.3$ & 133.063 & $6.7 \pm 1.2$ & 133.563 & $6.6 \pm 1.2$ & 134.063 & $6.4 \pm 1.2$ \\
\hline 134.563 & $6.3 \pm 1.2$ & 135.063 & $6.5 \pm 1.2$ & 135.563 & $6.5 \pm 1.2$ & 136.063 & $6.6 \pm 1.2$ \\
\hline 136.563 & $6.3 \pm 1.2$ & 137.063 & $6.6 \pm 1.2$ & 137.563 & $6.5 \pm 1.2$ & 138.063 & $6.4 \pm 1.2$ \\
\hline 138.563 & $6.4 \pm 1.2$ & 139.063 & $6.5 \pm 1.2$ & 139.563 & $6.2 \pm 1.1$ & 140.063 & $6.3 \pm 1.1$ \\
\hline 140.563 & $6.2 \pm 1.1$ & 141.063 & $6.1 \pm 1.1$ & 141.563 & $6.5 \pm 1.2$ & 142.063 & $6.2 \pm 1.1$ \\
\hline 142.563 & $6.3 \pm 1.2$ & 143.063 & $6.3 \pm 1.1$ & 143.563 & $6.0 \pm 1.1$ & 144.063 & $6.2 \pm 1.1$ \\
\hline 144.563 & $6.0 \pm 1.1$ & 145.563 & $5.9 \pm 1.1$ & 146.063 & $5.9 \pm 1.1$ & 146.563 & $6.3 \pm 1.2$ \\
\hline 147.063 & $6.3 \pm 1.2$ & 147.563 & $5.8 \pm 1.1$ & 148.063 & $5.8 \pm 1.1$ & 148.563 & $5.9 \pm 1.1$ \\
\hline 149.063 & $5.8 \pm 1.1$ & 149.563 & $5.7 \pm 1.0$ & 150.063 & $5.7 \pm 1.0$ & 150.563 & $6.0 \pm 1.1$ \\
\hline 151.063 & $5.7 \pm 1.0$ & 151.563 & $5.7 \pm 1.0$ & 152.063 & $5.7 \pm 1.1$ & 152.563 & $5.7 \pm 1.0$ \\
\hline 153.063 & $5.8 \pm 1.1$ & 153.563 & $5.8 \pm 1.1$ & 154.063 & $5.7 \pm 1.0$ & 154.563 & $5.7 \pm 1.1$ \\
\hline 155.063 & $5.9 \pm 1.1$ & 155.563 & $5.9 \pm 1.1$ & 156.063 & $6.0 \pm 1.1$ & 156.563 & $6.0 \pm 1.1$ \\
\hline 157.063 & $5.7 \pm 1.0$ & 157.563 & $6.0 \pm 1.1$ & 158.063 & $5.8 \pm 1.1$ & 158.563 & $5.7 \pm 1.0$ \\
\hline 159.063 & $5.8 \pm 1.1$ & 159.563 & $5.6 \pm 1.0$ & 160.063 & $5.8 \pm 1.1$ & 160.563 & $5.7 \pm 1.0$ \\
\hline 161.063 & $5.7 \pm 1.0$ & 161.563 & $5.6 \pm 1.0$ & 162.063 & $5.9 \pm 1.1$ & 162.563 & $5.7 \pm 1.0$ \\
\hline 163.063 & $5.7 \pm 1.0$ & 163.563 & $5.7 \pm 1.0$ & 164.063 & $5.6 \pm 1.0$ & 164.563 & $5.8 \pm 1.1$ \\
\hline 165.063 & $5.7 \pm 1.0$ & 165.563 & $5.7 \pm 1.0$ & 166.063 & $5.7 \pm 1.0$ & 166.563 & $5.5 \pm 1.0$ \\
\hline 167.063 & $5.7 \pm 1.0$ & 167.563 & $5.6 \pm 1.0$ & 168.063 & $5.6 \pm 1.0$ & 168.563 & $5.5 \pm 1.0$ \\
\hline 169.063 & $5.5 \pm 1.0$ & 169.563 & $5.5 \pm 1.0$ & 170.063 & $5.6 \pm 1.0$ & 170.563 & $5.5 \pm 1.0$ \\
\hline 171.063 & $5.5 \pm 1.0$ & 171.563 & $5.5 \pm 1.0$ & 172.063 & $5.5 \pm 1.0$ & 172.563 & $5.7 \pm 1.0$ \\
\hline 173.063 & $5.6 \pm 1.0$ & 173.563 & $5.7 \pm 1.0$ & 174.063 & $5.5 \pm 1.0$ & 174.563 & $5.5 \pm 1.0$ \\
\hline 175.063 & $5.5 \pm 1.0$ & 175.563 & $5.6 \pm 1.0$ & 176.063 & $6.2 \pm 1.1$ & 176.563 & $6.4 \pm 1.2$ \\
\hline 177.063 & $6.4 \pm 1.2$ & 177.563 & $6.5 \pm 1.2$ & 178.063 & $6.5 \pm 1.2$ & 178.563 & $7.2 \pm 1.3$ \\
\hline 181.063 & $7.2 \pm 1.3$ & 181.563 & $7.0 \pm 1.3$ & 182.063 & $6.7 \pm 1.2$ & 182.563 & $6.9 \pm 1.3$ \\
\hline 183.063 & $6.6 \pm 1.2$ & 183.563 & $6.4 \pm 1.2$ & 184.063 & $6.4 \pm 1.2$ & 184.563 & $6.1 \pm 1.1$ \\
\hline 185.063 & $6.3 \pm 1.2$ & 185.563 & $6.2 \pm 1.1$ & 186.063 & $6.2 \pm 1.1$ & 186.563 & $6.3 \pm 1.2$ \\
\hline 187.063 & $6.2 \pm 1.1$ & 187.563 & $6.5 \pm 1.2$ & 188.063 & $6.8 \pm 1.2$ & 188.563 & $6.9 \pm 1.3$ \\
\hline 189.063 & $8.0 \pm 1.5$ & 189.563 & $7.8 \pm 1.4$ & 190.063 & $7.0 \pm 1.3$ & 190.563 & $6.5 \pm 1.2$ \\
\hline 191.063 & $6.1 \pm 1.1$ & 191.563 & $6.2 \pm 1.1$ & 192.063 & $6.7 \pm 1.3$ & 192.563 & $6.1 \pm 1.1$ \\
\hline 193.063 & $5.8 \pm 1.1$ & 193.563 & $5.8 \pm 1.1$ & 194.063 & $6.3 \pm 1.2$ & 194.563 & $6.1 \pm 1.1$ \\
\hline 195.063 & $6.1 \pm 1.1$ & 195.563 & $6.2 \pm 1.1$ & 196.063 & $6.5 \pm 1.2$ & 196.563 & $6.3 \pm 1.2$ \\
\hline 197.063 & $6.4 \pm 1.2$ & 197.563 & $6.9 \pm 1.3$ & 198.063 & $6.8 \pm 1.2$ & 198.563 & $6.8 \pm 1.2$ \\
\hline 199.063 & $7.9 \pm 1.4$ & 199.563 & $8.5 \pm 1.6$ & 200.063 & $7.1 \pm 1.3$ & 200.563 & $7.3 \pm 1.3$ \\
\hline 201.063 & $7.5 \pm 1.4$ & 201.563 & $7.0 \pm 1.3$ & 202.063 & $6.7 \pm 1.2$ & 202.563 & $6.8 \pm 1.2$ \\
\hline 203.063 & $6.4 \pm 1.2$ & 203.563 & $5.7 \pm 1.1$ & 204.063 & $5.8 \pm 1.1$ & 204.563 & $6.0 \pm 1.1$ \\
\hline
\end{tabular}


TABLE II. (Continued)

\begin{tabular}{|c|c|c|c|c|c|c|c|}
\hline$f($ in $\mathrm{Hz})$ & $h_{0}^{90 \%} \times 10^{25}$ & $f($ in $\mathrm{Hz})$ & $h_{0}^{90 \%} \times 10^{25}$ & $f($ in $\mathrm{Hz})$ & $h_{0}^{90 \%} \times 10^{25}$ & $f($ in $\mathrm{Hz})$ & $h_{0}^{90 \%} \times 10^{25}$ \\
\hline 205.063 & $5.8 \pm 1.1$ & 205.563 & $5.7 \pm 1.0$ & 206.063 & $5.6 \pm 1.0$ & 206.563 & $6.0 \pm 1.1$ \\
\hline 207.063 & $5.9 \pm 1.1$ & 207.563 & $5.8 \pm 1.1$ & 208.063 & $6.4 \pm 1.2$ & 208.563 & $6.7 \pm 1.2$ \\
\hline 209.063 & $6.3 \pm 1.2$ & 209.563 & $6.8 \pm 1.2$ & 210.063 & $6.8 \pm 1.2$ & 210.563 & $6.0 \pm 1.1$ \\
\hline 211.063 & $5.8 \pm 1.1$ & 211.563 & $5.7 \pm 1.0$ & 212.063 & $5.6 \pm 1.0$ & 212.563 & $5.8 \pm 1.1$ \\
\hline 213.063 & $5.7 \pm 1.0$ & 213.563 & $5.9 \pm 1.1$ & 214.063 & $5.5 \pm 1.0$ & 214.563 & $5.8 \pm 1.1$ \\
\hline 215.063 & $5.9 \pm 1.1$ & 215.563 & $5.8 \pm 1.1$ & 216.063 & $5.5 \pm 1.0$ & 216.563 & $5.5 \pm 1.0$ \\
\hline 217.063 & $5.5 \pm 1.0$ & 217.563 & $5.7 \pm 1.0$ & 218.063 & $5.5 \pm 1.0$ & 218.563 & $5.8 \pm 1.1$ \\
\hline 219.063 & $5.5 \pm 1.0$ & 219.563 & $5.7 \pm 1.0$ & 220.063 & $5.7 \pm 1.0$ & 220.563 & $5.5 \pm 1.0$ \\
\hline 221.063 & $5.6 \pm 1.0$ & 221.563 & $5.6 \pm 1.0$ & 222.063 & $5.7 \pm 1.0$ & 222.563 & $5.8 \pm 1.1$ \\
\hline 223.063 & $6.2 \pm 1.1$ & 223.563 & $6.2 \pm 1.1$ & 224.063 & $6.2 \pm 1.1$ & 224.563 & $5.8 \pm 1.1$ \\
\hline 225.063 & $5.8 \pm 1.1$ & 225.563 & $5.8 \pm 1.1$ & 226.063 & $5.7 \pm 1.0$ & 226.563 & $5.7 \pm 1.0$ \\
\hline 227.063 & $6.0 \pm 1.1$ & 227.563 & $5.8 \pm 1.1$ & 228.063 & $5.9 \pm 1.1$ & 228.563 & $5.9 \pm 1.1$ \\
\hline 229.063 & $6.1 \pm 1.1$ & 229.563 & $5.9 \pm 1.1$ & 230.063 & $6.2 \pm 1.1$ & 230.563 & $5.8 \pm 1.1$ \\
\hline 231.063 & $5.9 \pm 1.1$ & 231.563 & $5.8 \pm 1.1$ & 232.063 & $5.7 \pm 1.1$ & 232.563 & $5.9 \pm 1.1$ \\
\hline 233.063 & $6.2 \pm 1.1$ & 233.563 & $6.3 \pm 1.1$ & 234.063 & $6.1 \pm 1.1$ & 234.563 & $5.9 \pm 1.1$ \\
\hline 235.063 & $5.9 \pm 1.1$ & 235.563 & $5.8 \pm 1.1$ & 236.063 & $5.7 \pm 1.0$ & 236.563 & $5.7 \pm 1.0$ \\
\hline 237.063 & $5.7 \pm 1.0$ & 237.563 & $5.9 \pm 1.1$ & 238.063 & $5.9 \pm 1.1$ & 238.563 & $5.8 \pm 1.1$ \\
\hline 240.563 & $6.0 \pm 1.1$ & 241.063 & $5.9 \pm 1.1$ & 241.563 & $5.9 \pm 1.1$ & 242.063 & $5.9 \pm 1.1$ \\
\hline 242.563 & $6.0 \pm 1.1$ & 243.063 & $6.2 \pm 1.1$ & 243.563 & $6.0 \pm 1.1$ & 244.063 & $5.9 \pm 1.1$ \\
\hline 244.563 & $5.9 \pm 1.1$ & 245.063 & $6.0 \pm 1.1$ & 245.563 & $5.8 \pm 1.1$ & 246.063 & $5.8 \pm 1.1$ \\
\hline 246.563 & $5.8 \pm 1.1$ & 247.063 & $5.9 \pm 1.1$ & 247.563 & $6.0 \pm 1.1$ & 248.063 & $5.9 \pm 1.1$ \\
\hline 248.563 & $6.2 \pm 1.1$ & 249.063 & $6.1 \pm 1.1$ & 249.563 & $6.4 \pm 1.2$ & 250.063 & $5.9 \pm 1.1$ \\
\hline 250.563 & $6.0 \pm 1.1$ & 251.063 & $5.8 \pm 1.1$ & 251.563 & $5.9 \pm 1.1$ & 252.063 & $5.9 \pm 1.1$ \\
\hline 252.563 & $5.8 \pm 1.1$ & 253.063 & $5.8 \pm 1.1$ & 253.563 & $5.8 \pm 1.1$ & 254.063 & $5.9 \pm 1.1$ \\
\hline 254.563 & $6.1 \pm 1.1$ & 255.063 & $5.9 \pm 1.1$ & 255.563 & $6.1 \pm 1.1$ & 256.063 & $6.0 \pm 1.1$ \\
\hline 256.563 & $6.0 \pm 1.1$ & 257.063 & $6.6 \pm 1.2$ & 257.563 & $6.0 \pm 1.1$ & 258.063 & $6.4 \pm 1.2$ \\
\hline 258.563 & $6.2 \pm 1.1$ & 259.063 & $6.1 \pm 1.1$ & 259.563 & $6.1 \pm 1.1$ & 260.063 & $6.0 \pm 1.1$ \\
\hline 260.563 & $6.0 \pm 1.1$ & 261.063 & $6.0 \pm 1.1$ & 261.563 & $6.0 \pm 1.1$ & 262.063 & $6.3 \pm 1.1$ \\
\hline 262.563 & $6.1 \pm 1.1$ & 263.063 & $6.2 \pm 1.1$ & 263.563 & $6.2 \pm 1.1$ & 264.063 & $6.3 \pm 1.2$ \\
\hline 264.563 & $6.1 \pm 1.1$ & 265.063 & $6.1 \pm 1.1$ & 265.563 & $6.3 \pm 1.1$ & 266.063 & $6.1 \pm 1.1$ \\
\hline 266.563 & $6.4 \pm 1.2$ & 267.063 & $6.6 \pm 1.2$ & 267.563 & $6.3 \pm 1.2$ & 268.063 & $6.4 \pm 1.2$ \\
\hline 268.563 & $6.3 \pm 1.2$ & 269.063 & $6.2 \pm 1.1$ & 269.563 & $6.2 \pm 1.1$ & 270.063 & $7.0 \pm 1.3$ \\
\hline 270.563 & $6.6 \pm 1.2$ & 271.063 & $6.4 \pm 1.2$ & 271.563 & $6.3 \pm 1.2$ & 272.063 & $6.6 \pm 1.2$ \\
\hline 272.563 & $6.5 \pm 1.2$ & 273.063 & $6.7 \pm 1.2$ & 273.563 & $6.5 \pm 1.2$ & 274.063 & $6.2 \pm 1.1$ \\
\hline 274.563 & $6.3 \pm 1.1$ & 275.063 & $6.3 \pm 1.1$ & 275.563 & $6.3 \pm 1.2$ & 276.063 & $6.7 \pm 1.2$ \\
\hline 276.563 & $6.5 \pm 1.2$ & 277.063 & $6.6 \pm 1.2$ & 277.563 & $7.0 \pm 1.3$ & 278.063 & $6.6 \pm 1.2$ \\
\hline 278.563 & $6.7 \pm 1.2$ & 279.063 & $6.8 \pm 1.3$ & 279.563 & $7.2 \pm 1.3$ & 280.063 & $7.1 \pm 1.3$ \\
\hline 280.563 & $6.8 \pm 1.2$ & 281.063 & $6.9 \pm 1.3$ & 281.563 & $7.3 \pm 1.3$ & 282.063 & $6.8 \pm 1.3$ \\
\hline 282.563 & $6.9 \pm 1.3$ & 283.063 & $6.7 \pm 1.2$ & 283.563 & $6.9 \pm 1.3$ & 284.063 & $6.6 \pm 1.2$ \\
\hline 284.563 & $6.6 \pm 1.2$ & 285.063 & $6.8 \pm 1.3$ & 285.563 & $6.5 \pm 1.2$ & 286.063 & $6.7 \pm 1.2$ \\
\hline 286.563 & $6.6 \pm 1.2$ & 287.063 & $6.7 \pm 1.2$ & 287.563 & $6.5 \pm 1.2$ & 288.063 & $6.6 \pm 1.2$ \\
\hline 288.563 & $6.8 \pm 1.2$ & 289.063 & $6.6 \pm 1.2$ & 289.563 & $6.7 \pm 1.2$ & 290.063 & $6.6 \pm 1.2$ \\
\hline 290.563 & $6.6 \pm 1.2$ & 291.063 & $6.7 \pm 1.2$ & 291.563 & $6.6 \pm 1.2$ & 292.063 & $6.7 \pm 1.2$ \\
\hline 292.563 & $6.6 \pm 1.2$ & 293.063 & $6.6 \pm 1.2$ & 293.563 & $6.8 \pm 1.2$ & 294.063 & $6.9 \pm 1.3$ \\
\hline 294.563 & $6.6 \pm 1.2$ & 295.063 & $6.6 \pm 1.2$ & 295.563 & $6.9 \pm 1.3$ & 296.063 & $6.9 \pm 1.3$ \\
\hline 296.563 & $6.7 \pm 1.2$ & 297.063 & $6.9 \pm 1.3$ & 297.563 & $6.7 \pm 1.2$ & 298.063 & $6.9 \pm 1.3$ \\
\hline 298.563 & $6.9 \pm 1.3$ & 300.563 & $7.1 \pm 1.3$ & 301.063 & $7.2 \pm 1.3$ & 301.563 & $6.9 \pm 1.3$ \\
\hline 302.063 & $6.9 \pm 1.3$ & 302.563 & $7.1 \pm 1.3$ & 303.063 & $7.1 \pm 1.3$ & 303.563 & $7.3 \pm 1.3$ \\
\hline 304.063 & $7.2 \pm 1.3$ & 304.563 & $6.9 \pm 1.3$ & 305.063 & $7.0 \pm 1.3$ & 305.563 & $7.2 \pm 1.3$ \\
\hline 306.063 & $7.1 \pm 1.3$ & 306.563 & $7.1 \pm 1.3$ & 307.063 & $7.2 \pm 1.3$ & 307.563 & $7.2 \pm 1.3$ \\
\hline 308.063 & $7.2 \pm 1.3$ & 308.563 & $7.3 \pm 1.3$ & 309.063 & $7.2 \pm 1.3$ & 309.563 & $7.3 \pm 1.3$ \\
\hline 310.063 & $7.4 \pm 1.4$ & 310.563 & $7.2 \pm 1.3$ & 311.063 & $7.5 \pm 1.4$ & 311.563 & $7.6 \pm 1.4$ \\
\hline 312.063 & $7.4 \pm 1.4$ & 312.563 & $7.3 \pm 1.3$ & 313.063 & $7.3 \pm 1.3$ & 313.563 & $7.3 \pm 1.3$ \\
\hline 314.063 & $7.3 \pm 1.3$ & 314.563 & $7.5 \pm 1.4$ & 315.063 & $7.3 \pm 1.3$ & 315.563 & $7.4 \pm 1.4$ \\
\hline 316.063 & $7.8 \pm 1.4$ & 316.563 & $7.7 \pm 1.4$ & 317.063 & $8.2 \pm 1.5$ & 317.563 & $7.8 \pm 1.4$ \\
\hline
\end{tabular}


TABLE II. (Continued)

\begin{tabular}{|c|c|c|c|c|c|c|c|}
\hline$f($ in $\mathrm{Hz})$ & $h_{0}^{90 \%} \times 10^{25}$ & $f($ in $\mathrm{Hz})$ & $h_{0}^{90 \%} \times 10^{25}$ & $f($ in $\mathrm{Hz})$ & $h_{0}^{90 \%} \times 10^{25}$ & $f($ in $\mathrm{Hz})$ & $h_{0}^{90 \%} \times 10^{25}$ \\
\hline 318.063 & $8.1 \pm 1.5$ & 318.563 & $7.8 \pm 1.4$ & 319.063 & $7.6 \pm 1.4$ & 319.563 & $8.0 \pm 1.5$ \\
\hline 320.063 & $7.7 \pm 1.4$ & 320.563 & $7.7 \pm 1.4$ & 321.063 & $8.0 \pm 1.5$ & 321.563 & $8.0 \pm 1.5$ \\
\hline 322.063 & $8.4 \pm 1.5$ & 322.563 & $8.3 \pm 1.5$ & 323.063 & $8.8 \pm 1.6$ & 323.563 & $8.7 \pm 1.6$ \\
\hline 324.063 & $9.1 \pm 1.7$ & 324.563 & $8.6 \pm 1.6$ & 325.063 & $9.0 \pm 1.6$ & 325.563 & $8.9 \pm 1.6$ \\
\hline 326.063 & $9.1 \pm 1.7$ & 326.563 & $9.6 \pm 1.8$ & 327.063 & $10.0 \pm 1.8$ & 327.563 & $10.3 \pm 1.9$ \\
\hline 328.063 & $10.0 \pm 1.8$ & 328.563 & $10.8 \pm 2.0$ & 329.063 & $9.6 \pm 1.8$ & 329.563 & $9.4 \pm 1.7$ \\
\hline 330.063 & $10.5 \pm 1.9$ & 330.563 & $10.2 \pm 1.9$ & 331.063 & $9.8 \pm 1.8$ & 331.563 & $10.2 \pm 1.9$ \\
\hline 332.063 & $10.2 \pm 1.9$ & 332.563 & $10.1 \pm 1.9$ & 333.063 & $10.6 \pm 1.9$ & 333.563 & $11.1 \pm 2.0$ \\
\hline 334.063 & $11.8 \pm 2.2$ & 334.563 & $13.0 \pm 2.4$ & 335.063 & $14.0 \pm 2.6$ & 335.563 & $13.8 \pm 2.5$ \\
\hline 336.063 & $14.1 \pm 2.6$ & 336.563 & $13.8 \pm 2.5$ & 337.063 & $14.1 \pm 2.6$ & 337.563 & $15.3 \pm 2.9$ \\
\hline 338.063 & $14.7 \pm 2.7$ & 338.563 & $16.1 \pm 2.9$ & 339.063 & $17.4 \pm 3.2$ & 350.563 & $19.6 \pm 3.6$ \\
\hline 351.063 & $17.3 \pm 3.2$ & 351.563 & $16.6 \pm 3.0$ & 352.063 & $16.8 \pm 3.1$ & 352.563 & $15.2 \pm 2.8$ \\
\hline 353.063 & $15.7 \pm 2.9$ & 353.563 & $16.0 \pm 2.9$ & 354.063 & $14.4 \pm 2.6$ & 354.563 & $14.3 \pm 2.6$ \\
\hline 355.063 & $17.4 \pm 3.2$ & 355.563 & $19.3 \pm 3.5$ & 356.063 & $18.3 \pm 3.3$ & 356.563 & $15.8 \pm 2.9$ \\
\hline 357.063 & $13.0 \pm 2.4$ & 357.563 & $12.4 \pm 2.3$ & 358.063 & $11.9 \pm 2.2$ & 358.563 & $11.2 \pm 2.0$ \\
\hline 361.063 & $9.9 \pm 1.8$ & 361.563 & $9.5 \pm 1.7$ & 362.063 & $9.1 \pm 1.7$ & 362.563 & $9.2 \pm 1.7$ \\
\hline 363.063 & $10.2 \pm 1.9$ & 363.563 & $11.3 \pm 2.1$ & 364.063 & $10.4 \pm 1.9$ & 364.563 & $10.7 \pm 2.0$ \\
\hline 365.063 & $9.1 \pm 1.7$ & 365.563 & $8.8 \pm 1.6$ & 366.063 & $8.9 \pm 1.6$ & 366.563 & $8.7 \pm 1.6$ \\
\hline 367.063 & $9.1 \pm 1.7$ & 367.563 & $9.0 \pm 1.6$ & 368.063 & $8.6 \pm 1.6$ & 368.563 & $9.0 \pm 1.6$ \\
\hline 369.063 & $8.5 \pm 1.6$ & 369.563 & $8.9 \pm 1.6$ & 370.063 & $9.1 \pm 1.7$ & 370.563 & $8.7 \pm 1.6$ \\
\hline 371.063 & $9.6 \pm 1.8$ & 371.563 & $9.0 \pm 1.6$ & 372.063 & $8.4 \pm 1.5$ & 372.563 & $8.1 \pm 1.5$ \\
\hline 373.063 & $8.3 \pm 1.5$ & 373.563 & $8.7 \pm 1.6$ & 374.063 & $9.3 \pm 1.7$ & 374.563 & $9.0 \pm 1.6$ \\
\hline 375.063 & $9.3 \pm 1.7$ & 375.563 & $8.6 \pm 1.6$ & 376.063 & $8.9 \pm 1.6$ & 376.563 & $8.7 \pm 1.6$ \\
\hline 377.063 & $9.8 \pm 1.8$ & 377.563 & $10.7 \pm 2.0$ & 378.063 & $9.2 \pm 1.7$ & 378.563 & $8.4 \pm 1.5$ \\
\hline 379.063 & $8.2 \pm 1.5$ & 379.563 & $8.4 \pm 1.5$ & 380.063 & $8.6 \pm 1.6$ & 380.563 & $8.5 \pm 1.5$ \\
\hline 381.063 & $8.3 \pm 1.5$ & 381.563 & $8.5 \pm 1.6$ & 382.063 & $8.8 \pm 1.6$ & 382.563 & $8.9 \pm 1.6$ \\
\hline 383.063 & $9.3 \pm 1.7$ & 383.563 & $9.1 \pm 1.7$ & 384.063 & $9.4 \pm 1.7$ & 384.563 & $10.2 \pm 1.9$ \\
\hline 385.063 & $10.3 \pm 1.9$ & 385.563 & $11.9 \pm 2.2$ & 386.063 & $11.6 \pm 2.1$ & 386.563 & $9.6 \pm 1.7$ \\
\hline 387.063 & $9.1 \pm 1.7$ & 387.563 & $8.8 \pm 1.6$ & 388.063 & $8.7 \pm 1.6$ & 388.563 & $9.3 \pm 1.7$ \\
\hline 389.063 & $9.2 \pm 1.7$ & 389.563 & $8.6 \pm 1.6$ & 390.063 & $8.3 \pm 1.5$ & 390.563 & $8.8 \pm 1.6$ \\
\hline 391.063 & $8.9 \pm 1.6$ & 391.563 & $8.7 \pm 1.6$ & 392.063 & $8.4 \pm 1.5$ & 392.563 & $8.6 \pm 1.6$ \\
\hline 393.063 & $8.5 \pm 1.6$ & 393.563 & $8.3 \pm 1.5$ & 394.063 & $8.4 \pm 1.5$ & 394.563 & $8.2 \pm 1.5$ \\
\hline 395.063 & $8.2 \pm 1.5$ & 395.563 & $9.0 \pm 1.7$ & 396.063 & $8.6 \pm 1.6$ & 396.563 & $8.4 \pm 1.5$ \\
\hline 397.063 & $8.4 \pm 1.5$ & 397.563 & $8.1 \pm 1.5$ & 398.063 & $8.1 \pm 1.5$ & 398.563 & $8.3 \pm 1.5$ \\
\hline 399.063 & $8.4 \pm 1.5$ & 399.563 & $8.7 \pm 1.6$ & 400.563 & $8.3 \pm 1.5$ & 401.063 & $8.5 \pm 1.6$ \\
\hline 401.563 & $8.1 \pm 1.5$ & 402.063 & $8.1 \pm 1.5$ & 402.563 & $8.1 \pm 1.5$ & 403.063 & $8.5 \pm 1.6$ \\
\hline 403.563 & $8.7 \pm 1.6$ & 404.063 & $8.7 \pm 1.6$ & 404.563 & $8.6 \pm 1.6$ & 405.063 & $8.5 \pm 1.6$ \\
\hline 405.563 & $8.6 \pm 1.6$ & 406.063 & $8.6 \pm 1.6$ & 406.563 & $8.2 \pm 1.5$ & 407.063 & $8.1 \pm 1.5$ \\
\hline 407.563 & $8.4 \pm 1.5$ & 408.063 & $8.1 \pm 1.5$ & 408.563 & $8.2 \pm 1.5$ & 409.063 & $8.2 \pm 1.5$ \\
\hline 409.563 & $8.2 \pm 1.5$ & 410.063 & $8.4 \pm 1.5$ & 410.563 & $8.2 \pm 1.5$ & 411.063 & $8.4 \pm 1.5$ \\
\hline 411.563 & $8.7 \pm 1.6$ & 412.063 & $8.7 \pm 1.6$ & 412.563 & $8.8 \pm 1.6$ & 413.063 & $8.5 \pm 1.6$ \\
\hline 413.563 & $8.4 \pm 1.5$ & 414.063 & $8.4 \pm 1.5$ & 414.563 & $8.5 \pm 1.5$ & 415.063 & $8.5 \pm 1.5$ \\
\hline 415.563 & $8.3 \pm 1.5$ & 416.063 & $8.7 \pm 1.6$ & 416.563 & $9.2 \pm 1.7$ & 417.063 & $8.6 \pm 1.6$ \\
\hline 417.563 & $8.3 \pm 1.5$ & 418.063 & $8.5 \pm 1.6$ & 418.563 & $8.3 \pm 1.5$ & 420.563 & $8.5 \pm 1.6$ \\
\hline 421.063 & $8.5 \pm 1.6$ & 421.563 & $9.0 \pm 1.7$ & 422.063 & $8.8 \pm 1.6$ & 422.563 & $10.0 \pm 1.8$ \\
\hline 423.063 & $8.8 \pm 1.6$ & 423.563 & $8.7 \pm 1.6$ & 424.063 & $8.7 \pm 1.6$ & 424.563 & $8.9 \pm 1.6$ \\
\hline 425.063 & $9.3 \pm 1.7$ & 425.563 & $9.7 \pm 1.8$ & 426.063 & $9.8 \pm 1.8$ & 426.563 & $9.9 \pm 1.8$ \\
\hline 427.063 & $9.9 \pm 1.8$ & 427.563 & $10.9 \pm 2.0$ & 428.063 & $11.2 \pm 2.1$ & 428.563 & $12.3 \pm 2.2$ \\
\hline 429.063 & $12.6 \pm 2.3$ & 429.563 & $10.4 \pm 1.9$ & 430.063 & $10.8 \pm 2.0$ & 430.563 & $10.3 \pm 1.9$ \\
\hline 431.063 & $10.5 \pm 1.9$ & 431.563 & $12.2 \pm 2.2$ & 432.063 & $10.9 \pm 2.0$ & 432.563 & $10.6 \pm 1.9$ \\
\hline 433.063 & $9.3 \pm 1.7$ & 433.563 & $9.0 \pm 1.6$ & 434.063 & $9.0 \pm 1.6$ & 434.563 & $9.0 \pm 1.6$ \\
\hline 435.063 & $8.8 \pm 1.6$ & 435.563 & $8.6 \pm 1.6$ & 436.063 & $8.6 \pm 1.6$ & 436.563 & $8.8 \pm 1.6$ \\
\hline 437.063 & $9.4 \pm 1.8$ & 437.563 & $8.5 \pm 1.6$ & 438.063 & $9.0 \pm 1.6$ & 438.563 & $8.6 \pm 1.6$ \\
\hline 439.063 & $8.8 \pm 1.6$ & 439.563 & $8.9 \pm 1.6$ & 440.063 & $9.0 \pm 1.6$ & 440.563 & $8.9 \pm 1.6$ \\
\hline 441.063 & $9.0 \pm 1.6$ & 441.563 & $8.8 \pm 1.6$ & 442.063 & $8.6 \pm 1.6$ & 442.563 & $8.6 \pm 1.6$ \\
\hline
\end{tabular}


TABLE II. (Continued)

\begin{tabular}{|c|c|c|c|c|c|c|c|}
\hline$f($ in $\mathrm{Hz})$ & $h_{0}^{90 \%} \times 10^{25}$ & $f($ in $\mathrm{Hz})$ & $h_{0}^{90 \%} \times 10^{25}$ & $f($ in $\mathrm{Hz})$ & $h_{0}^{90 \%} \times 10^{25}$ & $f($ in $\mathrm{Hz})$ & $h_{0}^{90 \%} \times 10^{25}$ \\
\hline 443.063 & $8.6 \pm 1.6$ & 443.563 & $8.6 \pm 1.6$ & 444.063 & $8.6 \pm 1.6$ & 444.563 & $9.2 \pm 1.7$ \\
\hline 445.063 & $8.6 \pm 1.6$ & 445.563 & $8.7 \pm 1.6$ & 446.063 & $9.2 \pm 1.7$ & 446.563 & $9.1 \pm 1.7$ \\
\hline 447.063 & $8.9 \pm 1.6$ & 447.563 & $9.0 \pm 1.7$ & 448.063 & $9.0 \pm 1.6$ & 448.563 & $9.5 \pm 1.7$ \\
\hline 449.063 & $9.5 \pm 1.8$ & 449.563 & $8.9 \pm 1.6$ & 450.063 & $9.1 \pm 1.7$ & 450.563 & $10.0 \pm 1.8$ \\
\hline 451.063 & $9.2 \pm 1.7$ & 451.563 & $9.7 \pm 1.8$ & 452.063 & $9.5 \pm 1.7$ & 452.563 & $9.2 \pm 1.7$ \\
\hline 453.063 & $9.1 \pm 1.7$ & 453.563 & $9.4 \pm 1.7$ & 454.063 & $9.5 \pm 1.7$ & 454.563 & $11.1 \pm 2.1$ \\
\hline 455.063 & $9.3 \pm 1.7$ & 455.563 & $9.7 \pm 1.8$ & 456.063 & $9.6 \pm 1.8$ & 456.563 & $9.4 \pm 1.7$ \\
\hline 457.063 & $9.0 \pm 1.7$ & 457.563 & $8.9 \pm 1.6$ & 458.063 & $8.9 \pm 1.6$ & 458.563 & $9.3 \pm 1.7$ \\
\hline 459.063 & $8.9 \pm 1.6$ & 459.563 & $9.1 \pm 1.7$ & 460.063 & $9.3 \pm 1.7$ & 460.563 & $9.4 \pm 1.7$ \\
\hline 461.063 & $9.4 \pm 1.7$ & 461.563 & $9.3 \pm 1.7$ & 462.063 & $9.3 \pm 1.7$ & 462.563 & $9.3 \pm 1.7$ \\
\hline 463.063 & $9.4 \pm 1.7$ & 463.563 & $9.1 \pm 1.7$ & 464.063 & $9.2 \pm 1.7$ & 464.563 & $9.7 \pm 1.8$ \\
\hline 465.063 & $9.9 \pm 1.8$ & 465.563 & $10.3 \pm 1.9$ & 466.063 & $9.9 \pm 1.8$ & 466.563 & $9.8 \pm 1.8$ \\
\hline 467.063 & $10.2 \pm 1.9$ & 467.563 & $10.1 \pm 1.9$ & 468.063 & $9.8 \pm 1.8$ & 468.563 & $9.6 \pm 1.8$ \\
\hline 469.063 & $9.4 \pm 1.7$ & 469.563 & $9.7 \pm 1.8$ & 470.063 & $9.8 \pm 1.8$ & 470.563 & $9.8 \pm 1.8$ \\
\hline 471.063 & $10.3 \pm 1.9$ & 471.563 & $10.7 \pm 2.0$ & 472.063 & $10.2 \pm 1.9$ & 472.563 & $9.9 \pm 1.8$ \\
\hline 473.063 & $10.2 \pm 1.9$ & 473.563 & $9.9 \pm 1.8$ & 474.063 & $9.7 \pm 1.8$ & 474.563 & $10.0 \pm 1.8$ \\
\hline 475.063 & $9.7 \pm 1.8$ & 475.563 & $10.4 \pm 1.9$ & 476.063 & $10.0 \pm 1.8$ & 476.563 & $9.8 \pm 1.8$ \\
\hline 477.063 & $10.0 \pm 1.8$ & 477.563 & $9.8 \pm 1.8$ & 478.063 & $9.5 \pm 1.7$ & 478.563 & $9.5 \pm 1.7$ \\
\hline 480.563 & $10.3 \pm 1.9$ & 481.063 & $9.7 \pm 1.8$ & 481.563 & $9.8 \pm 1.8$ & 482.063 & $9.7 \pm 1.8$ \\
\hline 482.563 & $9.6 \pm 1.8$ & 483.063 & $9.7 \pm 1.8$ & 483.563 & $9.9 \pm 1.8$ & 484.063 & $9.6 \pm 1.8$ \\
\hline 484.563 & $10.1 \pm 1.8$ & 485.063 & $10.2 \pm 1.9$ & 485.563 & $9.7 \pm 1.8$ & 486.063 & $9.5 \pm 1.7$ \\
\hline 486.563 & $9.8 \pm 1.8$ & 487.063 & $9.5 \pm 1.7$ & 487.563 & $9.5 \pm 1.7$ & 488.063 & $9.5 \pm 1.7$ \\
\hline 488.563 & $9.7 \pm 1.8$ & 489.063 & $10.0 \pm 1.8$ & 489.563 & $10.7 \pm 1.9$ & 490.063 & $10.6 \pm 1.9$ \\
\hline 490.563 & $10.0 \pm 1.8$ & 491.063 & $10.3 \pm 1.9$ & 491.563 & $10.1 \pm 1.9$ & 492.063 & $10.5 \pm 1.9$ \\
\hline 492.563 & $10.5 \pm 1.9$ & 493.063 & $10.8 \pm 2.0$ & 493.563 & $11.7 \pm 2.1$ & 494.063 & $12.6 \pm 2.3$ \\
\hline 494.563 & $11.4 \pm 2.1$ & 495.063 & $12.3 \pm 2.2$ & 495.563 & $11.3 \pm 2.1$ & 496.063 & $10.5 \pm 1.9$ \\
\hline 496.563 & $10.4 \pm 1.9$ & 497.063 & $11.0 \pm 2.1$ & 497.563 & $10.5 \pm 1.9$ & 498.063 & $10.0 \pm 1.8$ \\
\hline 498.563 & $9.8 \pm 1.8$ & 499.063 & $9.7 \pm 1.8$ & 499.563 & $9.9 \pm 1.8$ & 500.063 & $10.2 \pm 1.9$ \\
\hline 500.563 & $10.0 \pm 1.8$ & 501.063 & $9.9 \pm 1.8$ & 501.563 & $9.7 \pm 1.8$ & 502.063 & $9.9 \pm 1.8$ \\
\hline 502.563 & $10.1 \pm 1.9$ & 503.063 & $9.7 \pm 1.8$ & 503.563 & $9.7 \pm 1.8$ & 504.063 & $10.0 \pm 1.8$ \\
\hline 504.563 & $10.1 \pm 1.8$ & 505.063 & $10.2 \pm 1.9$ & 505.563 & $10.4 \pm 1.9$ & 506.063 & $10.0 \pm 1.8$ \\
\hline 506.563 & $9.7 \pm 1.8$ & 507.063 & $10.1 \pm 1.9$ & 507.563 & $9.9 \pm 1.8$ & 508.063 & $9.9 \pm 1.8$ \\
\hline
\end{tabular}

\section{Cleaned-out frequency bins}

TABLE III. Instrumental lines identified and "cleaned" before the Einstein@ Home runs. The different columns represent: (I) the source of the line, (II) the central frequency of the instrumental line, (III) the number of harmonics; (IV) low-frequency-side (LFS) of the knockout band, (V) high-frequency-side (HFS) of the knockout band; (VI) the interferometer where the instrumental lines were identified. Note that when there are higher harmonics, the knockout band width remains constant.

\begin{tabular}{lccccc}
\hline \hline Cause & $f_{\mathrm{L}}(\mathrm{Hz})$ & Harmonics & LFS $(\mathrm{Hz})$ & HFS $(\mathrm{Hz})$ & IFO \\
\hline Mains & 60 & 8 & 1 & 1 & $\mathrm{~L}, \mathrm{H}$ \\
Wire & 345 & 1 & 5 & 5 & $\mathrm{~L}$ \\
Wire & 346 & 1 & 4 & 4 & $\mathrm{H}$ \\
Electronic & 85.8 & 1 & 0.01 & 0.01 & $\mathrm{H}$ \\
Electronic & 89.9 & 1 & 0.06 & 0.06 & $\mathrm{H}$ \\
Electronic & 93.29 & 1 & 0.015 & 0.01 & $\mathrm{~L}$ \\
Electronic & 93.05 & 1 & 0.01 & 0.01 & $\mathrm{H}$ \\
Electronic & 93.25 & 1 & 0.01 & 0.015 & $\mathrm{H}$ \\
Electronic & 96.71 & 1 & 0.015 & 0.02 & $\mathrm{~L}$ \\
Electronic & 139.94 & 1 & 0.02 & 0.01 & $\mathrm{H}$ \\
Electronic & 139.95 & 0.01 & & & \\
\hline
\end{tabular}


TABLE III. (Continued)

\begin{tabular}{|c|c|c|c|c|c|}
\hline Cause & $f_{\mathrm{L}}(\mathrm{Hz})$ & Harmonics & LFS $(\mathrm{Hz})$ & $\mathrm{HFS}(\mathrm{Hz})$ & IFO \\
\hline Electronic & 145.06 & 1 & 0.02 & 0.02 & $\mathrm{~L}$ \\
\hline Electronic & 164.52 & 1 & 0.01 & 0.01 & $\mathrm{H}$ \\
\hline Electronic & 186.59 & 1 & 0.025 & 0.025 & $\mathrm{~L}$ \\
\hline Electronic & 193.42 & 1 & 0.025 & 0.025 & $\mathrm{~L}$ \\
\hline Electronic & 233.23 & 1 & 0.05 & 0.05 & $\mathrm{~L}$ \\
\hline Electronic & 241.78 & 1 & 0.07 & 0.07 & $\mathrm{~L}$ \\
\hline Electronic & 329.58 & 1 & 0.02 & 0.01 & $\mathrm{H}$ \\
\hline Electronic & 329.86 & 1 & 0.01 & 0.02 & $\mathrm{H}$ \\
\hline Violin mode & 329.32 & 1 & 0.11 & 0.11 & $\mathrm{~L}$ \\
\hline Violin mode & 329.70 & 1 & 0.3 & 0.3 & $\mathrm{H}$ \\
\hline Violin mode & 335.53 & 1 & 0.28 & 0.28 & $\mathrm{~L}$ \\
\hline CPU line & 54.496 & 1 & 0.0006 & 0.0006 & $\mathrm{~L}, \mathrm{H}$ \\
\hline CPU line & 108.992 & 1 & 0.0006 & 0.0006 & $\mathrm{~L}, \mathrm{H}$ \\
\hline Sideband comb & 140.4100 & 1 & 0.0006 & 0.0006 & $\mathrm{H}$ \\
\hline Sideband comb & 166.1205 & 1 & 0.0006 & 0.0006 & $\mathrm{H}$ \\
\hline Sideband comb & 191.8322 & 1 & 0.0006 & 0.0006 & $\mathrm{H}$ \\
\hline Sideband comb & 217.5428 & 1 & 0.0006 & 0.0006 & $\mathrm{H}$ \\
\hline Sideband comb & 243.2539 & 1 & 0.0006 & 0.0006 & $\mathrm{H}$ \\
\hline Sideband comb & 268.9650 & 1 & 0.0006 & 0.0006 & $\mathrm{H}$ \\
\hline Sideband comb & 294.6756 & 1 & 0.0006 & 0.0006 & $\mathrm{H}$ \\
\hline Sideband comb & 320.3867 & 1 & 0.0006 & 0.0006 & $\mathrm{H}$ \\
\hline Sideband comb & 346.0972 & 1 & 0.0006 & 0.0006 & $\mathrm{H}$ \\
\hline Sideband comb & 371.8077 & 1 & 0.0006 & 0.0006 & $\mathrm{H}$ \\
\hline Sideband comb & 383.6639 & 1 & 0.0006 & 0.0006 & $\mathrm{H}$ \\
\hline Sideband comb & 397.5194 & 1 & 0.0006 & 0.0006 & $\mathrm{H}$ \\
\hline Sideband comb & 409.3705 & 1 & 0.0006 & 0.0006 & $\mathrm{H}$ \\
\hline Sideband comb & 423.2306 & 1 & 0.0006 & 0.0006 & $\mathrm{H}$ \\
\hline Sideband comb & 435.0861 & 1 & 0.0006 & 0.0006 & $\mathrm{H}$ \\
\hline Sideband comb & 460.7967 & 1 & 0.0006 & 0.0006 & $\mathrm{H}$ \\
\hline Sideband comb & 486.5078 & 1 & 0.0006 & 0.0006 & $\mathrm{H}$ \\
\hline $1 \mathrm{~Hz}$ comb & 1 & 2000 & 0.0006 & 0.0006 & $\mathrm{~L}, \mathrm{H}$ \\
\hline $2 \mathrm{~Hz}$ comb & 52 & 1 & 0.0006 & 0.0006 & $\mathrm{~L}, \mathrm{H}$ \\
\hline $2 \mathrm{~Hz}$ comb & 64 & 1 & 0.0006 & 0.0006 & $\mathrm{~L}, \mathrm{H}$ \\
\hline $2 \mathrm{~Hz}$ comb & 68 & 1 & 0.0006 & 0.0006 & $\mathrm{~L}, \mathrm{H}$ \\
\hline $2 \mathrm{~Hz}$ comb & 76 & 1 & 0.0006 & 0.0006 & $\mathrm{~L}, \mathrm{H}$ \\
\hline $2 \mathrm{~Hz}$ comb & 80 & 1 & 0.0006 & 0.0006 & $\mathrm{~L}, \mathrm{H}$ \\
\hline $2 \mathrm{~Hz}$ comb & 82 & 1 & 0.0006 & 0.0006 & $\mathrm{~L}, \mathrm{H}$ \\
\hline $2 \mathrm{~Hz}$ comb & 90 & 1 & 0.0006 & 0.0006 & $\mathrm{~L}, \mathrm{H}$ \\
\hline $2 \mathrm{~Hz}$ comb & 96 & 1 & 0.0006 & 0.0006 & $\mathrm{~L}, \mathrm{H}$ \\
\hline $2 \mathrm{~Hz}$ comb & 98 & 1 & 0.0006 & 0.0006 & $\mathrm{~L}, \mathrm{H}$ \\
\hline $2 \mathrm{~Hz}$ comb & 102 & 1 & 0.0006 & 0.0006 & $\mathrm{~L}, \mathrm{H}$ \\
\hline $2 \mathrm{~Hz}$ comb & 109 & 1 & 0.0006 & 0.0006 & $\mathrm{~L}, \mathrm{H}$ \\
\hline $2 \mathrm{~Hz}$ comb & 110 & 1 & 0.0006 & 0.0006 & $\mathrm{~L}, \mathrm{H}$ \\
\hline $2 \mathrm{~Hz}$ comb & 111 & 1 & 0.0006 & 0.0006 & $\mathrm{~L}, \mathrm{H}$ \\
\hline $2 \mathrm{~Hz}$ comb & 112 & 1 & 0.0006 & 0.0006 & $\mathrm{~L}, \mathrm{H}$ \\
\hline $2 \mathrm{~Hz}$ comb & 116 & 1 & 0.0006 & 0.0006 & $\mathrm{~L}, \mathrm{H}$ \\
\hline $2 \mathrm{~Hz}$ comb & 120 & 1 & 0.0006 & 0.0006 & $\mathrm{~L}, \mathrm{H}$ \\
\hline $2 \mathrm{~Hz}$ comb & 124 & 1 & 0.0006 & 0.0006 & $\mathrm{~L}, \mathrm{H}$ \\
\hline $2 \mathrm{~Hz}$ comb & 128 & 1 & 0.0006 & 0.0006 & $\mathrm{~L}, \mathrm{H}$ \\
\hline $2 \mathrm{~Hz}$ comb & 132 & 1 & 0.0006 & 0.0006 & $\mathrm{~L}, \mathrm{H}$ \\
\hline $2 \mathrm{~Hz}$ comb & 138 & 1 & 0.0006 & 0.0006 & $\mathrm{~L}, \mathrm{H}$ \\
\hline $2 \mathrm{~Hz}$ comb & 140 & 1 & 0.0006 & 0.0006 & $\mathrm{~L}, \mathrm{H}$ \\
\hline $2 \mathrm{~Hz}$ comb & 142 & 1 & 0.0006 & 0.0006 & $\mathrm{~L}, \mathrm{H}$ \\
\hline $2 \mathrm{~Hz}$ comb & 144 & 1 & 0.0006 & 0.0006 & $\mathrm{~L}, \mathrm{H}$ \\
\hline $2 \mathrm{~Hz}$ comb & 150 & 1 & 0.0006 & 0.0006 & $\mathrm{~L}, \mathrm{H}$ \\
\hline
\end{tabular}


TABLE III. (Continued)

\begin{tabular}{|c|c|c|c|c|c|}
\hline Cause & $f_{\mathrm{L}}(\mathrm{Hz})$ & Harmonics & LFS $(\mathrm{Hz})$ & HFS $(\mathrm{Hz})$ & IFO \\
\hline $2 \mathrm{~Hz}$ comb & 158 & 1 & 0.0006 & 0.0006 & $\mathrm{~L}, \mathrm{H}$ \\
\hline $2 \mathrm{~Hz} \mathrm{comb}$ & 154 & 1 & 0.0006 & 0.0006 & $\mathrm{~L}, \mathrm{H}$ \\
\hline $2 \mathrm{~Hz}$ comb & 162 & 1 & 0.0006 & 0.0006 & $\mathrm{~L}, \mathrm{H}$ \\
\hline $2 \mathrm{~Hz}$ comb & 166 & 1 & 0.0006 & 0.0006 & $\mathrm{~L}, \mathrm{H}$ \\
\hline $2 \mathrm{~Hz}$ comb & 168 & 1 & 0.0006 & 0.0006 & $\mathrm{~L}, \mathrm{H}$ \\
\hline $2 \mathrm{~Hz}$ comb & 170 & 1 & 0.0006 & 0.0006 & $\mathrm{~L}, \mathrm{H}$ \\
\hline $2 \mathrm{~Hz}$ comb & 172 & 1 & 0.0006 & 0.0006 & $\mathrm{~L}, \mathrm{H}$ \\
\hline $2 \mathrm{~Hz}$ comb & 174 & 1 & 0.0006 & 0.0006 & $\mathrm{~L}, \mathrm{H}$ \\
\hline $2 \mathrm{~Hz} \mathrm{comb}$ & 176 & 1 & 0.0006 & 0.0006 & $\mathrm{~L}, \mathrm{H}$ \\
\hline $2 \mathrm{~Hz}$ comb & 178 & 1 & 0.0006 & 0.0006 & $\mathrm{~L}, \mathrm{H}$ \\
\hline $2 \mathrm{~Hz}$ comb & 184 & 1 & 0.0006 & 0.0006 & $\mathrm{~L}, \mathrm{H}$ \\
\hline $2 \mathrm{~Hz}$ comb & 188 & 1 & 0.0006 & 0.0006 & $\mathrm{~L}, \mathrm{H}$ \\
\hline $2 \mathrm{~Hz}$ comb & 192 & 1 & 0.0006 & 0.0006 & $\mathrm{~L}, \mathrm{H}$ \\
\hline $2 \mathrm{~Hz}$ comb & 196 & 1 & 0.0006 & 0.0006 & $\mathrm{~L}, \mathrm{H}$ \\
\hline $2 \mathrm{~Hz}$ comb & 204 & 1 & 0.0006 & 0.0006 & $\mathrm{~L}, \mathrm{H}$ \\
\hline $2 \mathrm{~Hz}$ comb & 206 & 1 & 0.0006 & 0.0006 & $\mathrm{~L}, \mathrm{H}$ \\
\hline $2 \mathrm{~Hz} \mathrm{comb}$ & 214 & 1 & 0.0006 & 0.0006 & $\mathrm{~L}, \mathrm{H}$ \\
\hline $2 \mathrm{~Hz} \mathrm{comb}$ & 216 & 1 & 0.0006 & 0.0006 & $\mathrm{~L}, \mathrm{H}$ \\
\hline $2 \mathrm{~Hz}$ comb & 218 & 1 & 0.0006 & 0.0006 & $\mathrm{~L}, \mathrm{H}$ \\
\hline $2 \mathrm{~Hz}$ comb & 221 & 1 & 0.0006 & 0.0006 & $\mathrm{~L}, \mathrm{H}$ \\
\hline $2 \mathrm{~Hz} \mathrm{comb}$ & 222 & 1 & 0.0006 & 0.0006 & $\mathrm{~L}, \mathrm{H}$ \\
\hline $2 \mathrm{~Hz} \mathrm{comb}$ & 226 & 1 & 0.0006 & 0.0006 & $\mathrm{~L}, \mathrm{H}$ \\
\hline $2 \mathrm{~Hz}$ comb & 234 & 1 & 0.0006 & 0.0006 & $\mathrm{~L}, \mathrm{H}$ \\
\hline $2 \mathrm{~Hz} \mathrm{comb}$ & 236 & 1 & 0.0006 & 0.0006 & $\mathrm{~L}, \mathrm{H}$ \\
\hline $2 \mathrm{~Hz}$ comb & 242 & 1 & 0.0006 & 0.0006 & $\mathrm{~L}, \mathrm{H}$ \\
\hline $2 \mathrm{~Hz} \mathrm{comb}$ & 244 & 1 & 0.0006 & 0.0006 & $\mathrm{~L}, \mathrm{H}$ \\
\hline $2 \mathrm{~Hz}$ comb & 248 & 1 & 0.0006 & 0.0006 & $\mathrm{~L}, \mathrm{H}$ \\
\hline $2 \mathrm{~Hz} \mathrm{comb}$ & 252 & 1 & 0.0006 & 0.0006 & $\mathrm{~L}, \mathrm{H}$ \\
\hline $2 \mathrm{~Hz}$ comb & 254 & 1 & 0.0006 & 0.0006 & $\mathrm{~L}, \mathrm{H}$ \\
\hline $2 \mathrm{~Hz} \mathrm{comb}$ & 256 & 1 & 0.0006 & 0.0006 & $\mathrm{~L}, \mathrm{H}$ \\
\hline $2 \mathrm{~Hz}$ comb & 260 & 1 & 0.0006 & 0.0006 & $\mathrm{~L}, \mathrm{H}$ \\
\hline $2 \mathrm{~Hz} \mathrm{comb}$ & 262 & 1 & 0.0006 & 0.0006 & $\mathrm{~L}, \mathrm{H}$ \\
\hline $2 \mathrm{~Hz}$ comb & 264 & 1 & 0.0006 & 0.0006 & $\mathrm{~L}, \mathrm{H}$ \\
\hline $2 \mathrm{~Hz} \mathrm{comb}$ & 266 & 1 & 0.0006 & 0.0006 & $\mathrm{~L}, \mathrm{H}$ \\
\hline $2 \mathrm{~Hz}$ comb & 268 & 1 & 0.0006 & 0.0006 & $\mathrm{~L}, \mathrm{H}$ \\
\hline $2 \mathrm{~Hz}$ comb & 270 & 1 & 0.0006 & 0.0006 & $\mathrm{~L}, \mathrm{H}$ \\
\hline $2 \mathrm{~Hz}$ comb & 274 & 1 & 0.0006 & 0.0006 & $\mathrm{~L}, \mathrm{H}$ \\
\hline $2 \mathrm{~Hz} \mathrm{comb}$ & 278 & 1 & 0.0006 & 0.0006 & $\mathrm{~L}, \mathrm{H}$ \\
\hline $2 \mathrm{~Hz}$ comb & 280 & 1 & 0.0006 & 0.0006 & $\mathrm{~L}, \mathrm{H}$ \\
\hline $2 \mathrm{~Hz} \mathrm{comb}$ & 282 & 1 & 0.0006 & 0.0006 & $\mathrm{~L}, \mathrm{H}$ \\
\hline $2 \mathrm{~Hz}$ comb & 286 & 1 & 0.0006 & 0.0006 & $\mathrm{~L}, \mathrm{H}$ \\
\hline $2 \mathrm{~Hz} \mathrm{comb}$ & 290 & 1 & 0.0006 & 0.0006 & $\mathrm{~L}, \mathrm{H}$ \\
\hline $2 \mathrm{~Hz}$ comb & 298 & 1 & 0.0006 & 0.0006 & $\mathrm{~L}, \mathrm{H}$ \\
\hline $2 \mathrm{~Hz}$ comb & 308 & 1 & 0.0006 & 0.0006 & $\mathrm{~L}, \mathrm{H}$ \\
\hline $2 \mathrm{~Hz}$ comb & 312 & 1 & 0.0006 & 0.0006 & $\mathrm{~L}, \mathrm{H}$ \\
\hline $2 \mathrm{~Hz} \mathrm{comb}$ & 316 & 1 & 0.0006 & 0.0006 & $\mathrm{~L}, \mathrm{H}$ \\
\hline $2 \mathrm{~Hz}$ comb & 320 & 1 & 0.0006 & 0.0006 & $\mathrm{~L}, \mathrm{H}$ \\
\hline $2 \mathrm{~Hz}$ comb & 334 & 1 & 0.0006 & 0.0006 & $\mathrm{~L}, \mathrm{H}$ \\
\hline $2 \mathrm{~Hz}$ comb & 372 & 1 & 0.0006 & 0.0006 & $\mathrm{~L}, \mathrm{H}$ \\
\hline $2 \mathrm{~Hz} \mathrm{comb}$ & 376 & 1 & 0.0006 & 0.0006 & $\mathrm{~L}, \mathrm{H}$ \\
\hline $2 \mathrm{~Hz}$ comb & 380 & 1 & 0.0006 & 0.0006 & $\mathrm{~L}, \mathrm{H}$ \\
\hline $2 \mathrm{~Hz}$ comb & 384 & 1 & 0.0006 & 0.0006 & $\mathrm{~L}, \mathrm{H}$ \\
\hline $2 \mathrm{~Hz}$ comb & 394 & 1 & 0.0006 & 0.0006 & $\mathrm{~L}, \mathrm{H}$ \\
\hline $2 \mathrm{~Hz}$ comb & 402 & 1 & 0.0006 & 0.0006 & $\mathrm{~L}, \mathrm{H}$ \\
\hline $2 \mathrm{~Hz}$ comb & 410 & 1 & 0.0006 & 0.0006 & $\mathrm{~L}, \mathrm{H}$ \\
\hline
\end{tabular}


TABLE III. (Continued)

\begin{tabular}{|c|c|c|c|c|c|}
\hline Cause & $f_{\mathrm{L}}(\mathrm{Hz})$ & Harmonics & LFS $(\mathrm{Hz})$ & $\mathrm{HFS}(\mathrm{Hz})$ & IFO \\
\hline $2 \mathrm{~Hz}$ comb & 414 & 1 & 0.0006 & 0.0006 & $\mathrm{~L}, \mathrm{H}$ \\
\hline $2 \mathrm{~Hz}$ comb & 418 & 1 & 0.0006 & 0.0006 & $\mathrm{~L}, \mathrm{H}$ \\
\hline $2 \mathrm{~Hz}$ comb & 422 & 1 & 0.0006 & 0.0006 & $\mathrm{~L}, \mathrm{H}$ \\
\hline $2 \mathrm{~Hz}$ comb & 430 & 1 & 0.0006 & 0.0006 & $\mathrm{~L}, \mathrm{H}$ \\
\hline $2 \mathrm{~Hz}$ comb & 432 & 1 & 0.0006 & 0.0006 & $\mathrm{~L}, \mathrm{H}$ \\
\hline $2 \mathrm{~Hz}$ comb & 435 & 1 & 0.0006 & 0.0006 & $\mathrm{~L}, \mathrm{H}$ \\
\hline $2 \mathrm{~Hz}$ comb & 440 & 1 & 0.0006 & 0.0006 & $\mathrm{~L}, \mathrm{H}$ \\
\hline $2 \mathrm{~Hz}$ comb & 448 & 1 & 0.0006 & 0.0006 & $\mathrm{~L}, \mathrm{H}$ \\
\hline $2 \mathrm{~Hz}$ comb & 462 & 1 & 0.0006 & 0.0006 & $\mathrm{~L}, \mathrm{H}$ \\
\hline $2 \mathrm{~Hz}$ comb & 466 & 1 & 0.0006 & 0.0006 & $\mathrm{~L}, \mathrm{H}$ \\
\hline $2 \mathrm{~Hz}$ comb & 468 & 1 & 0.0006 & 0.0006 & $\mathrm{~L}, \mathrm{H}$ \\
\hline $2 \mathrm{~Hz}$ comb & 470 & 1 & 0.0006 & 0.0006 & $\mathrm{~L}, \mathrm{H}$ \\
\hline $2 \mathrm{~Hz}$ comb & 474 & 1 & 0.0006 & 0.0006 & $\mathrm{~L}, \mathrm{H}$ \\
\hline $2 \mathrm{~Hz}$ comb & 482 & 1 & 0.0006 & 0.0006 & $\mathrm{~L}, \mathrm{H}$ \\
\hline $2 \mathrm{~Hz}$ comb & 488 & 1 & 0.0006 & 0.0006 & $\mathrm{~L}, \mathrm{H}$ \\
\hline $2 \mathrm{~Hz}$ comb & 496 & 1 & 0.0006 & 0.0006 & $\mathrm{~L}, \mathrm{H}$ \\
\hline $2 \mathrm{~Hz}$ comb & 500 & 1 & 0.0006 & 0.0006 & $\mathrm{~L}, \mathrm{H}$ \\
\hline $2 \mathrm{~Hz}$ comb & 504 & 1 & 0.0006 & 0.0006 & $\mathrm{~L}, \mathrm{H}$ \\
\hline $2 \mathrm{~Hz}$ comb & 508 & 1 & 0.0006 & 0.0006 & $\mathrm{~L}, \mathrm{H}$ \\
\hline Digital & 55.8 & 1 & 0.05 & 0.05 & $\mathrm{H}$ \\
\hline Digital & 56.875 & 1 & 0.005 & 0.005 & $\mathrm{H}$ \\
\hline Digital & 58.625 & 1 & 0.005 & 0.005 & $\mathrm{H}$ \\
\hline Digital & 69. & 1 & 0.05 & 0.05 & $\mathrm{H}$ \\
\hline Digital & 85.375 & 1 & 0.005 & 0.005 & $\mathrm{H}$ \\
\hline Digital & 113.75 & 1 & 0.01 & 0.01 & $\mathrm{H}$ \\
\hline Digital & 140.24 & 1 & 0.01 & 0.01 & $\mathrm{H}$ \\
\hline Digital & 153.75 & 1 & 0.05 & 0.05 & $\mathrm{H}$ \\
\hline Digital & 158.0 & 1 & 0.05 & 0.05 & $\mathrm{H}$ \\
\hline Digital & 199.57 & 1 & 0.01 & 0.01 & $\mathrm{H}$ \\
\hline Digital & 210.36 & 1 & 0.01 & 0.01 & $\mathrm{H}$ \\
\hline Digital & 373.5 & 1 & 0.05 & 0.05 & $\mathrm{H}$ \\
\hline Digital & 392.2 & 1 & 0.0006 & 0.0006 & $\mathrm{~L}, \mathrm{H}$ \\
\hline Digital & 399.3 & 1 & 0.0006 & 0.0006 & $\mathrm{~L}$ \\
\hline Digital & 401.5 & 1 & 0.05 & 0.05 & $\mathrm{H}$ \\
\hline
\end{tabular}

\section{3. $50 \mathrm{mHz}$ signal-frequency bands that did not contribute to results}

TABLE IV. Signal frequency ranges where the results might have contributions from fake data. When the results are entirely due to artificial data, the band is listed in the "all fake data" column; bands where the results comprise contributions from both fake and real data are listed in the other three columns. The "mixed, left" and "mixed, right" columns are populated only when there is a matching "all fake data" entry, which highlights the same physical cause for the fake data, i.e., the cleaning. The "mixed, isolated" column lists isolated ranges of mixed data. The list of input data frequencies where the data was substituted with artificial noise are given in Table I.

\begin{tabular}{|c|c|c|c|c|c|c|c|c|c|}
\hline \multirow{2}{*}{$\frac{\text { Line type }}{1 \mathrm{~Hz}}$} & \multicolumn{2}{|c|}{ Mixed, isolated } & \multicolumn{2}{|c|}{ Mixed, left } & \multicolumn{2}{|c|}{ All fake data } & \multicolumn{2}{|c|}{ Mixed,right } & \multirow{2}{*}{$\frac{\text { Detector }}{\mathrm{L}, \mathrm{H}}$} \\
\hline & 50.9648 & 51.0352 & & & & & & & \\
\hline $1 \mathrm{~Hz}, 2 \mathrm{~Hz}$ & 51.9647 & 52.0353 & & & & & & & L,H \\
\hline $1 \mathrm{~Hz}$ & 52.9646 & 53.0354 & & & & & & & $\mathrm{~L}, \mathrm{H}$ \\
\hline $1 \mathrm{~Hz}$ & 53.9645 & 54.0355 & & & & & & & $\mathrm{~L}, \mathrm{H}$ \\
\hline CPU & 54.4605 & 54.5315 & & & & & & & $\mathrm{~L}, \mathrm{H}$ \\
\hline $1 \mathrm{~Hz}$ & 54.9644 & 55.0356 & & & & & & & $\mathrm{~L}, \mathrm{H}$ \\
\hline $\mathrm{D}$ & & & 55.715 & 55.785 & 55.785 & 55.815 & 55.815 & 55.885 & $\mathrm{H}$ \\
\hline $1 \mathrm{~Hz}$ & 55.9643 & 56.0357 & & & & & & & $\mathrm{~L}, \mathrm{H}$ \\
\hline
\end{tabular}


TABLE IV. (Continued)

\begin{tabular}{|c|c|c|c|c|c|c|c|c|c|}
\hline \multirow{2}{*}{$\frac{\text { Line type }}{\mathrm{D}}$} & \multicolumn{2}{|c|}{ Mixed, isolated } & \multicolumn{2}{|c|}{ Mixed, left } & \multicolumn{2}{|c|}{ All fake data } & \multicolumn{2}{|c|}{ Mixed,right } & \multirow{2}{*}{$\frac{\text { Detector }}{\mathrm{H}}$} \\
\hline & 56.8348 & 56.9152 & & & & & & & \\
\hline $1 \mathrm{~Hz}$ & 56.9642 & 57.0358 & & & & & & & L,H \\
\hline $1 \mathrm{~Hz}$ & 57.9641 & 58.0359 & & & & & & & $\mathrm{~L}, \mathrm{H}$ \\
\hline $\mathrm{D}$ & 58.5847 & 58.6653 & & & & & & & $\mathrm{H}$ \\
\hline $1 \mathrm{~Hz}, \mathrm{M}$ & & & 58.964 & 59.0348 & 59.0348 & 60.965 & 60.965 & 61.0362 & $\mathrm{~L}, \mathrm{H}$ \\
\hline $1 \mathrm{~Hz}$ & 61.9637 & 62.0363 & & & & & & & $\mathrm{~L}, \mathrm{H}$ \\
\hline $1 \mathrm{~Hz}$ & 62.9636 & 63.0364 & & & & & & & $\mathrm{~L}, \mathrm{H}$ \\
\hline $1 \mathrm{~Hz}, 2 \mathrm{~Hz}$ & 63.9635 & 64.0365 & & & & & & & $\mathrm{~L}, \mathrm{H}$ \\
\hline $1 \mathrm{~Hz}$ & 64.9634 & 65.0366 & & & & & & & $\mathrm{~L}, \mathrm{H}$ \\
\hline $1 \mathrm{~Hz}$ & 65.9633 & 66.0367 & & & & & & & $\mathrm{~L}, \mathrm{H}$ \\
\hline $1 \mathrm{~Hz}$ & 66.9632 & 67.0368 & & & & & & & $\mathrm{~L}, \mathrm{H}$ \\
\hline $1 \mathrm{~Hz}, 2 \mathrm{~Hz}$ & 67.9631 & 68.0369 & & & & & & & $\mathrm{~L}, \mathrm{H}$ \\
\hline $1 \mathrm{~Hz}, \mathrm{D}$ & & & 68.9136 & 68.9864 & 68.9864 & 69.0136 & 69.0136 & 69.0864 & $\mathrm{H}$ \\
\hline $1 \mathrm{~Hz}$ & 68.963 & 69.037 & & & & & & & $\mathrm{~L}$ \\
\hline $1 \mathrm{~Hz}$ & 69.9629 & 70.0371 & & & & & & & $\mathrm{~L}, \mathrm{H}$ \\
\hline $1 \mathrm{~Hz}$ & 70.9628 & 71.0372 & & & & & & & $\mathrm{~L}, \mathrm{H}$ \\
\hline $1 \mathrm{~Hz}$ & 71.9627 & 72.0373 & & & & & & & $\mathrm{~L}, \mathrm{H}$ \\
\hline $1 \mathrm{~Hz}$ & 72.9626 & 73.0374 & & & & & & & $\mathrm{~L}, \mathrm{H}$ \\
\hline $1 \mathrm{~Hz}$ & 73.9625 & 74.0375 & & & & & & & $\mathrm{~L}, \mathrm{H}$ \\
\hline $1 \mathrm{~Hz}$ & 74.9624 & 75.0376 & & & & & & & $\mathrm{~L}, \mathrm{H}$ \\
\hline $1 \mathrm{~Hz}, 2 \mathrm{~Hz}$ & 75.9623 & 76.0377 & & & & & & & $\mathrm{~L}, \mathrm{H}$ \\
\hline $1 \mathrm{~Hz}$ & 76.9622 & 77.0378 & & & & & & & $\mathrm{~L}, \mathrm{H}$ \\
\hline $1 \mathrm{~Hz}$ & 77.9621 & 78.0379 & & & & & & & $\mathrm{~L}, \mathrm{H}$ \\
\hline $1 \mathrm{~Hz}$ & 78.962 & 79.038 & & & & & & & $\mathrm{~L}, \mathrm{H}$ \\
\hline $1 \mathrm{~Hz}, 2 \mathrm{~Hz}$ & 79.9619 & 80.0381 & & & & & & & $\mathrm{~L}, \mathrm{H}$ \\
\hline $1 \mathrm{~Hz}$ & 80.9618 & 81.0382 & & & & & & & $\mathrm{~L}, \mathrm{H}$ \\
\hline $1 \mathrm{~Hz}, 2 \mathrm{~Hz}$ & 81.9617 & 82.0383 & & & & & & & $\mathrm{~L}, \mathrm{H}$ \\
\hline $1 \mathrm{~Hz}$ & 82.9616 & 83.0384 & & & & & & & $\mathrm{~L}, \mathrm{H}$ \\
\hline $1 \mathrm{~Hz}$ & 83.9615 & 84.0385 & & & & & & & $\mathrm{~L}, \mathrm{H}$ \\
\hline $1 \mathrm{~Hz}$ & 84.9614 & 85.0386 & & & & & & & $\mathrm{~L}, \mathrm{H}$ \\
\hline $\mathrm{D}$ & 85.332 & 85.418 & & & & & & & $\mathrm{H}$ \\
\hline $\mathrm{E}$ & 85.752 & 85.848 & & & & & & & $\mathrm{H}$ \\
\hline $1 \mathrm{~Hz}$ & 85.9613 & 86.0387 & & & & & & & $\mathrm{~L}, \mathrm{H}$ \\
\hline $1 \mathrm{~Hz}$ & 86.9612 & 87.0388 & & & & & & & $\mathrm{~L}, \mathrm{H}$ \\
\hline $1 \mathrm{~Hz}$ & 87.9611 & 88.0389 & & & & & & & $\mathrm{~L}, \mathrm{H}$ \\
\hline $1 \mathrm{~Hz}$ & 88.961 & 89.039 & & & & & & & $\mathrm{~L}, \mathrm{H}$ \\
\hline $\mathrm{E}$ & & & 89.8016 & 89.8784 & 89.8784 & 89.9215 & 89.9215 & 89.9985 & $\mathrm{H}$ \\
\hline $1 \mathrm{~Hz}, 2 \mathrm{~Hz}$ & 89.9609 & 90.0391 & & & & & & & $\mathrm{~L}, \mathrm{H}$ \\
\hline $1 \mathrm{~Hz}$ & 90.9608 & 91.0392 & & & & & & & $\mathrm{~L}, \mathrm{H}$ \\
\hline $1 \mathrm{~Hz}$ & 91.9607 & 92.0393 & & & & & & & $\mathrm{~L}, \mathrm{H}$ \\
\hline $1 \mathrm{~Hz}$ & 92.9606 & 93.0394 & & & & & & & $\mathrm{~L}, \mathrm{H}$ \\
\hline E & 93.0012 & 93.0988 & & & & & & & $\mathrm{H}$ \\
\hline $\mathrm{E}$ & 93.2012 & 93.2988 & & & & & & & $\mathrm{H}$ \\
\hline E & 93.2362 & 93.3438 & & & & & & & $\mathrm{~L}$ \\
\hline $1 \mathrm{~Hz}$ & 93.9605 & 94.0395 & & & & & & & $\mathrm{~L}, \mathrm{H}$ \\
\hline $1 \mathrm{~Hz}$ & 94.9604 & 95.0396 & & & & & & & $\mathrm{~L}, \mathrm{H}$ \\
\hline $1 \mathrm{~Hz}, 2 \mathrm{~Hz}$ & 95.9603 & 96.0397 & & & & & & & $\mathrm{~L}, \mathrm{H}$ \\
\hline $\mathrm{E}$ & 96.6559 & 96.7641 & & & & & & & $\mathrm{~L}$ \\
\hline $1 \mathrm{~Hz}$ & 96.9602 & 97.0398 & & & & & & & $\mathrm{~L}, \mathrm{H}$ \\
\hline $1 \mathrm{~Hz}, 2 \mathrm{~Hz}$ & 97.9601 & 98.0399 & & & & & & & $\mathrm{~L}, \mathrm{H}$ \\
\hline $1 \mathrm{~Hz}$ & 98.96 & 99.04 & & & & & & & $\mathrm{~L}, \mathrm{H}$ \\
\hline $1 \mathrm{~Hz}$ & 99.9599 & 100.0401 & & & & & & & $\mathrm{~L}, \mathrm{H}$ \\
\hline $1 \mathrm{~Hz}$ & 100.9598 & 101.0402 & & & & & & & $\mathrm{~L}, \mathrm{H}$ \\
\hline $1 \mathrm{~Hz}, 2 \mathrm{~Hz}$ & 101.9597 & 102.0403 & & & & & & & $\mathrm{~L}, \mathrm{H}$ \\
\hline $1 \mathrm{~Hz}$ & 102.9596 & 103.0404 & & & & & & & $\mathrm{~L}, \mathrm{H}$ \\
\hline
\end{tabular}


TABLE IV. (Continued)

\begin{tabular}{|c|c|c|c|c|c|c|c|c|c|}
\hline \multirow{2}{*}{$\frac{\text { Line type }}{1 \mathrm{~Hz}}$} & \multicolumn{2}{|c|}{ Mixed, isolated } & \multicolumn{2}{|c|}{ Mixed, left } & \multicolumn{2}{|c|}{ All fake data } & \multicolumn{2}{|c|}{ Mixed,right } & \multirow{2}{*}{$\frac{\text { Detector }}{\mathrm{L}, \mathrm{H}}$} \\
\hline & 103.9595 & 104.0405 & & & & & & & \\
\hline $1 \mathrm{~Hz}$ & 104.9594 & 105.0406 & & & & & & & L,H \\
\hline $1 \mathrm{~Hz}$ & 105.9593 & 106.0407 & & & & & & & $\mathrm{~L}, \mathrm{H}$ \\
\hline $1 \mathrm{~Hz}$ & 106.9592 & 107.0408 & & & & & & & $\mathrm{~L}, \mathrm{H}$ \\
\hline $1 \mathrm{~Hz}$ & 107.9591 & 108.0409 & & & & & & & $\mathrm{~L}, \mathrm{H}$ \\
\hline CPU & 108.951 & 109.033 & & & & & & & $\mathrm{~L}, \mathrm{H}$ \\
\hline $1 \mathrm{~Hz}, 2 \mathrm{~Hz}$ & 108.959 & 109.041 & & & & & & & $\mathrm{~L}, \mathrm{H}$ \\
\hline $1 \mathrm{~Hz}, 2 \mathrm{~Hz}$ & 109.9589 & 110.0411 & & & & & & & $\mathrm{~L}, \mathrm{H}$ \\
\hline $1 \mathrm{~Hz}, 2 \mathrm{~Hz}$ & 110.9588 & 111.0412 & & & & & & & $\mathrm{~L}, \mathrm{H}$ \\
\hline $1 \mathrm{~Hz}, 2 \mathrm{~Hz}$ & 111.9587 & 112.0413 & & & & & & & $\mathrm{~L}, \mathrm{H}$ \\
\hline $1 \mathrm{~Hz}$ & 112.9586 & 113.0414 & & & & & & & $\mathrm{~L}, \mathrm{H}$ \\
\hline $\mathrm{D}$ & 113.6992 & 113.8008 & & & & & & & $\mathrm{H}$ \\
\hline $1 \mathrm{~Hz}$ & 113.9585 & 114.0415 & & & & & & & $\mathrm{~L}, \mathrm{H}$ \\
\hline $1 \mathrm{~Hz}$ & 114.9584 & 115.0416 & & & & & & & $\mathrm{~L}, \mathrm{H}$ \\
\hline $1 \mathrm{~Hz}, 2 \mathrm{~Hz}$ & 115.9583 & 116.0417 & & & & & & & $\mathrm{~L}, \mathrm{H}$ \\
\hline $1 \mathrm{~Hz}$ & 116.9582 & 117.0418 & & & & & & & $\mathrm{~L}, \mathrm{H}$ \\
\hline $1 \mathrm{~Hz}$ & 117.9581 & 118.0419 & & & & & & & $\mathrm{~L}, \mathrm{H}$ \\
\hline $1 \mathrm{~Hz}, 2 \mathrm{~Hz}, \mathrm{M}$ & & & 118.958 & 119.0408 & 119.0408 & 120.959 & 120.959 & 121.0422 & $\mathrm{~L}, \mathrm{H}$ \\
\hline $1 \mathrm{~Hz}$ & 121.9577 & 122.0423 & & & & & & & $\mathrm{~L}, \mathrm{H}$ \\
\hline $1 \mathrm{~Hz}$ & 122.9576 & 123.0424 & & & & & & & $\mathrm{~L}, \mathrm{H}$ \\
\hline $1 \mathrm{~Hz}, 2 \mathrm{~Hz}$ & 123.9575 & 124.0425 & & & & & & & $\mathrm{~L}, \mathrm{H}$ \\
\hline $1 \mathrm{~Hz}$ & 124.9574 & 125.0426 & & & & & & & $\mathrm{~L}, \mathrm{H}$ \\
\hline $1 \mathrm{~Hz}$ & 125.9573 & 126.0427 & & & & & & & $\mathrm{~L}, \mathrm{H}$ \\
\hline $1 \mathrm{~Hz}$ & 126.9572 & 127.0428 & & & & & & & $\mathrm{~L}, \mathrm{H}$ \\
\hline $1 \mathrm{~Hz}, 2 \mathrm{~Hz}$ & 127.9571 & 128.0429 & & & & & & & $\mathrm{~L}, \mathrm{H}$ \\
\hline $1 \mathrm{~Hz}$ & 128.957 & 129.043 & & & & & & & $\mathrm{~L}, \mathrm{H}$ \\
\hline $1 \mathrm{~Hz}$ & 129.9569 & 130.0431 & & & & & & & $\mathrm{~L}, \mathrm{H}$ \\
\hline $1 \mathrm{~Hz}$ & 130.9568 & 131.0432 & & & & & & & $\mathrm{~L}, \mathrm{H}$ \\
\hline $1 \mathrm{~Hz}, 2 \mathrm{~Hz}$ & 131.9567 & 132.0433 & & & & & & & $\mathrm{~L}, \mathrm{H}$ \\
\hline $1 \mathrm{~Hz}$ & 132.9566 & 133.0434 & & & & & & & $\mathrm{~L}, \mathrm{H}$ \\
\hline $1 \mathrm{~Hz}$ & 133.9565 & 134.0435 & & & & & & & $\mathrm{~L}, \mathrm{H}$ \\
\hline $1 \mathrm{~Hz}$ & 134.9564 & 135.0436 & & & & & & & $\mathrm{~L}, \mathrm{H}$ \\
\hline $1 \mathrm{~Hz}$ & 135.9563 & 136.0437 & & & & & & & $\mathrm{~L}, \mathrm{H}$ \\
\hline $1 \mathrm{~Hz}$ & 136.9562 & 137.0438 & & & & & & & $\mathrm{~L}, \mathrm{H}$ \\
\hline $1 \mathrm{~Hz}, 2 \mathrm{~Hz}$ & 137.9561 & 138.0439 & & & & & & & $\mathrm{~L}, \mathrm{H}$ \\
\hline $1 \mathrm{~Hz}$ & 138.956 & 139.044 & & & & & & & $\mathrm{~L}, \mathrm{H}$ \\
\hline E & 139.8765 & 140.0035 & & & & & & & $\mathrm{~L}$ \\
\hline $\mathrm{E}$ & 139.8965 & 140.0035 & & & & & & & $\mathrm{H}$ \\
\hline $1 \mathrm{~Hz}, 2 \mathrm{~Hz}$ & 139.9559 & 140.0441 & & & & & & & $\mathrm{~L}, \mathrm{H}$ \\
\hline $\mathrm{D}$ & 140.1865 & 140.2935 & & & & & & & $\mathrm{H}$ \\
\hline SB & 140.3659 & 140.4541 & & & & & & & $\mathrm{H}$ \\
\hline $1 \mathrm{~Hz}$ & 140.9558 & 141.0442 & & & & & & & $\mathrm{~L}, \mathrm{H}$ \\
\hline $1 \mathrm{~Hz}, 2 \mathrm{~Hz}$ & 141.9557 & 142.0443 & & & & & & & $\mathrm{~L}, \mathrm{H}$ \\
\hline $1 \mathrm{~Hz}$ & 142.9556 & 143.0444 & & & & & & & $\mathrm{~L}, \mathrm{H}$ \\
\hline $1 \mathrm{~Hz}, 2 \mathrm{~Hz}$ & 143.9555 & 144.0445 & & & & & & & $\mathrm{~L}, \mathrm{H}$ \\
\hline $1 \mathrm{~Hz}$ & 144.9554 & 145.0446 & & & & & & & $\mathrm{~L}, \mathrm{H}$ \\
\hline E & 144.996 & 145.124 & & & & & & & $\mathrm{~L}$ \\
\hline $1 \mathrm{~Hz}$ & 145.9553 & 146.0447 & & & & & & & $\mathrm{~L}, \mathrm{H}$ \\
\hline $1 \mathrm{~Hz}$ & 146.9552 & 147.0448 & & & & & & & $\mathrm{~L}, \mathrm{H}$ \\
\hline $1 \mathrm{~Hz}$ & 147.9551 & 148.0449 & & & & & & & $\mathrm{~L}, \mathrm{H}$ \\
\hline $1 \mathrm{~Hz}$ & 148.955 & 149.045 & & & & & & & $\mathrm{~L}, \mathrm{H}$ \\
\hline $1 \mathrm{~Hz}, 2 \mathrm{~Hz}$ & 149.9549 & 150.0451 & & & & & & & $\mathrm{~L}, \mathrm{H}$ \\
\hline $1 \mathrm{~Hz}$ & 150.9548 & 151.0452 & & & & & & & $\mathrm{~L}, \mathrm{H}$ \\
\hline $1 \mathrm{~Hz}$ & 151.9547 & 152.0453 & & & & & & & $\mathrm{~L}, \mathrm{H}$ \\
\hline $1 \mathrm{~Hz}$ & 152.9546 & 153.0454 & & & & & & & $\mathrm{~L}, \mathrm{H}$ \\
\hline
\end{tabular}


TABLE IV. (Continued)

\begin{tabular}{|c|c|c|c|c|c|c|c|c|c|}
\hline \multirow{2}{*}{$\frac{\text { Line type }}{\mathrm{D}}$} & \multicolumn{2}{|c|}{ Mixed, isolated } & \multicolumn{2}{|c|}{ Mixed, left } & \multicolumn{2}{|c|}{ All fake data } & \multicolumn{2}{|c|}{ Mixed,right } & \multirow{2}{*}{$\frac{\text { Detecto } 1}{\mathrm{H}}$} \\
\hline & & & 153.6552 & 153.7448 & 153.7448 & 153.7552 & 153.7552 & 153.8448 & \\
\hline $1 \mathrm{~Hz}, 2 \mathrm{~Hz}$ & 153.9545 & 154.0455 & & & & & & & $\mathrm{~L}, \mathrm{H}$ \\
\hline $1 \mathrm{~Hz}$ & 154.9544 & 155.0456 & & & & & & & $\mathrm{~L}, \mathrm{H}$ \\
\hline $1 \mathrm{~Hz}$ & 155.9543 & 156.0457 & & & & & & & $\mathrm{~L}, \mathrm{H}$ \\
\hline $1 \mathrm{~Hz}$ & 156.9542 & 157.0458 & & & & & & & $\mathrm{~L}, \mathrm{H}$ \\
\hline $1 \mathrm{~Hz}, 2 \mathrm{~Hz}, \mathrm{D}$ & & & 157.9047 & 157.9953 & 157.9953 & 158.0047 & 158.0047 & 158.0953 & $\mathrm{H}$ \\
\hline $1 \mathrm{~Hz}, 2 \mathrm{~Hz}$ & 157.9541 & 158.0459 & & & & & & & $\mathrm{~L}$ \\
\hline $1 \mathrm{~Hz}$ & 158.954 & 159.046 & & & & & & & $\mathrm{~L}, \mathrm{H}$ \\
\hline $1 \mathrm{~Hz}$ & 159.9539 & 160.0461 & & & & & & & $\mathrm{~L}, \mathrm{H}$ \\
\hline $1 \mathrm{~Hz}$ & 160.9538 & 161.0462 & & & & & & & $\mathrm{~L}, \mathrm{H}$ \\
\hline $1 \mathrm{~Hz}, 2 \mathrm{~Hz}$ & 161.9537 & 162.0463 & & & & & & & $\mathrm{~L}, \mathrm{H}$ \\
\hline $1 \mathrm{~Hz}$ & 162.9536 & 163.0464 & & & & & & & $\mathrm{~L}, \mathrm{H}$ \\
\hline $1 \mathrm{~Hz}$ & 163.9535 & 164.0465 & & & & & & & $\mathrm{~L}, \mathrm{H}$ \\
\hline $\mathrm{E}$ & 164.4641 & 164.5759 & & & & & & & $\mathrm{H}$ \\
\hline $1 \mathrm{~Hz}$ & 164.9534 & 165.0466 & & & & & & & $\mathrm{~L}, \mathrm{H}$ \\
\hline $1 \mathrm{~Hz}, 2 \mathrm{~Hz}$ & 165.9533 & 166.0467 & & & & & & & $\mathrm{~L}, \mathrm{H}$ \\
\hline SB & 166.0738 & 166.1672 & & & & & & & $\mathrm{H}$ \\
\hline $1 \mathrm{~Hz}$ & 166.9532 & 167.0468 & & & & & & & $\mathrm{~L}, \mathrm{H}$ \\
\hline $1 \mathrm{~Hz}, 2 \mathrm{~Hz}$ & 167.9531 & 168.0469 & & & & & & & $\mathrm{~L}, \mathrm{H}$ \\
\hline $1 \mathrm{~Hz}$ & 168.953 & 169.047 & & & & & & & $\mathrm{~L}, \mathrm{H}$ \\
\hline $1 \mathrm{~Hz}, 2 \mathrm{~Hz}$ & 169.9529 & 170.0471 & & & & & & & $\mathrm{~L}, \mathrm{H}$ \\
\hline $1 \mathrm{~Hz}$ & 170.9528 & 171.0472 & & & & & & & $\mathrm{~L}, \mathrm{H}$ \\
\hline $1 \mathrm{~Hz}, 2 \mathrm{~Hz}$ & 171.9527 & 172.0473 & & & & & & & $\mathrm{~L}, \mathrm{H}$ \\
\hline $1 \mathrm{~Hz}$ & 172.9526 & 173.0474 & & & & & & & $\mathrm{~L}, \mathrm{H}$ \\
\hline $1 \mathrm{~Hz}, 2 \mathrm{~Hz}$ & 173.9525 & 174.0475 & & & & & & & $\mathrm{~L}, \mathrm{H}$ \\
\hline $1 \mathrm{~Hz}$ & 174.9524 & 175.0476 & & & & & & & $\mathrm{~L}, \mathrm{H}$ \\
\hline $1 \mathrm{~Hz}, 2 \mathrm{~Hz}$ & 175.9523 & 176.0477 & & & & & & & $\mathrm{~L}, \mathrm{H}$ \\
\hline $1 \mathrm{~Hz}$ & 176.9522 & 177.0478 & & & & & & & $\mathrm{~L}, \mathrm{H}$ \\
\hline $1 \mathrm{~Hz}, 2 \mathrm{~Hz}$ & 177.9521 & 178.0479 & & & & & & & $\mathrm{~L}, \mathrm{H}$ \\
\hline $1 \mathrm{~Hz}, \mathrm{M}$ & & & 178.952 & 179.0468 & 179.0468 & 180.953 & 180.953 & 181.0482 & $\mathrm{~L}, \mathrm{H}$ \\
\hline $1 \mathrm{~Hz}$ & 181.9517 & 182.0483 & & & & & & & $\mathrm{~L}, \mathrm{H}$ \\
\hline $1 \mathrm{~Hz}$ & 182.9516 & 183.0484 & & & & & & & $\mathrm{~L}, \mathrm{H}$ \\
\hline $1 \mathrm{~Hz}, 2 \mathrm{~Hz}$ & 183.9515 & 184.0485 & & & & & & & $\mathrm{~L}, \mathrm{H}$ \\
\hline $1 \mathrm{~Hz}$ & 184.9514 & 185.0486 & & & & & & & $\mathrm{~L}, \mathrm{H}$ \\
\hline $1 \mathrm{~Hz}$ & 185.9513 & 186.0487 & & & & & & & $\mathrm{~L}, \mathrm{H}$ \\
\hline $\mathrm{E}$ & 186.5169 & 186.6631 & & & & & & & $\mathrm{~L}$ \\
\hline $1 \mathrm{~Hz}$ & 186.9512 & 187.0488 & & & & & & & $\mathrm{~L}, \mathrm{H}$ \\
\hline $1 \mathrm{~Hz}, 2 \mathrm{~Hz}$ & 187.9511 & 188.0489 & & & & & & & $\mathrm{~L}, \mathrm{H}$ \\
\hline $1 \mathrm{~Hz}$ & 188.951 & 189.049 & & & & & & & $\mathrm{~L}, \mathrm{H}$ \\
\hline $1 \mathrm{~Hz}$ & 189.9509 & 190.0491 & & & & & & & $\mathrm{~L}, \mathrm{H}$ \\
\hline $1 \mathrm{~Hz}$ & 190.9508 & 191.0492 & & & & & & & $\mathrm{~L}, \mathrm{H}$ \\
\hline SB & 191.783 & 191.8814 & & & & & & & $\mathrm{H}$ \\
\hline $1 \mathrm{~Hz}, 2 \mathrm{~Hz}$ & 191.9507 & 192.0493 & & & & & & & $\mathrm{~L}, \mathrm{H}$ \\
\hline $1 \mathrm{~Hz}$ & 192.9506 & 193.0494 & & & & & & & $\mathrm{~L}, \mathrm{H}$ \\
\hline $\mathrm{E}$ & 193.3462 & 193.4938 & & & & & & & $\mathrm{~L}$ \\
\hline $1 \mathrm{~Hz}$ & 193.9505 & 194.0495 & & & & & & & $\mathrm{~L}, \mathrm{H}$ \\
\hline $1 \mathrm{~Hz}$ & 194.9504 & 195.0496 & & & & & & & $\mathrm{~L}, \mathrm{H}$ \\
\hline $1 \mathrm{~Hz}, 2 \mathrm{~Hz}$ & 195.9503 & 196.0497 & & & & & & & $\mathrm{~L}, \mathrm{H}$ \\
\hline $1 \mathrm{~Hz}$ & 196.9502 & 197.0498 & & & & & & & $\mathrm{~L}, \mathrm{H}$ \\
\hline $1 \mathrm{~Hz}$ & 197.9501 & 198.0499 & & & & & & & $\mathrm{~L}, \mathrm{H}$ \\
\hline $1 \mathrm{~Hz}$ & 198.95 & 199.05 & & & & & & & $\mathrm{~L}, \mathrm{H}$ \\
\hline $\mathrm{D}$ & 199.5106 & 199.6294 & & & & & & & $\mathrm{H}$ \\
\hline $1 \mathrm{~Hz}$ & 199.9499 & 200.0501 & & & & & & & $\mathrm{~L}, \mathrm{H}$ \\
\hline $1 \mathrm{~Hz}$ & 200.9498 & 201.0502 & & & & & & & $\mathrm{~L}, \mathrm{H}$ \\
\hline $1 \mathrm{~Hz}$ & 201.9497 & 202.0503 & & & & & & & $\mathrm{~L}, \mathrm{H}$ \\
\hline
\end{tabular}


TABLE IV. (Continued)

\begin{tabular}{|c|c|c|c|c|c|c|c|c|c|}
\hline \multirow{2}{*}{$\frac{\text { Line type }}{1 \mathrm{~Hz}}$} & \multicolumn{2}{|c|}{ Mixed, isolated } & \multicolumn{2}{|c|}{ Mixed, left } & \multicolumn{2}{|c|}{ All fake data } & \multicolumn{2}{|c|}{ Mixed,right } & \multirow{2}{*}{$\frac{\text { Detector }}{\mathrm{L}, \mathrm{H}}$} \\
\hline & 202.9496 & 203.0504 & & & & & & & \\
\hline $1 \mathrm{~Hz}, 2 \mathrm{~Hz}$ & 203.9495 & 204.0505 & & & & & & & L,H \\
\hline $1 \mathrm{~Hz}$ & 204.9494 & 205.0506 & & & & & & & $\mathrm{~L}, \mathrm{H}$ \\
\hline $1 \mathrm{~Hz}, 2 \mathrm{~Hz}$ & 205.9493 & 206.0507 & & & & & & & $\mathrm{~L}, \mathrm{H}$ \\
\hline $1 \mathrm{~Hz}$ & 206.9492 & 207.0508 & & & & & & & $\mathrm{~L}, \mathrm{H}$ \\
\hline $1 \mathrm{~Hz}$ & 207.9491 & 208.0509 & & & & & & & $\mathrm{~L}, \mathrm{H}$ \\
\hline $1 \mathrm{~Hz}$ & 208.949 & 209.051 & & & & & & & $\mathrm{~L}, \mathrm{H}$ \\
\hline $1 \mathrm{~Hz}$ & 209.9489 & 210.0511 & & & & & & & $\mathrm{~L}, \mathrm{H}$ \\
\hline $\mathrm{D}$ & 210.2995 & 210.4205 & & & & & & & $\mathrm{H}$ \\
\hline $1 \mathrm{~Hz}$ & 210.9488 & 211.0512 & & & & & & & $\mathrm{~L}, \mathrm{H}$ \\
\hline $1 \mathrm{~Hz}$ & 211.9487 & 212.0513 & & & & & & & $\mathrm{~L}, \mathrm{H}$ \\
\hline $1 \mathrm{~Hz}$ & 212.9486 & 213.0514 & & & & & & & $\mathrm{~L}, \mathrm{H}$ \\
\hline $1 \mathrm{~Hz}, 2 \mathrm{~Hz}$ & 213.9485 & 214.0515 & & & & & & & $\mathrm{~L}, \mathrm{H}$ \\
\hline $1 \mathrm{~Hz}$ & 214.9484 & 215.0516 & & & & & & & $\mathrm{~L}, \mathrm{H}$ \\
\hline $1 \mathrm{~Hz}, 2 \mathrm{~Hz}$ & 215.9483 & 216.0517 & & & & & & & $\mathrm{~L}, \mathrm{H}$ \\
\hline $1 \mathrm{~Hz}$ & 216.9482 & 217.0518 & & & & & & & $\mathrm{~L}, \mathrm{H}$ \\
\hline SB & 217.491 & 217.5946 & & & & & & & $\mathrm{H}$ \\
\hline $1 \mathrm{~Hz}, 2 \mathrm{~Hz}$ & 217.9481 & 218.0519 & & & & & & & $\mathrm{~L}, \mathrm{H}$ \\
\hline $1 \mathrm{~Hz}$ & 218.948 & 219.052 & & & & & & & $\mathrm{~L}, \mathrm{H}$ \\
\hline $1 \mathrm{~Hz}$ & 219.9479 & 220.0521 & & & & & & & $\mathrm{~L}, \mathrm{H}$ \\
\hline $1 \mathrm{~Hz}, 2 \mathrm{~Hz}$ & 220.9478 & 221.0522 & & & & & & & $\mathrm{~L}, \mathrm{H}$ \\
\hline $1 \mathrm{~Hz}, 2 \mathrm{~Hz}$ & 221.9477 & 222.0523 & & & & & & & $\mathrm{~L}, \mathrm{H}$ \\
\hline $1 \mathrm{~Hz}$ & 222.9476 & 223.0524 & & & & & & & $\mathrm{~L}, \mathrm{H}$ \\
\hline $1 \mathrm{~Hz}$ & 223.9475 & 224.0525 & & & & & & & $\mathrm{~L}, \mathrm{H}$ \\
\hline $1 \mathrm{~Hz}$ & 224.9474 & 225.0526 & & & & & & & $\mathrm{~L}, \mathrm{H}$ \\
\hline $1 \mathrm{~Hz}, 2 \mathrm{~Hz}$ & 225.9473 & 226.0527 & & & & & & & $\mathrm{~L}, \mathrm{H}$ \\
\hline $1 \mathrm{~Hz}$ & 226.9472 & 227.0528 & & & & & & & $\mathrm{~L}, \mathrm{H}$ \\
\hline $1 \mathrm{~Hz}$ & 227.9471 & 228.0529 & & & & & & & $\mathrm{~L}, \mathrm{H}$ \\
\hline $1 \mathrm{~Hz}$ & 228.947 & 229.053 & & & & & & & $\mathrm{~L}, \mathrm{H}$ \\
\hline $1 \mathrm{~Hz}$ & 229.9469 & 230.0531 & & & & & & & $\mathrm{~L}, \mathrm{H}$ \\
\hline $1 \mathrm{~Hz}$ & 230.9468 & 231.0532 & & & & & & & $\mathrm{~L}, \mathrm{H}$ \\
\hline $1 \mathrm{~Hz}$ & 231.9467 & 232.0533 & & & & & & & $\mathrm{~L}, \mathrm{H}$ \\
\hline $1 \mathrm{~Hz}$ & 232.9466 & 233.0534 & & & & & & & $\mathrm{~L}, \mathrm{H}$ \\
\hline $\mathrm{E}$ & 233.1272 & 233.3328 & & & & & & & $\mathrm{~L}$ \\
\hline $1 \mathrm{~Hz}, 2 \mathrm{~Hz}$ & 233.9465 & 234.0535 & & & & & & & $\mathrm{~L}, \mathrm{H}$ \\
\hline $1 \mathrm{~Hz}$ & 234.9464 & 235.0536 & & & & & & & $\mathrm{~L}, \mathrm{H}$ \\
\hline $1 \mathrm{~Hz}, 2 \mathrm{~Hz}$ & 235.9463 & 236.0537 & & & & & & & $\mathrm{~L}, \mathrm{H}$ \\
\hline $1 \mathrm{~Hz}$ & 236.9462 & 237.0538 & & & & & & & $\mathrm{~L}, \mathrm{H}$ \\
\hline $1 \mathrm{~Hz}$ & 237.9461 & 238.0539 & & & & & & & $\mathrm{~L}, \mathrm{H}$ \\
\hline $1 \mathrm{~Hz}, \mathrm{M}$ & & & 238.946 & 239.0528 & 239.0528 & 240.947 & 240.947 & 241.0542 & $\mathrm{~L}, \mathrm{H}$ \\
\hline $\mathrm{E}$ & & & 241.6564 & 241.7636 & 241.7636 & 241.7964 & 241.7964 & 241.9036 & $\mathrm{~L}$ \\
\hline $1 \mathrm{~Hz}, 2 \mathrm{~Hz}$ & 241.9457 & 242.0543 & & & & & & & $\mathrm{~L}, \mathrm{H}$ \\
\hline $1 \mathrm{~Hz}$ & 242.9456 & 243.0544 & & & & & & & $\mathrm{~L}, \mathrm{H}$ \\
\hline SB & 243.1995 & 243.3083 & & & & & & & $\mathrm{H}$ \\
\hline $1 \mathrm{~Hz}, 2 \mathrm{~Hz}$ & 243.9455 & 244.0545 & & & & & & & $\mathrm{~L}, \mathrm{H}$ \\
\hline $1 \mathrm{~Hz}$ & 244.9454 & 245.0546 & & & & & & & $\mathrm{~L}, \mathrm{H}$ \\
\hline $1 \mathrm{~Hz}$ & 245.9453 & 246.0547 & & & & & & & $\mathrm{~L}, \mathrm{H}$ \\
\hline $1 \mathrm{~Hz}$ & 246.9452 & 247.0548 & & & & & & & $\mathrm{~L}, \mathrm{H}$ \\
\hline $1 \mathrm{~Hz}, 2 \mathrm{~Hz}$ & 247.9451 & 248.0549 & & & & & & & $\mathrm{~L}, \mathrm{H}$ \\
\hline $1 \mathrm{~Hz}$ & 248.945 & 249.055 & & & & & & & $\mathrm{~L}, \mathrm{H}$ \\
\hline $1 \mathrm{~Hz}$ & 249.9449 & 250.0551 & & & & & & & $\mathrm{~L}, \mathrm{H}$ \\
\hline $1 \mathrm{~Hz}$ & 250.9448 & 251.0552 & & & & & & & $\mathrm{~L}, \mathrm{H}$ \\
\hline $1 \mathrm{~Hz}, 2 \mathrm{~Hz}$ & 251.9447 & 252.0553 & & & & & & & $\mathrm{~L}, \mathrm{H}$ \\
\hline $1 \mathrm{~Hz}$ & 252.9446 & 253.0554 & & & & & & & $\mathrm{~L}, \mathrm{H}$ \\
\hline $1 \mathrm{~Hz}, 2 \mathrm{~Hz}$ & 253.9445 & 254.0555 & & & & & & & $\mathrm{~L}, \mathrm{H}$ \\
\hline
\end{tabular}


TABLE IV. (Continued)

\begin{tabular}{|c|c|c|c|c|c|c|c|c|}
\hline \multirow{2}{*}{$\frac{\text { Line type }}{1 \mathrm{~Hz}}$} & \multicolumn{2}{|c|}{ Mixed, isolated } & Mixed, left & \multicolumn{2}{|c|}{ All fake data } & \multicolumn{2}{|c|}{ Mixed,right } & \multirow{2}{*}{$\frac{\text { Detector }}{\mathrm{L}, \mathrm{H}}$} \\
\hline & 254.9444 & 255.0556 & & & & & & \\
\hline $1 \mathrm{~Hz}, 2 \mathrm{~Hz}$ & 255.9443 & 256.0557 & & & & & & $\mathrm{~L}, \mathrm{H}$ \\
\hline $1 \mathrm{~Hz}$ & 256.9442 & 257.0558 & & & & & & $\mathrm{~L}, \mathrm{H}$ \\
\hline $1 \mathrm{~Hz}$ & 257.9441 & 258.0559 & & & & & & $\mathrm{~L}, \mathrm{H}$ \\
\hline $1 \mathrm{~Hz}$ & 258.944 & 259.056 & & & & & & $\mathrm{~L}, \mathrm{H}$ \\
\hline $1 \mathrm{~Hz}, 2 \mathrm{~Hz}$ & 259.9439 & 260.0561 & & & & & & $\mathrm{~L}, \mathrm{H}$ \\
\hline $1 \mathrm{~Hz}$ & 260.9438 & 261.0562 & & & & & & $\mathrm{~L}, \mathrm{H}$ \\
\hline $1 \mathrm{~Hz}, 2 \mathrm{~Hz}$ & 261.9437 & 262.0563 & & & & & & $\mathrm{~L}, \mathrm{H}$ \\
\hline $1 \mathrm{~Hz}$ & 262.9436 & 263.0564 & & & & & & $\mathrm{~L}, \mathrm{H}$ \\
\hline $1 \mathrm{~Hz}, 2 \mathrm{~Hz}$ & 263.9435 & 264.0565 & & & & & & $\mathrm{~L}, \mathrm{H}$ \\
\hline $1 \mathrm{~Hz}$ & 264.9434 & 265.0566 & & & & & & $\mathrm{~L}, \mathrm{H}$ \\
\hline $1 \mathrm{~Hz}, 2 \mathrm{~Hz}$ & 265.9433 & 266.0567 & & & & & & $\mathrm{~L}, \mathrm{H}$ \\
\hline $1 \mathrm{~Hz}$ & 266.9432 & 267.0568 & & & & & & $\mathrm{~L}, \mathrm{H}$ \\
\hline $1 \mathrm{~Hz}, 2 \mathrm{~Hz}$ & 267.9431 & 268.0569 & & & & & & $\mathrm{~L}, \mathrm{H}$ \\
\hline SB & 268.908 & 269.022 & & & & & & $\mathrm{H}$ \\
\hline $1 \mathrm{~Hz}$ & 268.943 & 269.057 & & & & & & $\mathrm{~L}, \mathrm{H}$ \\
\hline $1 \mathrm{~Hz}, 2 \mathrm{~Hz}$ & 269.9429 & 270.0571 & & & & & & $\mathrm{~L}, \mathrm{H}$ \\
\hline $1 \mathrm{~Hz}$ & 270.9428 & 271.0572 & & & & & & $\mathrm{~L}, \mathrm{H}$ \\
\hline $1 \mathrm{~Hz}$ & 271.9427 & 272.0573 & & & & & & $\mathrm{~L}, \mathrm{H}$ \\
\hline $1 \mathrm{~Hz}$ & 272.9426 & 273.0574 & & & & & & $\mathrm{~L}, \mathrm{H}$ \\
\hline $1 \mathrm{~Hz}, 2 \mathrm{~Hz}$ & 273.9425 & 274.0575 & & & & & & $\mathrm{~L}, \mathrm{H}$ \\
\hline $1 \mathrm{~Hz}$ & 274.9424 & 275.0576 & & & & & & $\mathrm{~L}, \mathrm{H}$ \\
\hline $1 \mathrm{~Hz}$ & 275.9423 & 276.0577 & & & & & & $\mathrm{~L}, \mathrm{H}$ \\
\hline $1 \mathrm{~Hz}$ & 276.9422 & 277.0578 & & & & & & $\mathrm{~L}, \mathrm{H}$ \\
\hline $1 \mathrm{~Hz}, 2 \mathrm{~Hz}$ & 277.9421 & 278.0579 & & & & & & $\mathrm{~L}, \mathrm{H}$ \\
\hline $1 \mathrm{~Hz}$ & 278.942 & 279.058 & & & & & & $\mathrm{~L}, \mathrm{H}$ \\
\hline $1 \mathrm{~Hz}, 2 \mathrm{~Hz}$ & 279.9419 & 280.0581 & & & & & & $\mathrm{~L}, \mathrm{H}$ \\
\hline $1 \mathrm{~Hz}$ & 280.9418 & 281.0582 & & & & & & $\mathrm{~L}, \mathrm{H}$ \\
\hline $1 \mathrm{~Hz}, 2 \mathrm{~Hz}$ & 281.9417 & 282.0583 & & & & & & $\mathrm{~L}, \mathrm{H}$ \\
\hline $1 \mathrm{~Hz}$ & 282.9416 & 283.0584 & & & & & & $\mathrm{~L}, \mathrm{H}$ \\
\hline $1 \mathrm{~Hz}$ & 283.9415 & 284.0585 & & & & & & $\mathrm{~L}, \mathrm{H}$ \\
\hline $1 \mathrm{~Hz}$ & 284.9414 & 285.0586 & & & & & & $\mathrm{~L}, \mathrm{H}$ \\
\hline $1 \mathrm{~Hz}, 2 \mathrm{~Hz}$ & 285.9413 & 286.0587 & & & & & & $\mathrm{~L}, \mathrm{H}$ \\
\hline $1 \mathrm{~Hz}$ & 286.9412 & 287.0588 & & & & & & $\mathrm{~L}, \mathrm{H}$ \\
\hline $1 \mathrm{~Hz}$ & 287.9411 & 288.0589 & & & & & & $\mathrm{~L}, \mathrm{H}$ \\
\hline $1 \mathrm{~Hz}$ & 288.941 & 289.059 & & & & & & $\mathrm{~L}, \mathrm{H}$ \\
\hline $1 \mathrm{~Hz}, 2 \mathrm{~Hz}$ & 289.9409 & 290.0591 & & & & & & $\mathrm{~L}, \mathrm{H}$ \\
\hline $1 \mathrm{~Hz}$ & 290.9408 & 291.0592 & & & & & & $\mathrm{~L}, \mathrm{H}$ \\
\hline $1 \mathrm{~Hz}$ & 291.9407 & 292.0593 & & & & & & $\mathrm{~L}, \mathrm{H}$ \\
\hline $1 \mathrm{~Hz}$ & 292.9406 & 293.0594 & & & & & & $\mathrm{~L}, \mathrm{H}$ \\
\hline $1 \mathrm{~Hz}$ & 293.9405 & 294.0595 & & & & & & $\mathrm{~L}, \mathrm{H}$ \\
\hline SB & 294.6161 & 294.7351 & & & & & & $\mathrm{H}$ \\
\hline $1 \mathrm{~Hz}$ & 294.9404 & 295.0596 & & & & & & $\mathrm{~L}, \mathrm{H}$ \\
\hline $1 \mathrm{~Hz}$ & 295.9403 & 296.0597 & & & & & & $\mathrm{~L}, \mathrm{H}$ \\
\hline $1 \mathrm{~Hz}$ & 296.9402 & 297.0598 & & & & & & $\mathrm{~L}, \mathrm{H}$ \\
\hline $1 \mathrm{~Hz}, 2 \mathrm{~Hz}$ & 297.9401 & 298.0599 & & & & & & $\mathrm{~L}, \mathrm{H}$ \\
\hline $1 \mathrm{~Hz}, \mathrm{M}$ & & & 299.0588 & 299.0588 & 300.941 & 300.941 & 301.0602 & $\mathrm{~L}, \mathrm{H}$ \\
\hline $1 \mathrm{~Hz}$ & 301.9397 & 302.0603 & & & & & & $\mathrm{~L}, \mathrm{H}$ \\
\hline $1 \mathrm{~Hz}$ & 302.9396 & 303.0604 & & & & & & $\mathrm{~L}, \mathrm{H}$ \\
\hline $1 \mathrm{~Hz}$ & 303.9395 & 304.0605 & & & & & & $\mathrm{~L}, \mathrm{H}$ \\
\hline $1 \mathrm{~Hz}$ & 304.9394 & 305.0606 & & & & & & $\mathrm{~L}, \mathrm{H}$ \\
\hline $1 \mathrm{~Hz}$ & 305.9393 & 306.0607 & & & & & & $\mathrm{~L}, \mathrm{H}$ \\
\hline $1 \mathrm{~Hz}$ & 306.9392 & 307.0608 & & & & & & $\mathrm{~L}, \mathrm{H}$ \\
\hline $1 \mathrm{~Hz}, 2 \mathrm{~Hz}$ & 307.9391 & 308.0609 & & & & & & $\mathrm{~L}, \mathrm{H}$ \\
\hline $1 \mathrm{~Hz}$ & 308.939 & 309.061 & & & & & & $\mathrm{~L}, \mathrm{H}$ \\
\hline
\end{tabular}


TABLE IV. (Continued)

\begin{tabular}{|c|c|c|c|c|c|c|c|c|c|}
\hline \multirow{2}{*}{$\frac{\text { Line type }}{1 \mathrm{~Hz}}$} & \multicolumn{2}{|c|}{ Mixed, isolated } & \multicolumn{2}{|c|}{ Mixed, left } & \multicolumn{2}{|c|}{ All fake data } & \multicolumn{2}{|c|}{ Mixed,right } & \multirow{2}{*}{$\frac{\text { Detector }}{\mathrm{L}, \mathrm{H}}$} \\
\hline & 309.9389 & 310.0611 & & & & & & & \\
\hline $1 \mathrm{~Hz}$ & 310.9388 & 311.0612 & & & & & & & $\mathrm{~L}, \mathrm{H}$ \\
\hline $1 \mathrm{~Hz}, 2 \mathrm{~Hz}$ & 311.9387 & 312.0613 & & & & & & & $\mathrm{~L}, \mathrm{H}$ \\
\hline $1 \mathrm{~Hz}$ & 312.9386 & 313.0614 & & & & & & & $\mathrm{~L}, \mathrm{H}$ \\
\hline $1 \mathrm{~Hz}$ & 313.9385 & 314.0615 & & & & & & & $\mathrm{~L}, \mathrm{H}$ \\
\hline $1 \mathrm{~Hz}$ & 314.9384 & 315.0616 & & & & & & & $\mathrm{~L}, \mathrm{H}$ \\
\hline $1 \mathrm{~Hz}, 2 \mathrm{~Hz}$ & 315.9383 & 316.0617 & & & & & & & $\mathrm{~L}, \mathrm{H}$ \\
\hline $1 \mathrm{~Hz}$ & 316.9382 & 317.0618 & & & & & & & $\mathrm{~L}, \mathrm{H}$ \\
\hline $1 \mathrm{~Hz}$ & 317.9381 & 318.0619 & & & & & & & $\mathrm{~L}, \mathrm{H}$ \\
\hline $1 \mathrm{~Hz}$ & 318.938 & 319.062 & & & & & & & $\mathrm{~L}, \mathrm{H}$ \\
\hline $1 \mathrm{~Hz}, 2 \mathrm{~Hz}$ & 319.9379 & 320.0621 & & & & & & & $\mathrm{~L}, \mathrm{H}$ \\
\hline SB & 320.3246 & 320.4488 & & & & & & & $\mathrm{H}$ \\
\hline $1 \mathrm{~Hz}$ & 320.9378 & 321.0622 & & & & & & & $\mathrm{~L}, \mathrm{H}$ \\
\hline $1 \mathrm{~Hz}$ & 321.9377 & 322.0623 & & & & & & & $\mathrm{~L}, \mathrm{H}$ \\
\hline $1 \mathrm{~Hz}$ & 322.9376 & 323.0624 & & & & & & & $\mathrm{~L}, \mathrm{H}$ \\
\hline $1 \mathrm{~Hz}$ & 323.9375 & 324.0625 & & & & & & & $\mathrm{~L}, \mathrm{H}$ \\
\hline $1 \mathrm{~Hz}$ & 324.9374 & 325.0626 & & & & & & & $\mathrm{~L}, \mathrm{H}$ \\
\hline $1 \mathrm{~Hz}$ & 325.9373 & 326.0627 & & & & & & & $\mathrm{~L}, \mathrm{H}$ \\
\hline $1 \mathrm{~Hz}$ & 326.9372 & 327.0628 & & & & & & & $\mathrm{~L}, \mathrm{H}$ \\
\hline $1 \mathrm{~Hz}$ & 327.9371 & 328.0629 & & & & & & & $\mathrm{~L}, \mathrm{H}$ \\
\hline $1 \mathrm{~Hz}$ & 328.937 & 329.063 & & & & & & & $\mathrm{~L}, \mathrm{H}$ \\
\hline VM & & & 329.1476 & 329.2724 & 329.2724 & 329.3676 & 329.3676 & 329.4924 & $\mathrm{~L}$ \\
\hline $1 \mathrm{~Hz}, \mathrm{E}, \mathrm{VM}$ & & & 329.3376 & 329.4624 & 329.4624 & 329.9381 & 329.9381 & 330.0631 & $\mathrm{H}$ \\
\hline $1 \mathrm{~Hz}$ & 329.9369 & 330.0631 & & & & & & & $\mathrm{~L}$ \\
\hline $1 \mathrm{~Hz}$ & 330.9368 & 331.0632 & & & & & & & $\mathrm{~L}, \mathrm{H}$ \\
\hline $1 \mathrm{~Hz}$ & 331.9367 & 332.0633 & & & & & & & $\mathrm{~L}, \mathrm{H}$ \\
\hline $1 \mathrm{~Hz}$ & 332.9366 & 333.0634 & & & & & & & $\mathrm{~L}, \mathrm{H}$ \\
\hline $1 \mathrm{~Hz}, 2 \mathrm{~Hz}$ & 333.9365 & 334.0635 & & & & & & & $\mathrm{~L}, \mathrm{H}$ \\
\hline $1 \mathrm{~Hz}$ & 334.9364 & 335.0636 & & & & & & & $\mathrm{~L}, \mathrm{H}$ \\
\hline VM & & & 335.187 & 335.313 & 335.313 & 335.747 & 335.747 & 335.873 & $\mathrm{~L}$ \\
\hline $1 \mathrm{~Hz}, \mathrm{VM}$ & & & 335.437 & 335.563 & 335.563 & 335.9375 & 335.9375 & 336.0637 & $\mathrm{H}$ \\
\hline $1 \mathrm{~Hz}$ & 335.9363 & 336.0637 & & & & & & & $\mathrm{~L}$ \\
\hline $1 \mathrm{~Hz}$ & 336.9362 & 337.0638 & & & & & & & $\mathrm{~L}, \mathrm{H}$ \\
\hline $1 \mathrm{~Hz}$ & 337.9361 & 338.0639 & & & & & & & $\mathrm{~L}, \mathrm{H}$ \\
\hline $1 \mathrm{~Hz}$ & 338.936 & 339.064 & & & & & & & $\mathrm{~L}, \mathrm{H}$ \\
\hline $1 \mathrm{~Hz}, \mathrm{VM}$ & & & 339.9359 & 340.0629 & 340.0629 & 349.9361 & 349.9361 & 350.0651 & $\mathrm{~L}$ \\
\hline $1 \mathrm{~Hz}$ & 339.9359 & 340.0641 & & & & & & & $\mathrm{H}$ \\
\hline $1 \mathrm{~Hz}$ & 340.9358 & 341.0642 & & & & & & & $\mathrm{H}$ \\
\hline $1 \mathrm{~Hz}, \mathrm{SB}, \mathrm{VM}$ & & & 341.9357 & 342.0631 & 342.0631 & 349.9361 & 349.9361 & 350.0651 & $\mathrm{H}$ \\
\hline $1 \mathrm{~Hz}$ & 350.9348 & 351.0652 & & & & & & & $\mathrm{~L}, \mathrm{H}$ \\
\hline $1 \mathrm{~Hz}$ & 351.9347 & 352.0653 & & & & & & & $\mathrm{~L}, \mathrm{H}$ \\
\hline $1 \mathrm{~Hz}$ & 352.9346 & 353.0654 & & & & & & & $\mathrm{~L}, \mathrm{H}$ \\
\hline $1 \mathrm{~Hz}$ & 353.9345 & 354.0655 & & & & & & & $\mathrm{~L}, \mathrm{H}$ \\
\hline $1 \mathrm{~Hz}$ & 354.9344 & 355.0656 & & & & & & & $\mathrm{~L}, \mathrm{H}$ \\
\hline $1 \mathrm{~Hz}$ & 355.9343 & 356.0657 & & & & & & & $\mathrm{~L}, \mathrm{H}$ \\
\hline $1 \mathrm{~Hz}$ & 356.9342 & 357.0658 & & & & & & & $\mathrm{~L}, \mathrm{H}$ \\
\hline $1 \mathrm{~Hz}$ & 357.9341 & 358.0659 & & & & & & & $\mathrm{~L}, \mathrm{H}$ \\
\hline $1 \mathrm{~Hz}, \mathrm{M}$ & & & 358.934 & 359.0648 & 359.0648 & 360.935 & 360.935 & 361.0662 & $\mathrm{~L}, \mathrm{H}$ \\
\hline $1 \mathrm{~Hz}$ & 361.9337 & 362.0663 & & & & & & & $\mathrm{~L}, \mathrm{H}$ \\
\hline $1 \mathrm{~Hz}$ & 362.9336 & 363.0664 & & & & & & & $\mathrm{~L}, \mathrm{H}$ \\
\hline $1 \mathrm{~Hz}$ & 363.9335 & 364.0665 & & & & & & & $\mathrm{~L}, \mathrm{H}$ \\
\hline $1 \mathrm{~Hz}$ & 364.9334 & 365.0666 & & & & & & & $\mathrm{~L}, \mathrm{H}$ \\
\hline $1 \mathrm{~Hz}$ & 365.9333 & 366.0667 & & & & & & & $\mathrm{~L}, \mathrm{H}$ \\
\hline $1 \mathrm{~Hz}$ & 366.9332 & 367.0668 & & & & & & & $\mathrm{~L}, \mathrm{H}$ \\
\hline $1 \mathrm{~Hz}$ & 367.9331 & 368.0669 & & & & & & & $\mathrm{~L}, \mathrm{H}$ \\
\hline
\end{tabular}


TABLE IV. (Continued)

\begin{tabular}{|c|c|c|c|c|c|c|}
\hline \multirow{2}{*}{$\frac{\text { Line type }}{1 \mathrm{~Hz}}$} & \multicolumn{2}{|c|}{ Mixed, isolated } & \multirow[t]{2}{*}{ Mixed, left } & \multirow[t]{2}{*}{ All fake data } & \multirow[t]{2}{*}{ Mixed,right } & \multirow{2}{*}{$\frac{\text { Detector }}{\mathrm{L}, \mathrm{H}}$} \\
\hline & 368.933 & 369.067 & & & & \\
\hline $1 \mathrm{~Hz}$ & 369.9329 & 370.0671 & & & & L,H \\
\hline $1 \mathrm{~Hz}$ & 370.9328 & 371.0672 & & & & $\mathrm{~L}, \mathrm{H}$ \\
\hline SB & 371.7405 & 371.8749 & & & & $\mathrm{H}$ \\
\hline $1 \mathrm{~Hz}, 2 \mathrm{~Hz}$ & 371.9327 & 372.0673 & & & & $\mathrm{~L}, \mathrm{H}$ \\
\hline $1 \mathrm{~Hz}$ & 372.9326 & 373.0674 & & & & $\mathrm{~L}, \mathrm{H}$ \\
\hline $\mathrm{D}$ & 373.3832 & 373.6168 & & & & $\mathrm{H}$ \\
\hline $1 \mathrm{~Hz}$ & 373.9325 & 374.0675 & & & & $\mathrm{~L}, \mathrm{H}$ \\
\hline $1 \mathrm{~Hz}$ & 374.9324 & 375.0676 & & & & $\mathrm{~L}, \mathrm{H}$ \\
\hline $1 \mathrm{~Hz}, 2 \mathrm{~Hz}$ & 375.9323 & 376.0677 & & & & $\mathrm{~L}, \mathrm{H}$ \\
\hline $1 \mathrm{~Hz}$ & 376.9322 & 377.0678 & & & & $\mathrm{~L}, \mathrm{H}$ \\
\hline $1 \mathrm{~Hz}$ & 377.9321 & 378.0679 & & & & $\mathrm{~L}, \mathrm{H}$ \\
\hline $1 \mathrm{~Hz}$ & 378.932 & 379.068 & & & & $\mathrm{~L}, \mathrm{H}$ \\
\hline $1 \mathrm{~Hz}, 2 \mathrm{~Hz}$ & 379.9319 & 380.0681 & & & & $\mathrm{~L}, \mathrm{H}$ \\
\hline $1 \mathrm{~Hz}$ & 380.9318 & 381.0682 & & & & $\mathrm{~L}, \mathrm{H}$ \\
\hline $1 \mathrm{~Hz}$ & 381.9317 & 382.0683 & & & & $\mathrm{~L}, \mathrm{H}$ \\
\hline $1 \mathrm{~Hz}$ & 382.9316 & 383.0684 & & & & $\mathrm{~L}, \mathrm{H}$ \\
\hline SB & 383.5955 & 383.7323 & & & & $\mathrm{H}$ \\
\hline $1 \mathrm{~Hz}, 2 \mathrm{~Hz}$ & 383.9315 & 384.0685 & & & & $\mathrm{~L}, \mathrm{H}$ \\
\hline $1 \mathrm{~Hz}$ & 384.9314 & 385.0686 & & & & $\mathrm{~L}, \mathrm{H}$ \\
\hline $1 \mathrm{~Hz}$ & 385.9313 & 386.0687 & & & & $\mathrm{~L}, \mathrm{H}$ \\
\hline $1 \mathrm{~Hz}$ & 386.9312 & 387.0688 & & & & $\mathrm{~L}, \mathrm{H}$ \\
\hline $1 \mathrm{~Hz}$ & 387.9311 & 388.0689 & & & & $\mathrm{~L}, \mathrm{H}$ \\
\hline $1 \mathrm{~Hz}$ & 388.931 & 389.069 & & & & $\mathrm{~L}, \mathrm{H}$ \\
\hline $1 \mathrm{~Hz}$ & 389.9309 & 390.0691 & & & & $\mathrm{~L}, \mathrm{H}$ \\
\hline $1 \mathrm{~Hz}$ & 390.9308 & 391.0692 & & & & $\mathrm{~L}, \mathrm{H}$ \\
\hline $1 \mathrm{~Hz}$ & 391.9307 & 392.0693 & & & & $\mathrm{~L}, \mathrm{H}$ \\
\hline $\mathrm{D}$ & 392.1307 & 392.2693 & & & & $\mathrm{~L}, \mathrm{H}$ \\
\hline $1 \mathrm{~Hz}$ & 392.9306 & 393.0694 & & & & $\mathrm{~L}, \mathrm{H}$ \\
\hline $1 \mathrm{~Hz}, 2 \mathrm{~Hz}$ & 393.9305 & 394.0695 & & & & $\mathrm{~L}, \mathrm{H}$ \\
\hline $1 \mathrm{~Hz}$ & 394.9304 & 395.0696 & & & & $\mathrm{~L}, \mathrm{H}$ \\
\hline $1 \mathrm{~Hz}$ & 395.9303 & 396.0697 & & & & $\mathrm{~L}, \mathrm{H}$ \\
\hline $1 \mathrm{~Hz}$ & 396.9302 & 397.0698 & & & & $\mathrm{~L}, \mathrm{H}$ \\
\hline SB & 397.4496 & 397.5892 & & & & $\mathrm{H}$ \\
\hline $1 \mathrm{~Hz}$ & 397.9301 & 398.0699 & & & & $\mathrm{~L}, \mathrm{H}$ \\
\hline $1 \mathrm{~Hz}$ & 398.93 & 399.07 & & & & $\mathrm{~L}, \mathrm{H}$ \\
\hline $\mathrm{D}$ & 399.23 & 399.37 & & & & $\mathrm{~L}$ \\
\hline $1 \mathrm{~Hz}$ & 399.9299 & 400.0701 & & & & $\mathrm{~L}, \mathrm{H}$ \\
\hline $1 \mathrm{~Hz}$ & 400.9298 & 401.0702 & & & & $\mathrm{~L}, \mathrm{H}$ \\
\hline $\mathrm{D}$ & 401.3804 & 401.6196 & & & & $\mathrm{H}$ \\
\hline $1 \mathrm{~Hz}, 2 \mathrm{~Hz}$ & 401.9297 & 402.0703 & & & & $\mathrm{~L}, \mathrm{H}$ \\
\hline $1 \mathrm{~Hz}$ & 402.9296 & 403.0704 & & & & $\mathrm{~L}, \mathrm{H}$ \\
\hline $1 \mathrm{~Hz}$ & 403.9295 & 404.0705 & & & & $\mathrm{~L}, \mathrm{H}$ \\
\hline $1 \mathrm{~Hz}$ & 404.9294 & 405.0706 & & & & $\mathrm{~L}, \mathrm{H}$ \\
\hline $1 \mathrm{~Hz}$ & 405.9293 & 406.0707 & & & & $\mathrm{~L}, \mathrm{H}$ \\
\hline $1 \mathrm{~Hz}$ & 406.9292 & 407.0708 & & & & $\mathrm{~L}, \mathrm{H}$ \\
\hline $1 \mathrm{~Hz}$ & 407.9291 & 408.0709 & & & & $\mathrm{~L}, \mathrm{H}$ \\
\hline $1 \mathrm{~Hz}$ & 408.929 & 409.071 & & & & $\mathrm{~L}, \mathrm{H}$ \\
\hline $\mathrm{SB}$ & 409.2995 & 409.4415 & & & & $\mathrm{H}$ \\
\hline $1 \mathrm{~Hz}, 2 \mathrm{~Hz}$ & 409.9289 & 410.0711 & & & & $\mathrm{~L}, \mathrm{H}$ \\
\hline $1 \mathrm{~Hz}$ & 410.9288 & 411.0712 & & & & $\mathrm{~L}, \mathrm{H}$ \\
\hline $1 \mathrm{~Hz}$ & 411.9287 & 412.0713 & & & & $\mathrm{~L}, \mathrm{H}$ \\
\hline $1 \mathrm{~Hz}$ & 412.9286 & 413.0714 & & & & $\mathrm{~L}, \mathrm{H}$ \\
\hline $1 \mathrm{~Hz}, 2 \mathrm{~Hz}$ & 413.9285 & 414.0715 & & & & $\mathrm{~L}, \mathrm{H}$ \\
\hline $1 \mathrm{~Hz}$ & 414.9284 & 415.0716 & & & & $\mathrm{~L}, \mathrm{H}$ \\
\hline
\end{tabular}


TABLE IV. (Continued)

\begin{tabular}{|c|c|c|c|c|c|c|c|c|c|}
\hline \multirow{2}{*}{$\frac{\text { Line type }}{1 \mathrm{~Hz}}$} & \multicolumn{2}{|c|}{ Mixed, isolated } & \multicolumn{2}{|c|}{ Mixed, left } & \multicolumn{2}{|c|}{ All fake data } & \multicolumn{2}{|c|}{ Mixed,right } & \multirow{2}{*}{$\frac{\text { Detector }}{\mathrm{L}, \mathrm{H}}$} \\
\hline & 415.9283 & 416.0717 & & & & & & & \\
\hline $1 \mathrm{~Hz}$ & 416.9282 & 417.0718 & & & & & & & $\mathrm{~L}, \mathrm{H}$ \\
\hline $1 \mathrm{~Hz}, 2 \mathrm{~Hz}$ & 417.9281 & 418.0719 & & & & & & & $\mathrm{~L}, \mathrm{H}$ \\
\hline $1 \mathrm{~Hz}, \mathrm{M}$ & & & 418.928 & 419.0708 & 419.0708 & 420.929 & 420.929 & 421.0722 & $\mathrm{~L}, \mathrm{H}$ \\
\hline $1 \mathrm{~Hz}, 2 \mathrm{~Hz}$ & 421.9277 & 422.0723 & & & & & & & $\mathrm{~L}, \mathrm{H}$ \\
\hline $1 \mathrm{~Hz}$ & 422.9276 & 423.0724 & & & & & & & $\mathrm{~L}, \mathrm{H}$ \\
\hline SB & 423.1582 & 423.303 & & & & & & & $\mathrm{H}$ \\
\hline $1 \mathrm{~Hz}$ & 423.9275 & 424.0725 & & & & & & & $\mathrm{~L}, \mathrm{H}$ \\
\hline $1 \mathrm{~Hz}$ & 424.9274 & 425.0726 & & & & & & & $\mathrm{~L}, \mathrm{H}$ \\
\hline $1 \mathrm{~Hz}$ & 425.9273 & 426.0727 & & & & & & & $\mathrm{~L}, \mathrm{H}$ \\
\hline $1 \mathrm{~Hz}$ & 426.9272 & 427.0728 & & & & & & & $\mathrm{~L}, \mathrm{H}$ \\
\hline $1 \mathrm{~Hz}$ & 427.9271 & 428.0729 & & & & & & & $\mathrm{~L}, \mathrm{H}$ \\
\hline $1 \mathrm{~Hz}$ & 428.927 & 429.073 & & & & & & & $\mathrm{~L}, \mathrm{H}$ \\
\hline $1 \mathrm{~Hz}, 2 \mathrm{~Hz}$ & 429.9269 & 430.0731 & & & & & & & $\mathrm{~L}, \mathrm{H}$ \\
\hline $1 \mathrm{~Hz}$ & 430.9268 & 431.0732 & & & & & & & $\mathrm{~L}, \mathrm{H}$ \\
\hline $1 \mathrm{~Hz}, 2 \mathrm{~Hz}$ & 431.9267 & 432.0733 & & & & & & & $\mathrm{~L}, \mathrm{H}$ \\
\hline $1 \mathrm{~Hz}$ & 432.9266 & 433.0734 & & & & & & & $\mathrm{~L}, \mathrm{H}$ \\
\hline $1 \mathrm{~Hz}$ & 433.9265 & 434.0735 & & & & & & & $\mathrm{~L}, \mathrm{H}$ \\
\hline $1 \mathrm{~Hz}, 2 \mathrm{~Hz}$ & 434.9264 & 435.0736 & & & & & & & $\mathrm{~L}, \mathrm{H}$ \\
\hline SB & 435.0125 & 435.1597 & & & & & & & $\mathrm{H}$ \\
\hline $1 \mathrm{~Hz}$ & 435.9263 & 436.0737 & & & & & & & $\mathrm{~L}, \mathrm{H}$ \\
\hline $1 \mathrm{~Hz}$ & 436.9262 & 437.0738 & & & & & & & $\mathrm{~L}, \mathrm{H}$ \\
\hline $1 \mathrm{~Hz}$ & 437.9261 & 438.0739 & & & & & & & $\mathrm{~L}, \mathrm{H}$ \\
\hline $1 \mathrm{~Hz}$ & 438.926 & 439.074 & & & & & & & $\mathrm{~L}, \mathrm{H}$ \\
\hline $1 \mathrm{~Hz}, 2 \mathrm{~Hz}$ & 439.9259 & 440.0741 & & & & & & & $\mathrm{~L}, \mathrm{H}$ \\
\hline $1 \mathrm{~Hz}$ & 440.9258 & 441.0742 & & & & & & & $\mathrm{~L}, \mathrm{H}$ \\
\hline $1 \mathrm{~Hz}$ & 441.9257 & 442.0743 & & & & & & & $\mathrm{~L}, \mathrm{H}$ \\
\hline $1 \mathrm{~Hz}$ & 442.9256 & 443.0744 & & & & & & & $\mathrm{~L}, \mathrm{H}$ \\
\hline $1 \mathrm{~Hz}$ & 443.9255 & 444.0745 & & & & & & & $\mathrm{~L}, \mathrm{H}$ \\
\hline $1 \mathrm{~Hz}$ & 444.9254 & 445.0746 & & & & & & & $\mathrm{~L}, \mathrm{H}$ \\
\hline $1 \mathrm{~Hz}$ & 445.9253 & 446.0747 & & & & & & & $\mathrm{~L}, \mathrm{H}$ \\
\hline $1 \mathrm{~Hz}$ & 446.9252 & 447.0748 & & & & & & & $\mathrm{~L}, \mathrm{H}$ \\
\hline $1 \mathrm{~Hz}, 2 \mathrm{~Hz}$ & 447.9251 & 448.0749 & & & & & & & $\mathrm{~L}, \mathrm{H}$ \\
\hline $1 \mathrm{~Hz}$ & 448.925 & 449.075 & & & & & & & $\mathrm{~L}, \mathrm{H}$ \\
\hline $1 \mathrm{~Hz}$ & 449.9249 & 450.0751 & & & & & & & $\mathrm{~L}, \mathrm{H}$ \\
\hline $1 \mathrm{~Hz}$ & 450.9248 & 451.0752 & & & & & & & $\mathrm{~L}, \mathrm{H}$ \\
\hline $1 \mathrm{~Hz}$ & 451.9247 & 452.0753 & & & & & & & $\mathrm{~L}, \mathrm{H}$ \\
\hline $1 \mathrm{~Hz}$ & 452.9246 & 453.0754 & & & & & & & $\mathrm{~L}, \mathrm{H}$ \\
\hline $1 \mathrm{~Hz}$ & 453.9245 & 454.0755 & & & & & & & $\mathrm{~L}, \mathrm{H}$ \\
\hline $1 \mathrm{~Hz}$ & 454.9244 & 455.0756 & & & & & & & $\mathrm{~L}, \mathrm{H}$ \\
\hline $1 \mathrm{~Hz}$ & 455.9243 & 456.0757 & & & & & & & $\mathrm{~L}, \mathrm{H}$ \\
\hline $1 \mathrm{~Hz}$ & 456.9242 & 457.0758 & & & & & & & $\mathrm{~L}, \mathrm{H}$ \\
\hline $1 \mathrm{~Hz}$ & 457.9241 & 458.0759 & & & & & & & $\mathrm{~L}, \mathrm{H}$ \\
\hline $1 \mathrm{~Hz}$ & 458.924 & 459.076 & & & & & & & $\mathrm{~L}, \mathrm{H}$ \\
\hline $1 \mathrm{~Hz}$ & 459.9239 & 460.0761 & & & & & & & $\mathrm{~L}, \mathrm{H}$ \\
\hline SB & 460.7206 & 460.8728 & & & & & & & $\mathrm{H}$ \\
\hline $1 \mathrm{~Hz}$ & 460.9238 & 461.0762 & & & & & & & $\mathrm{~L}, \mathrm{H}$ \\
\hline $1 \mathrm{~Hz}, 2 \mathrm{~Hz}$ & 461.9237 & 462.0763 & & & & & & & $\mathrm{~L}, \mathrm{H}$ \\
\hline $1 \mathrm{~Hz}$ & 462.9236 & 463.0764 & & & & & & & $\mathrm{~L}, \mathrm{H}$ \\
\hline $1 \mathrm{~Hz}$ & 463.9235 & 464.0765 & & & & & & & $\mathrm{~L}, \mathrm{H}$ \\
\hline $1 \mathrm{~Hz}$ & 464.9234 & 465.0766 & & & & & & & $\mathrm{~L}, \mathrm{H}$ \\
\hline $1 \mathrm{~Hz}, 2 \mathrm{~Hz}$ & 465.9233 & 466.0767 & & & & & & & $\mathrm{~L}, \mathrm{H}$ \\
\hline $1 \mathrm{~Hz}$ & 466.9232 & 467.0768 & & & & & & & $\mathrm{~L}, \mathrm{H}$ \\
\hline $1 \mathrm{~Hz}, 2 \mathrm{~Hz}$ & 467.9231 & 468.0769 & & & & & & & $\mathrm{~L}, \mathrm{H}$ \\
\hline $1 \mathrm{~Hz}$ & 468.923 & 469.077 & & & & & & & $\mathrm{~L}, \mathrm{H}$ \\
\hline
\end{tabular}


TABLE IV. (Continued)

\begin{tabular}{|c|c|c|c|c|c|c|c|c|c|}
\hline \multirow{2}{*}{$\frac{\text { Line type }}{1 \mathrm{~Hz}, 2 \mathrm{~Hz}}$} & \multicolumn{2}{|c|}{ Mixed, isolated } & \multicolumn{2}{|c|}{ Mixed, left } & \multicolumn{2}{|c|}{ All fake data } & \multicolumn{2}{|c|}{ Mixed,right } & \multirow{2}{*}{$\frac{\text { Detector }}{\mathrm{L}, \mathrm{H}}$} \\
\hline & 469.9229 & 470.0771 & & & & & & & \\
\hline $1 \mathrm{~Hz}$ & 470.9228 & 471.0772 & & & & & & & $\mathrm{~L}, \mathrm{H}$ \\
\hline $1 \mathrm{~Hz}$ & 471.9227 & 472.0773 & & & & & & & $\mathrm{~L}, \mathrm{H}$ \\
\hline $1 \mathrm{~Hz}$ & 472.9226 & 473.0774 & & & & & & & $\mathrm{~L}, \mathrm{H}$ \\
\hline $1 \mathrm{~Hz}, 2 \mathrm{~Hz}$ & 473.9225 & 474.0775 & & & & & & & $\mathrm{~L}, \mathrm{H}$ \\
\hline $1 \mathrm{~Hz}$ & 474.9224 & 475.0776 & & & & & & & $\mathrm{~L}, \mathrm{H}$ \\
\hline $1 \mathrm{~Hz}$ & 475.9223 & 476.0777 & & & & & & & $\mathrm{~L}, \mathrm{H}$ \\
\hline $1 \mathrm{~Hz}$ & 476.9222 & 477.0778 & & & & & & & $\mathrm{~L}, \mathrm{H}$ \\
\hline $1 \mathrm{~Hz}$ & 477.9221 & 478.0779 & & & & & & & $\mathrm{~L}, \mathrm{H}$ \\
\hline $1 \mathrm{~Hz}, \mathrm{M}$ & & & 478.922 & 479.0768 & 479.0768 & 480.923 & 480.923 & 481.0782 & $\mathrm{~L}, \mathrm{H}$ \\
\hline $1 \mathrm{~Hz}, 2 \mathrm{~Hz}$ & 481.9217 & 482.0783 & & & & & & & $\mathrm{~L}, \mathrm{H}$ \\
\hline $1 \mathrm{~Hz}$ & 482.9216 & 483.0784 & & & & & & & $\mathrm{~L}, \mathrm{H}$ \\
\hline $1 \mathrm{~Hz}$ & 483.9215 & 484.0785 & & & & & & & $\mathrm{~L}, \mathrm{H}$ \\
\hline $1 \mathrm{~Hz}$ & 484.9214 & 485.0786 & & & & & & & $\mathrm{~L}, \mathrm{H}$ \\
\hline $1 \mathrm{~Hz}$ & 485.9213 & 486.0787 & & & & & & & $\mathrm{~L}, \mathrm{H}$ \\
\hline SB & 486.4291 & 486.5865 & & & & & & & $\mathrm{H}$ \\
\hline $1 \mathrm{~Hz}$ & 486.9212 & 487.0788 & & & & & & & $\mathrm{~L}, \mathrm{H}$ \\
\hline $1 \mathrm{~Hz}, 2 \mathrm{~Hz}$ & 487.9211 & 488.0789 & & & & & & & $\mathrm{~L}, \mathrm{H}$ \\
\hline $1 \mathrm{~Hz}$ & 488.921 & 489.079 & & & & & & & $\mathrm{~L}, \mathrm{H}$ \\
\hline $1 \mathrm{~Hz}$ & 489.9209 & 490.0791 & & & & & & & $\mathrm{~L}, \mathrm{H}$ \\
\hline $1 \mathrm{~Hz}$ & 490.9208 & 491.0792 & & & & & & & $\mathrm{~L}, \mathrm{H}$ \\
\hline $1 \mathrm{~Hz}$ & 491.9207 & 492.0793 & & & & & & & $\mathrm{~L}, \mathrm{H}$ \\
\hline $1 \mathrm{~Hz}$ & 492.9206 & 493.0794 & & & & & & & $\mathrm{~L}, \mathrm{H}$ \\
\hline $1 \mathrm{~Hz}$ & 493.9205 & 494.0795 & & & & & & & $\mathrm{~L}, \mathrm{H}$ \\
\hline $1 \mathrm{~Hz}$ & 494.9204 & 495.0796 & & & & & & & $\mathrm{~L}, \mathrm{H}$ \\
\hline $1 \mathrm{~Hz}, 2 \mathrm{~Hz}$ & 495.9203 & 496.0797 & & & & & & & $\mathrm{~L}, \mathrm{H}$ \\
\hline $1 \mathrm{~Hz}$ & 496.9202 & 497.0798 & & & & & & & $\mathrm{~L}, \mathrm{H}$ \\
\hline $1 \mathrm{~Hz}$ & 497.9201 & 498.0799 & & & & & & & $\mathrm{~L}, \mathrm{H}$ \\
\hline $1 \mathrm{~Hz}$ & 498.92 & 499.08 & & & & & & & $\mathrm{~L}, \mathrm{H}$ \\
\hline $1 \mathrm{~Hz}, 2 \mathrm{~Hz}$ & 499.9199 & 500.0801 & & & & & & & $\mathrm{~L}, \mathrm{H}$ \\
\hline $1 \mathrm{~Hz}$ & 500.9198 & 501.0802 & & & & & & & $\mathrm{~L}, \mathrm{H}$ \\
\hline $1 \mathrm{~Hz}$ & 501.9197 & 502.0803 & & & & & & & $\mathrm{~L}, \mathrm{H}$ \\
\hline $1 \mathrm{~Hz}$ & 502.9196 & 503.0804 & & & & & & & $\mathrm{~L}, \mathrm{H}$ \\
\hline $1 \mathrm{~Hz}, 2 \mathrm{~Hz}$ & 503.9195 & 504.0805 & & & & & & & $\mathrm{~L}, \mathrm{H}$ \\
\hline $1 \mathrm{~Hz}$ & 504.9194 & 505.0806 & & & & & & & $\mathrm{~L}, \mathrm{H}$ \\
\hline $1 \mathrm{~Hz}$ & 505.9193 & 506.0807 & & & & & & & $\mathrm{~L}, \mathrm{H}$ \\
\hline $1 \mathrm{~Hz}$ & 506.9192 & 507.0808 & & & & & & & $\mathrm{~L}, \mathrm{H}$ \\
\hline $1 \mathrm{~Hz}, 2 \mathrm{~Hz}$ & 507.9191 & 508.0809 & & & & & & & $\mathrm{~L}, \mathrm{H}$ \\
\hline $1 \mathrm{~Hz}$ & 508.919 & 509.081 & & & & & & & $\mathrm{~L}, \mathrm{H}$ \\
\hline
\end{tabular}




\section{Signal-frequency ranges where the results might have contributions from fake data}

TABLE V. 50-mHz search-frequency bands that were identified as disturbed based on visual inspection (D) or where the results were produced from entirely fake data as detailed in Table I (C). Both sets of bands (D and C) were excluded from the analysis. The first two columns list the first frequency of the first and last $50-\mathrm{mHz}$ band in a contiguous range of excluded bands.

\begin{tabular}{|c|c|c|}
\hline Start band & Stop band & Disturbance type \\
\hline 50.113 & & $\mathrm{D}$ \\
\hline 50.563 & & $\mathrm{D}$ \\
\hline 51.013 & & $\mathrm{D}$ \\
\hline 51.113 & 51.163 & $\mathrm{D}$ \\
\hline 51.963 & & $\mathrm{D}$ \\
\hline 52.413 & 52.463 & $\mathrm{D}$ \\
\hline 52.613 & & $\mathrm{D}$ \\
\hline 52.863 & 53.113 & $\mathrm{D}$ \\
\hline 53.663 & & $\mathrm{D}$ \\
\hline 53.863 & 53.913 & $\mathrm{D}$ \\
\hline 54.363 & 54.513 & $\mathrm{D}$ \\
\hline 55.063 & 55.113 & $\mathrm{D}$ \\
\hline 55.463 & 55.613 & $\mathrm{D}$ \\
\hline 55.713 & 55.963 & $\mathrm{D}$ \\
\hline 56.113 & 56.513 & $\mathrm{D}$ \\
\hline 56.613 & & $\mathrm{D}$ \\
\hline 57.563 & & $\mathrm{D}$ \\
\hline 58.163 & & $\mathrm{D}$ \\
\hline 58.363 & 58.613 & $\mathrm{D}$ \\
\hline 58.713 & 59.013 & $\mathrm{D}$ \\
\hline 59.063 & & $\mathrm{C}$ \\
\hline 59.113 & & C D \\
\hline 59.163 & 60.863 & $\mathrm{C}$ \\
\hline 60.913 & & C D \\
\hline 60.963 & 61.013 & $\mathrm{D}$ \\
\hline 61.313 & 61.613 & $\mathrm{D}$ \\
\hline 61.963 & & $\mathrm{D}$ \\
\hline 62.213 & 62.263 & $\mathrm{D}$ \\
\hline 62.363 & & $\mathrm{D}$ \\
\hline 63.163 & 63.213 & $\mathrm{D}$ \\
\hline 63.463 & 63.513 & $\mathrm{D}$ \\
\hline 63.713 & & $\mathrm{D}$ \\
\hline 64.013 & 64.113 & $\mathrm{D}$ \\
\hline 64.313 & 64.513 & $\mathrm{D}$ \\
\hline 64.713 & 64.813 & $\mathrm{D}$ \\
\hline 65.313 & 65.413 & $\mathrm{D}$ \\
\hline 68.513 & & $\mathrm{D}$ \\
\hline 68.763 & 68.913 & $\mathrm{D}$ \\
\hline 69.263 & & $\mathrm{D}$ \\
\hline 69.713 & 69.763 & $\mathrm{D}$ \\
\hline 70.063 & 70.113 & $\mathrm{D}$ \\
\hline 70.463 & 70.513 & $\mathrm{D}$ \\
\hline 71.063 & & $\mathrm{D}$ \\
\hline 71.513 & & $\mathrm{D}$ \\
\hline 72.013 & 72.113 & $\mathrm{D}$ \\
\hline 72.313 & 72.413 & $\mathrm{D}$ \\
\hline 72.913 & 73.013 & $\mathrm{D}$ \\
\hline
\end{tabular}

(Table continued)
TABLE V. (Continued)

\begin{tabular}{|c|c|c|}
\hline Start band & Stop band & Disturbance type \\
\hline 73.313 & 73.413 & $\mathrm{D}$ \\
\hline 73.763 & 73.863 & $\mathrm{D}$ \\
\hline 74.463 & 74.513 & $\mathrm{D}$ \\
\hline 74.713 & & $\mathrm{D}$ \\
\hline 75.763 & & $\mathrm{D}$ \\
\hline 76.013 & 76.113 & $\mathrm{D}$ \\
\hline 77.563 & 77.663 & $\mathrm{D}$ \\
\hline 78.213 & 78.313 & $\mathrm{D}$ \\
\hline 78.413 & 78.563 & $\mathrm{D}$ \\
\hline 78.963 & 79.013 & $\mathrm{D}$ \\
\hline 79.263 & & $\mathrm{D}$ \\
\hline 79.913 & 80.063 & $\mathrm{D}$ \\
\hline 80.413 & 80.463 & $\mathrm{D}$ \\
\hline 80.563 & 80.663 & $\mathrm{D}$ \\
\hline 80.913 & & $\mathrm{D}$ \\
\hline 81.013 & 81.213 & $\mathrm{D}$ \\
\hline 83.463 & & $\mathrm{D}$ \\
\hline 83.863 & & $\mathrm{D}$ \\
\hline 85.413 & & $\mathrm{D}$ \\
\hline 85.563 & & $\mathrm{D}$ \\
\hline 85.713 & 85.813 & $\mathrm{D}$ \\
\hline 85.963 & & $\mathrm{D}$ \\
\hline 86.513 & & $\mathrm{D}$ \\
\hline 86.713 & 86.813 & $\mathrm{D}$ \\
\hline 87.763 & & $\mathrm{D}$ \\
\hline 88.513 & & $\mathrm{D}$ \\
\hline 88.963 & & $\mathrm{D}$ \\
\hline 89.963 & 90.013 & $\mathrm{D}$ \\
\hline 90.563 & 90.713 & $\mathrm{D}$ \\
\hline 91.513 & & $\mathrm{D}$ \\
\hline 92.113 & & $\mathrm{D}$ \\
\hline 93.063 & 93.113 & $\mathrm{D}$ \\
\hline 93.913 & 94.013 & $\mathrm{D}$ \\
\hline 94.213 & & $\mathrm{D}$ \\
\hline 96.663 & 97.163 & $\mathrm{D}$ \\
\hline 98.263 & & $\mathrm{D}$ \\
\hline 98.863 & 99.063 & $\mathrm{D}$ \\
\hline 99.713 & 99.763 & $\mathrm{D}$ \\
\hline 99.863 & 100.013 & $\mathrm{D}$ \\
\hline 100.213 & 100.263 & $\mathrm{D}$ \\
\hline 100.463 & & $\mathrm{D}$ \\
\hline 102.163 & 102.263 & $\mathrm{D}$ \\
\hline 102.663 & & $\mathrm{D}$ \\
\hline 103.013 & 103.113 & $\mathrm{D}$ \\
\hline 103.413 & 103.463 & $\mathrm{D}$ \\
\hline 104.863 & & $\mathrm{D}$ \\
\hline 105.663 & 105.713 & $\mathrm{D}$ \\
\hline 106.413 & 106.663 & $\mathrm{D}$ \\
\hline 107.013 & 107.213 & D \\
\hline 107.313 & 107.413 & $\mathrm{D}$ \\
\hline 107.663 & & $\mathrm{D}$ \\
\hline 108.013 & 108.063 & $\mathrm{D}$ \\
\hline 108.813 & 109.113 & $\mathrm{D}$ \\
\hline 109.413 & 109.513 & $\mathrm{D}$ \\
\hline 109.963 & & $\mathrm{D}$ \\
\hline
\end{tabular}

(Table continued) 
TABLE V. (Continued)

\begin{tabular}{|c|c|c|}
\hline Start band & Stop band & Disturbance type \\
\hline 110.613 & & $\mathrm{D}$ \\
\hline 110.863 & 110.913 & $\mathrm{D}$ \\
\hline 111.013 & 111.113 & $\mathrm{D}$ \\
\hline 111.713 & 111.863 & $\mathrm{D}$ \\
\hline 112.663 & 112.713 & $\mathrm{D}$ \\
\hline 113.063 & & $\mathrm{D}$ \\
\hline 113.213 & 113.313 & $\mathrm{D}$ \\
\hline 113.713 & 113.913 & $\mathrm{D}$ \\
\hline 114.663 & 114.713 & $\mathrm{D}$ \\
\hline 115.313 & 115.413 & $\mathrm{D}$ \\
\hline 115.763 & & $\mathrm{D}$ \\
\hline 117.463 & 117.713 & $\mathrm{D}$ \\
\hline 118.063 & & $\mathrm{D}$ \\
\hline 118.213 & 118.563 & $\mathrm{D}$ \\
\hline 118.813 & 119.013 & $\mathrm{D}$ \\
\hline 119.063 & 120.863 & $\mathrm{C}$ \\
\hline 120.963 & 121.013 & $\mathrm{D}$ \\
\hline 121.163 & 121.313 & $\mathrm{D}$ \\
\hline 121.613 & 121.763 & $\mathrm{D}$ \\
\hline 122.063 & 122.313 & $\mathrm{D}$ \\
\hline 123.113 & & $\mathrm{D}$ \\
\hline 125.613 & 125.663 & $\mathrm{D}$ \\
\hline 126.213 & 126.313 & $\mathrm{D}$ \\
\hline 126.413 & & $\mathrm{D}$ \\
\hline 126.713 & 126.813 & $\mathrm{D}$ \\
\hline 127.063 & & $\mathrm{D}$ \\
\hline 127.963 & 128.013 & $\mathrm{D}$ \\
\hline 128.363 & & $\mathrm{D}$ \\
\hline 129.713 & 129.763 & $\mathrm{D}$ \\
\hline 129.863 & 129.963 & $\mathrm{D}$ \\
\hline 130.513 & & $\mathrm{D}$ \\
\hline 131.263 & & $\mathrm{D}$ \\
\hline 132.713 & 132.763 & $\mathrm{D}$ \\
\hline 133.413 & & $\mathrm{D}$ \\
\hline 134.013 & 134.063 & $\mathrm{D}$ \\
\hline 134.413 & 134.513 & $\mathrm{D}$ \\
\hline 135.063 & 135.163 & $\mathrm{D}$ \\
\hline 135.613 & 135.713 & $\mathrm{D}$ \\
\hline 137.063 & 137.113 & $\mathrm{D}$ \\
\hline 137.463 & 137.513 & $\mathrm{D}$ \\
\hline 137.613 & 137.913 & $\mathrm{D}$ \\
\hline 138.163 & & $\mathrm{D}$ \\
\hline 139.463 & 139.513 & $\mathrm{D}$ \\
\hline 139.613 & 139.813 & $\mathrm{D}$ \\
\hline 140.113 & 140.213 & $\mathrm{D}$ \\
\hline 140.363 & 140.413 & $\mathrm{D}$ \\
\hline 140.963 & & $\mathrm{D}$ \\
\hline 141.613 & 141.663 & $\mathrm{D}$ \\
\hline 141.813 & 141.863 & $\mathrm{D}$ \\
\hline 142.213 & 142.313 & $\mathrm{D}$ \\
\hline 142.613 & 142.713 & $\mathrm{D}$ \\
\hline 144.313 & 144.363 & $\mathrm{D}$ \\
\hline 144.613 & 144.763 & $\mathrm{D}$ \\
\hline 145.013 & 145.713 & $\mathrm{D}$ \\
\hline 146.313 & 146.363 & $\mathrm{D}$ \\
\hline
\end{tabular}

(Table continued)
TABLE V. (Continued)

\begin{tabular}{|c|c|c|}
\hline Start band & Stop band & Disturbance type \\
\hline 146.663 & 146.813 & D \\
\hline 146.913 & & $\mathrm{D}$ \\
\hline 147.113 & 147.163 & $\mathrm{D}$ \\
\hline 147.713 & & $\mathrm{D}$ \\
\hline 148.763 & & $\mathrm{D}$ \\
\hline 149.113 & 149.213 & D \\
\hline 149.563 & & $\mathrm{D}$ \\
\hline 149.963 & 150.013 & D \\
\hline 150.863 & & $\mathrm{D}$ \\
\hline 151.263 & & $\mathrm{D}$ \\
\hline 152.063 & & $\mathrm{D}$ \\
\hline 153.163 & 153.313 & $\mathrm{D}$ \\
\hline 153.413 & & $\mathrm{D}$ \\
\hline 153.613 & 153.713 & $\mathrm{D}$ \\
\hline 153.863 & 153.913 & $\mathrm{D}$ \\
\hline 154.213 & 154.263 & $\mathrm{D}$ \\
\hline 155.263 & & $\mathrm{D}$ \\
\hline 155.763 & & $\mathrm{D}$ \\
\hline 156.113 & & $\mathrm{D}$ \\
\hline 156.213 & 156.263 & $\mathrm{D}$ \\
\hline 156.363 & & $\mathrm{D}$ \\
\hline 156.513 & & $\mathrm{D}$ \\
\hline 156.813 & 156.863 & $\mathrm{D}$ \\
\hline 157.363 & 157.413 & $\mathrm{D}$ \\
\hline 157.763 & & $\mathrm{D}$ \\
\hline 158.163 & & $\mathrm{D}$ \\
\hline 158.363 & & $\mathrm{D}$ \\
\hline 160.213 & 160.313 & $\mathrm{D}$ \\
\hline 161.413 & 161.513 & $\mathrm{D}$ \\
\hline 162.313 & 162.363 & $\mathrm{D}$ \\
\hline 162.913 & 162.963 & $\mathrm{D}$ \\
\hline 163.463 & 163.513 & D \\
\hline 168.063 & 168.113 & $\mathrm{D}$ \\
\hline 169.613 & 169.713 & D \\
\hline 170.813 & & $\mathrm{D}$ \\
\hline 173.713 & 173.813 & D \\
\hline 174.163 & & D \\
\hline 178.513 & & D \\
\hline 178.963 & & $\mathrm{D}$ \\
\hline 179.063 & 180.863 & $\mathrm{C}$ \\
\hline 180.963 & 181.013 & $\mathrm{D}$ \\
\hline 181.363 & & $\mathrm{D}$ \\
\hline 181.813 & 181.863 & D \\
\hline 182.763 & 182.813 & $\mathrm{D}$ \\
\hline 184.363 & 184.463 & $\mathrm{D}$ \\
\hline 185.363 & 185.413 & $\mathrm{D}$ \\
\hline 187.963 & & $\mathrm{D}$ \\
\hline 188.463 & & $\mathrm{D}$ \\
\hline 189.363 & 189.413 & $\mathrm{D}$ \\
\hline 189.813 & 189.863 & $\mathrm{D}$ \\
\hline 190.763 & 190.813 & $\mathrm{D}$ \\
\hline 192.363 & 192.613 & $\mathrm{D}$ \\
\hline 193.613 & 194.313 & $\mathrm{D}$ \\
\hline 196.963 & 197.013 & $\mathrm{D}$ \\
\hline 197.713 & 197.763 & D \\
\hline
\end{tabular}

(Table continued) 
TABLE V. (Continued)

\begin{tabular}{|c|c|c|}
\hline Start band & Stop band & Disturbance type \\
\hline 197.863 & 197.963 & $\mathrm{D}$ \\
\hline 198.113 & 198.163 & $\mathrm{D}$ \\
\hline 198.563 & 198.663 & $\mathrm{D}$ \\
\hline 199.213 & 199.313 & $\mathrm{D}$ \\
\hline 199.513 & 199.613 & $\mathrm{D}$ \\
\hline 199.813 & 200.013 & $\mathrm{D}$ \\
\hline 200.413 & 200.513 & $\mathrm{D}$ \\
\hline 200.913 & 201.013 & $\mathrm{D}$ \\
\hline 201.313 & 201.363 & $\mathrm{D}$ \\
\hline 203.663 & & $\mathrm{D}$ \\
\hline 204.113 & 204.213 & $\mathrm{D}$ \\
\hline 204.863 & 204.913 & $\mathrm{D}$ \\
\hline 205.663 & & $\mathrm{D}$ \\
\hline 205.863 & & $\mathrm{D}$ \\
\hline 206.413 & 206.663 & $\mathrm{D}$ \\
\hline 209.213 & 209.263 & $\mathrm{D}$ \\
\hline 210.213 & 210.313 & $\mathrm{D}$ \\
\hline 215.513 & & $\mathrm{D}$ \\
\hline 217.463 & 217.563 & $\mathrm{D}$ \\
\hline 217.913 & 218.013 & $\mathrm{D}$ \\
\hline 223.563 & 223.613 & $\mathrm{D}$ \\
\hline 225.513 & 225.563 & $\mathrm{D}$ \\
\hline 229.363 & & $\mathrm{D}$ \\
\hline 229.813 & 229.913 & $\mathrm{D}$ \\
\hline 230.213 & 230.313 & $\mathrm{D}$ \\
\hline 231.313 & 231.413 & $\mathrm{D}$ \\
\hline 234.113 & 234.163 & $\mathrm{D}$ \\
\hline 239.063 & 240.863 & $\mathrm{C}$ \\
\hline 241.613 & 241.713 & $\mathrm{D}$ \\
\hline 241.813 & 241.863 & $\mathrm{D}$ \\
\hline 242.113 & 242.163 & $\mathrm{D}$ \\
\hline 242.313 & 242.863 & $\mathrm{D}$ \\
\hline 246.513 & 246.563 & $\mathrm{D}$ \\
\hline 249.963 & 250.013 & $\mathrm{D}$ \\
\hline 253.063 & 253.113 & $\mathrm{D}$ \\
\hline 255.063 & 255.113 & $\mathrm{D}$ \\
\hline 257.063 & 257.113 & $\mathrm{D}$ \\
\hline 257.263 & 257.363 & $\mathrm{D}$ \\
\hline 259.063 & 259.113 & $\mathrm{D}$ \\
\hline 270.163 & 270.213 & $\mathrm{D}$ \\
\hline 270.613 & 270.663 & $\mathrm{D}$ \\
\hline 272.413 & 272.513 & $\mathrm{D}$ \\
\hline 275.213 & 275.563 & $\mathrm{D}$ \\
\hline 279.763 & 279.813 & $\mathrm{D}$ \\
\hline 280.313 & 280.363 & $\mathrm{D}$ \\
\hline 280.813 & & $\mathrm{D}$ \\
\hline 281.913 & 282.013 & $\mathrm{D}$ \\
\hline 289.363 & & $\mathrm{D}$ \\
\hline 290.963 & 291.013 & $\mathrm{D}$ \\
\hline 291.213 & & $\mathrm{D}$ \\
\hline 291.313 & 291.413 & $\mathrm{D}$ \\
\hline 299.063 & 300.863 & $\mathrm{C}$ \\
\hline 306.463 & 306.563 & $\mathrm{D}$ \\
\hline 308.463 & 308.513 & $\mathrm{D}$ \\
\hline 324.013 & 324.063 & $\mathrm{D}$ \\
\hline
\end{tabular}

(Table continued)
TABLE V. (Continued)

\begin{tabular}{|c|c|c|}
\hline Start band & Stop band & Disturbance type \\
\hline 329.113 & 329.263 & $\mathrm{D}$ \\
\hline 329.313 & & $\mathrm{C}$ \\
\hline 329.463 & 329.863 & $\mathrm{C}$ \\
\hline 332.163 & 332.263 & $\mathrm{D}$ \\
\hline 335.163 & 335.263 & $\mathrm{D}$ \\
\hline 335.313 & 335.663 & $\mathrm{C}$ \\
\hline 335.713 & & C D \\
\hline 335.763 & & C D \\
\hline 335.813 & & C D \\
\hline 335.863 & & $\mathrm{C}$ \\
\hline 338.613 & 338.863 & $\mathrm{D}$ \\
\hline 339.013 & 339.213 & $\mathrm{D}$ \\
\hline 339.463 & 340.013 & $\mathrm{D}$ \\
\hline 340.063 & & C D \\
\hline 340.113 & 349.863 & $\mathrm{C}$ \\
\hline 349.963 & 350.563 & $\mathrm{D}$ \\
\hline 350.663 & 350.763 & $\mathrm{D}$ \\
\hline 350.913 & 351.213 & $\mathrm{D}$ \\
\hline 351.363 & 351.563 & $\mathrm{D}$ \\
\hline 357.863 & 357.963 & $\mathrm{D}$ \\
\hline 358.913 & 359.063 & $\mathrm{D}$ \\
\hline 359.113 & 360.863 & $\mathrm{C}$ \\
\hline 360.913 & 361.013 & $\mathrm{D}$ \\
\hline 365.563 & 365.613 & $\mathrm{D}$ \\
\hline 366.513 & 366.613 & $\mathrm{D}$ \\
\hline 366.863 & & $\mathrm{D}$ \\
\hline 369.763 & 369.813 & $\mathrm{D}$ \\
\hline 370.013 & 370.163 & $\mathrm{D}$ \\
\hline 373.263 & 373.313 & $\mathrm{D}$ \\
\hline 373.513 & 373.613 & $\mathrm{D}$ \\
\hline 374.163 & & $\mathrm{D}$ \\
\hline 374.513 & 374.613 & $\mathrm{D}$ \\
\hline 389.513 & 389.613 & $\mathrm{D}$ \\
\hline 391.213 & 391.313 & $\mathrm{D}$ \\
\hline 392.063 & 392.213 & $\mathrm{D}$ \\
\hline 392.513 & 392.613 & $\mathrm{D}$ \\
\hline 393.013 & 393.263 & $\mathrm{D}$ \\
\hline 393.413 & 393.513 & $\mathrm{D}$ \\
\hline 393.913 & & $\mathrm{D}$ \\
\hline 394.013 & 394.213 & $\mathrm{D}$ \\
\hline 394.813 & 394.913 & $\mathrm{D}$ \\
\hline 395.463 & 395.513 & $\mathrm{D}$ \\
\hline 395.863 & & $\mathrm{D}$ \\
\hline 395.963 & 396.063 & $\mathrm{D}$ \\
\hline 396.513 & 396.713 & $\mathrm{D}$ \\
\hline 399.063 & 399.113 & $\mathrm{D}$ \\
\hline 399.213 & 399.313 & $\mathrm{D}$ \\
\hline 399.613 & 400.613 & $\mathrm{D}$ \\
\hline 400.713 & 400.813 & $\mathrm{D}$ \\
\hline 401.013 & 401.313 & $\mathrm{D}$ \\
\hline 403.513 & 403.913 & $\mathrm{D}$ \\
\hline 407.213 & 407.363 & $\mathrm{D}$ \\
\hline 407.813 & 407.913 & $\mathrm{D}$ \\
\hline 408.213 & 408.363 & $\mathrm{D}$ \\
\hline 409.263 & 409.413 & $\mathrm{D}$ \\
\hline
\end{tabular}

(Table continued) 
TABLE V. (Continued)

\begin{tabular}{llc}
\hline \hline Start band & Stop band & Disturbance type \\
\hline 413.013 & 413.363 & $\mathrm{D}$ \\
416.113 & 416.213 & $\mathrm{D}$ \\
417.013 & 417.213 & $\mathrm{D}$ \\
418.163 & 418.213 & $\mathrm{D}$ \\
419.113 & 420.863 & $\mathrm{C}$ \\
421.213 & & $\mathrm{D}$ \\
\hline
\end{tabular}

(Table continued)
TABLE V. (Continued)

\begin{tabular}{llc}
\hline \hline Start band & Stop band & Disturbance type \\
\hline 430.313 & & $\mathrm{D}$ \\
444.413 & 444.513 & $\mathrm{D}$ \\
448.913 & & $\mathrm{D}$ \\
451.413 & 451.663 & $\mathrm{D}$ \\
472.563 & 472.663 & $\mathrm{D}$ \\
474.563 & 474.613 & $\mathrm{D}$ \\
479.113 & 480.863 & $\mathrm{C}$ \\
\hline \hline
\end{tabular}

[1] https://www.einsteinathome.org/.

[2] B. P. Abbott et al. (LIGO Scientific and Virgo Collaborations), Comprehensive all-sky search for periodic gravitational waves in the sixth science run LIGO data, Phys. Rev. D 94, 042002 (2016).

[3] A. Singh, M. A. Papa, H.-B. Eggenstein, S. Zhu, H. Pletsch, B. Allen, O. Bock, B. Maschenchalk, R. Prix, and X. Siemens, Results of an all-sky high-frequency Einstein@ Home search for continuous gravitational waves in LIGO fifth science run, Phys. Rev. D 94, 064061 (2016).

[4] J. Aasi et al. (LIGO Scientific and Virgo Collaborations), First low frequency all-sky search for continuous gravitational wave signals, Phys. Rev. D 93, 042007 (2016).

[5] B.P. Abbott et al. (LIGO Scientific Collaboration), Einstein@Home all-sky search for periodic gravitational waves in LIGO S5 data, Phys. Rev. D 87, 042001 (2013).

[6] J. Abadie et al. (LIGO Scientific and Virgo Collaborations), All-sky search for periodic gravitational waves in the full S5 data, Phys. Rev. D 85, 022001 (2012).

[7] B. P. Abbott et al. (LIGO Scientific Collaboration), Einstein@Home search for periodic gravitational waves in early S5 LIGO data, Phys. Rev. D 80, 042003 (2009).

[8] B. P. Abbott et al. (LIGO Scientific Collaboration), All-sky LIGO search for periodic gravitational waves in the early S5 data, Phys. Rev. Lett. 102, 111102 (2009).

[9] B. Abbott et al. (LIGO Scientific Collaboration), All-sky search for periodic gravitational waves in LIGO S4 data, Phys. Rev. D 77, 022001 (2008).

[10] B. Abbott et al. (LIGO Scientific Collaboration), Einstein@Home search for periodic gravitational waves in LIGO S4 data, Phys. Rev. D 79, 022001 (2009).

[11] B. Abbott et al. (LIGO Scientific Collaboration), Searches for periodic gravitational waves from unknown isolated sources and Scorpius X-1: Results from the second LIGO science run, Phys. Rev. D 76, 082001 (2007).

[12] B. P. Abbott et al. (LIGO Scientific Collaboration), LIGO: The laser interferometer gravitational-wave observatory, Rep. Prog. Phys. 72, 076901 (2009).
[13] J. Abadie et al. (LIGO Scientific and Virgo Collaborations), Sensitivity achieved by the LIGO and Virgo gravitational wave detectors during LIGO's sixth and Virgo's second and third science runs, arXiv:1203.2674.

[14] J. Aasi et al. (LIGO Scientific Collaboration), Advanced LIGO, Classical Quantum Gravity 32, 074001 (2015).

[15] M. Shaltev, Optimizing the StackSlide setup and data selection for continuous-gravitational-wave searches in realistic detector data, Phys. Rev. D 93, 044058, 2016.

[16] J. Aasi et al. (LIGO Scientific and Virgo Collaborations), Characterization of the LIGO detectors during their sixth science run, Classical Quantum Gravity 32, 115012 (2015).

[17] H. J. Pletsch, Parameter-space correlations of the optimal statistic for continuous gravitational-wave detection, Phys. Rev. D 78, 102005 (2008).

[18] H. J. Pletsch, Parameter-space metric of semicoherent searches for continuous gravitational waves, Phys. Rev. D 82, 042002 (2010).

[19] C. Cutler and B.F. Schutz, The generalized $\mathcal{F}$-statistic: multiple detectors and multiple gravitational wave pulsars, Phys. Rev. D 72, 063006 (2005).

[20] J. Aasi et al. (LIGO Scientific and Virgo Collaborations), Directed search for continuous gravitational waves from the Galactic Center, Phys. Rev. D 88, 102002 (2013).

[21] D. Keitel, R. Prix, M. A. Papa, P. Leaci, and M. Siddiqi, Search for continuous gravitational waves: Improving robustness versus instrumental artifacts, Phys. Rev. D 89, 064023 (2014).

[22] B. Behnke, M. A. Papa, and R. Prix, Postprocessing methods used in the search for continuous gravitationalwave signals from the Galactic Center, Phys. Rev. D 91, 064007 (2015).

[23] Karl Wette, Estimating the sensitivity of wide-parameterspace searches for gravitational-wave pulsars, Phys. Rev. D 85, 042003 (2012).

[24] J. Ming, B. Krishnan, M. A. Papa, C. Aulbert, and H. Fehrmann, Optimal directed searches for continuous gravitational waves, Phys. Rev. D 93, 064011 (2016). 
B. P. Abbott, ${ }^{1}$ R. Abbott, ${ }^{1}$ T. D. Abbott, ${ }^{2}$ M. R. Abernathy, ${ }^{3}$ F. Acernese,${ }^{4,5}$ K. Ackley, ${ }^{6}$ C. Adams, ${ }^{7}$ T. Adams,${ }^{8}$ P. Addesso, ${ }^{9}$ R. X. Adhikari, ${ }^{1}$ V. B. Adya,${ }^{10}$ C. Affeldt, ${ }^{10}$ M. Agathos, ${ }^{11}$ K. Agatsuma,${ }^{11}$ N. Aggarwal, ${ }^{12}$ O. D. Aguiar,${ }^{13}$ L. Aiello,,${ }^{14,15}$ A. Ain, ${ }^{16}$ B. Allen, ${ }^{10,18,19}$ A. Allocca,${ }^{20,21}$ P. A. Altin, ${ }^{22}$ S. B. Anderson, ${ }^{1}$ W. G. Anderson, ${ }^{18}$ K. Arai, ${ }^{1}$ M. C. Araya, ${ }^{1}$ C. C. Arceneaux, ${ }^{23}$ J. S. Areeda, ${ }^{24}$ N. Arnaud ${ }^{25}$ K. G. Arun, ${ }^{26}$ S. Ascenzi, ${ }^{27,15}$ G. Ashton, ${ }^{28}$ M. Ast, ${ }^{29}$ S. M. Aston, ${ }^{7}$ P. Astone,${ }^{30}$ P. Aufmuth, ${ }^{19}$ C. Aulbert, ${ }^{10}$ S. Babak, ${ }^{31}$ P. Bacon, ${ }^{32}$ M. K. M. Bader, ${ }^{11}$ P. T. Baker, ${ }^{33}$ F. Baldaccini,${ }^{34,35}$ G. Ballardin, ${ }^{36}$ S. W. Ballmer, ${ }^{37}$ J. C. Barayoga, ${ }^{1}$ S. E. Barclay, ${ }^{38}$ B. C. Barish, ${ }^{1}$ D. Barker,${ }^{39}$ F. Barone, ${ }^{45}$ B. Barr, ${ }^{38}$ L. Barsotti, ${ }^{12}$ M. Barsuglia, ${ }^{32}$ D. Barta, ${ }^{40}$ J. Bartlett, ${ }^{39}$ I. Bartos, ${ }^{41}$ R. Bassiri, ${ }^{42}$ A. Basti, ${ }^{20,21}$ J. C. Batch,${ }^{39}$ C. Baune,${ }^{10}$ V. Bavigadda, ${ }^{36}$ M. Bazzan, ${ }^{43,44}$ M. Bejger, ${ }^{45}$ A. S. Bell, ${ }^{38}$ B. K. Berger, ${ }^{1}$ G. Bergmann, ${ }^{10}$ C. P. L. Berry, ${ }^{46}$ D. Bersanetti, ${ }^{47,48}$ A. Bertolini, ${ }^{11}$ J. Betzwieser, ${ }^{7}$ S. Bhagwat, ${ }^{37}$ R. Bhandare, ${ }^{49}$ I. A. Bilenko, ${ }^{50}$ G. Billingsley, ${ }^{1}$ J. Birch, ${ }^{7}$ R. Birney,${ }^{51}$ S. Biscans, ${ }^{12}$ A. Bisht, ${ }^{10,19}$ M. Bitossi, ${ }^{36}$ C. Biwer, ${ }^{37}$ M. A. Bizouard, ${ }^{25}$ J. K. Blackburn, ${ }^{1}$ C. D. Blair, ${ }^{52}$ D. G. Blair, ${ }^{52}$ R. M. Blair, ${ }^{39}$ S. Bloemen, ${ }^{53}$ O. Bock,${ }^{10}$ M. Boer, ${ }^{54}$ G. Bogaert, ${ }^{54}$ C. Bogan,${ }^{10}$ A. Bohe,${ }^{31}$ C. Bond,${ }^{46}$ F. Bondu, ${ }^{55}$ R. Bonnand, ${ }^{8}$ B. A. Boom,${ }^{11}$ R. Bork, ${ }^{1}$ V. Boschi, ${ }^{20,21}$ S. Bose,${ }^{56,16}$ Y. Bouffanais,${ }^{32}$ A. Bozzi,${ }^{36}$ C. Bradaschia,${ }^{21}$ P. R. Brady, ${ }^{18}$ V. B. Braginsky, ${ }^{50 \dagger}$ M. Branchesi,${ }^{57,58}$ J. E. Brau, ${ }^{59}$ T. Briant, ${ }^{60}$ A. Brillet,${ }^{54}$ M. Brinkmann,${ }^{10}$ V. Brisson, ${ }^{25}$ P. Brockill, ${ }^{18}$ J. E. Broida, ${ }^{61}$ A. F. Brooks, ${ }^{1}$ D. A. Brown, ${ }^{37}$ D. D. Brown, ${ }^{46}$ N. M. Brown, ${ }^{12}$ S. Brunett, ${ }^{1}$ C. C. Buchanan, ${ }^{2}$ A. Buikema, ${ }^{12}$ T. Bulik,${ }^{62}$ H. J. Bulten, ${ }^{63,11}$ A. Buonanno, ${ }^{31,64}$ D. Buskulic, ${ }^{8}$ C. Buy, ${ }^{32}$ R. L. Byer, ${ }^{42}$ M. Cabero, ${ }^{10}$ L. Cadonati, ${ }^{65}$ G. Cagnoli ${ }^{66,67}$ C. Cahillane, ${ }^{1}$ J. Calderón Bustillo, ${ }^{65}$ T. Callister, ${ }^{1}$ E. Calloni, ${ }^{68,5}$ J. B. Camp, ${ }^{69}$ K. C. Cannon, ${ }^{70}$ J. Cao, ${ }^{71}$ C. D. Capano, ${ }^{10}$ E. Capocasa, ${ }^{32}$ F. Carbognani, ${ }^{36}$ S. Caride, ${ }^{72}$ J. Casanueva Diaz ${ }^{25}$ C. Casentini, ${ }^{27,15}$ S. Caudill, ${ }^{18}$ M. Cavaglià, ${ }^{23}$ F. Cavalier ${ }^{25}$ R. Cavalieri, ${ }^{36}$ G. Cella, ${ }^{21}$ C. B. Cepeda, ${ }^{1}$ L. Cerboni Baiardi, ${ }^{57,58}$ G. Cerretani, ${ }^{20,21}$ E. Cesarini, ${ }^{27,15}$ S. J. Chamberlin, ${ }^{73}$ M. Chan, ${ }^{38}$ S. Chao, ${ }^{74}$ P. Charlton, ${ }^{75}$ E. Chassande-Mottin, ${ }^{32}$ B. D. Cheeseboro, ${ }^{76}$ H. Y. Chen, ${ }^{77}$ Y. Chen,${ }^{78}$ C. Cheng, ${ }^{74}$ A. Chincarini, ${ }^{48}$ A. Chiummo, ${ }^{36}$ H. S. Cho, ${ }^{79}$ M. Cho, ${ }^{64}$ J. H. Chow, ${ }^{22}$ N. Christensen, ${ }^{61}$ Q. Chu, ${ }^{52}$ S. Chua, ${ }^{60}$ S. Chung, ${ }^{52}$ G. Ciani, ${ }^{6}$ F. Clara, ${ }^{39}$ J. A. Clark, ${ }^{65}$ F. Cleva ${ }^{54}$ E. Coccia, ${ }^{27,14}$ P.-F. Cohadon,${ }^{60}$ A. Colla,${ }^{80,30}$ C. G. Collette, ${ }^{81}$ L. Cominsky, ${ }^{82}$ M. Constancio Jr., ${ }^{13}$ A. Conte, ${ }^{80,30}$ L. Conti, ${ }^{44}$ D. Cook ${ }^{39}$ T. R. Corbitt, ${ }^{2}$ N. Cornish, ${ }^{33}$ A. Corsi,${ }^{72}$ S. Cortese, ${ }^{36}$ C. A. Costa, ${ }^{13}$ M. W. Coughlin, ${ }^{61}$ S. B. Coughlin, ${ }^{83}$ J.-P. Coulon, ${ }^{54}$ S. T. Countryman, ${ }^{41}$ P. Couvares, ${ }^{1}$ E. E. Cowan, ${ }^{65}$ D. M. Coward, ${ }^{52}$ M. J. Cowart, ${ }^{7}$ D. C. Coyne, ${ }^{1}$ R. Coyne,${ }^{72}$ K. Craig, ${ }^{38}$ J. D. E. Creighton, ${ }^{18}$ T. D. Creighton, ${ }^{88}$ J. Cripe, ${ }^{2}$ S. G. Crowder,${ }^{84}$ A. Cumming ${ }^{38}$ L. Cunningham, ${ }^{38}$ E. Cuoco, ${ }^{36}$ T. Dal Canton, ${ }^{10}$ S. L. Danilishin, ${ }^{38}$ S. D’ Antonio, ${ }^{15}$ K. Danzmann, ${ }^{19,10}$ N. S. Darman, ${ }^{85}$ A. Dasgupta, ${ }^{86}$ C. F. Da Silva Costa, ${ }^{6}$ V. Dattilo, ${ }^{36}$ I. Dave, ${ }^{49}$ M. Davier, ${ }^{25}$ G. S. Davies, ${ }^{38}$ E. J. Daw, ${ }^{87}$ R. Day, ${ }^{36}$ S. De,${ }^{37}$ D. DeBra, ${ }^{42}$ G. Debreczeni, ${ }^{40}$ J. Degallaix,${ }^{66}$ M. De Laurentis, ${ }^{68,5}$ S. Deléglise, ${ }^{60}$ W. Del Pozzo,${ }^{46}$ T. Denker,${ }^{10}$ T. Dent,${ }^{10}$ V. Dergachev, ${ }^{1}$ R. De Rosa, ${ }^{68,5}$ R. T. DeRosa, ${ }^{7}$ R. DeSalvo, ${ }^{9}$ R. C. Devine,${ }^{76}$ S. Dhurandhar,${ }^{16}$ M. C. Díaz ${ }^{88}$ L. Di Fiore,${ }^{5}$ M. Di Giovanni, ${ }^{89,90}$ T. Di Girolamo, ${ }^{68,5}$ A. Di Lieto, ${ }^{20,21}$ S. Di Pace, ${ }^{80,30}$ I. Di Palma, ${ }^{31,80,30}$ A. Di Virgilio, ${ }^{21}$ V. Dolique, ${ }^{66}$ F. Donovan, ${ }^{12}$ K. L. Dooley, ${ }^{23}$ S. Doravari, ${ }^{10}$ R. Douglas, ${ }^{38}$ T. P. Downes, ${ }^{18}$ M. Drago, ${ }^{10}$ R. W. P. Drever, ${ }^{1}$ J. C. Driggers,${ }^{39}$ M. Ducrot, ${ }^{8}$ S. E. Dwyer, ${ }^{39}$ T. B. Edo, ${ }^{87}$ M. C. Edwards, ${ }^{61}$ A. Effler, ${ }^{7}$ H.-B. Eggenstein, ${ }^{10}$ P. Ehrens, ${ }^{1}$ J. Eichholz, ${ }^{6,1}$ S. S. Eikenberry, ${ }^{6}$ W. Engels, ${ }^{78}$ R. C. Essick, ${ }^{12}$ T. Etzel, ${ }^{1}$ M. Evans, ${ }^{12}$ T. M. Evans, ${ }^{7}$ R. Everett, ${ }^{73}$ M. Factourovich, ${ }^{41}$ V. Fafone,${ }^{27,15}$ H. Fair, ${ }^{37}$ S. Fairhurst, ${ }^{91}$ X. Fan, ${ }^{71}$ Q. Fang, ${ }^{52}$ S. Farinon,${ }^{48}$ B. Farr, ${ }^{77}$ W. M. Farr, ${ }^{46}$ M. Favata, ${ }^{92}$ M. Fays,${ }^{91}$ H. Fehrmann, ${ }^{10}$ M. M. Fejer, ${ }^{42}$ E. Fenyvesi, ${ }^{93}$ I. Ferrante, ${ }^{20,21}$ E. C. Ferreira, ${ }^{13}$ F. Ferrini, ${ }^{36}$ F. Fidecaro, ${ }^{20,21}$ I. Fiori, ${ }^{36}$ D. Fiorucci, ${ }^{32}$ R. P. Fisher, ${ }^{37}$ R. Flaminio, ${ }^{66,94}$ M. Fletcher, ${ }^{38}$ J.-D. Fournier, ${ }^{54}$ S. Frasca, ${ }^{80,30}$ F. Frasconi, ${ }^{21}$ Z. Frei, ${ }^{93}$ A. Freise, ${ }^{46}$ R. Frey, ${ }^{59}$ V. Frey, ${ }^{25}$ P. Fritschel, ${ }^{12}$ V. V. Frolov, ${ }^{7}$ P. Fulda, ${ }^{6}$ M. Fyffe, ${ }^{7}$ H. A. G. Gabbard ${ }^{23}$ J. R. Gair, ${ }^{95}$ L. Gammaitoni, ${ }^{34}$ S. G. Gaonkar, ${ }^{16}$ F. Garufi, ${ }^{68,5}$ G. Gaur, ${ }^{96,86}$ N. Gehrels, ${ }^{69}$ G. Gemme, ${ }^{48}$ P. Geng, ${ }^{88}$ E. Genin, ${ }^{36}$ A. Gennai, ${ }^{21}$ J. George, ${ }^{49}$ L. Gergely, ${ }^{97}$ V. Germain, ${ }^{8}$ Abhirup Ghosh,${ }^{17}$ Archisman Ghosh, ${ }^{17}$ S. Ghosh, ${ }^{53,11}$ J. A. Giaime, ${ }^{2,7}$ K. D. Giardina, ${ }^{7}$ A. Giazotto, ${ }^{21}$ K. Gill, ${ }^{98}$ A. Glaefke, ${ }^{38}$ E. Goetz, ${ }^{39}$ R. Goetz, ${ }^{6}$ L. Gondan, ${ }^{93}$ G. González, ${ }^{2}$ J. M. Gonzalez Castro, ${ }^{20,21}$ A. Gopakumar, ${ }^{99}$ N. A. Gordon, ${ }^{38}$ M. L. Gorodetsky, ${ }^{50}$ S. E. Gossan, ${ }^{1}$ M. Gosselin, ${ }^{36}$ R. Gouaty, ${ }^{8}$ A. Grado, ${ }^{100,5}$ C. Graef, ${ }^{38}$ P. B. Graff, ${ }^{64}$ M. Granata, ${ }^{66}$ A. Grant, ${ }^{38}$ S. Gras, ${ }^{12}$ C. Gray, ${ }^{39}$ G. Greco, ${ }^{57,58}$ A. C. Green, ${ }^{46}$ P. Groot, ${ }^{53}$ H. Grote, ${ }^{10}$ S. Grunewald ${ }^{31}$ G. M. Guidi, ${ }^{57,58}$ X. Guo, ${ }^{71}$ A. Gupta, ${ }^{16}$ M. K. Gupta, ${ }^{86}$ K. E. Gushwa, ${ }^{1}$ E. K. Gustafson, ${ }^{1}$ R. Gustafson, ${ }^{101}$ J. J. Hacker,${ }^{24}$ B. R. Hall, ${ }^{56}$ E. D. Hall, ${ }^{1}$ G. Hammond, ${ }^{38}$ M. Haney, ${ }^{99}$ M. M. Hanke, ${ }^{10}$ J. Hanks, ${ }^{39}$ C. Hanna, ${ }^{73}$ J. Hanson, ${ }^{7}$ T. Hardwick, ${ }^{2}$ J. Harms,${ }^{57,58}$ G. M. Harry ${ }^{3}$ I. W. Harry,${ }^{31}$ M. J. Hart, ${ }^{38}$ M. T. Hartman, ${ }^{6}$ C.-J. Haster ${ }^{46}$ K. Haughian, ${ }^{38}$ A. Heidmann, ${ }^{60}$ M. C. Heintze, ${ }^{7}$ H. Heitmann, ${ }^{54}$ P. Hello, ${ }^{25}$ G. Hemming,${ }^{36}$ M. Hendry, ${ }^{38}$ I. S. Heng, ${ }^{38}$ J. Hennig, ${ }^{38}$ J. Henry, ${ }^{102}$ A. W. Heptonstall, ${ }^{1}$ M. Heurs, ${ }^{10,19}$ S. Hild ${ }^{38}$ D. Hoak,${ }^{36}$ D. Hofman,${ }^{66}$ K. Holt, ${ }^{7}$ D. E. Holz,${ }^{77}$ P. Hopkins, ${ }^{91}$ J. Hough,${ }^{38}$ E. A. Houston, ${ }^{38}$ E. J. Howell, ${ }^{52}$ Y. M. Hu, ${ }^{10}$ S. Huang, ${ }^{74}$ E. A. Huerta, ${ }^{103}$ D. Huet,${ }^{25}$ B. Hughey, ${ }^{98}$ S. Husa, ${ }^{104}$ S. H. Huttner, ${ }^{38}$ T. Huynh-Dinh, ${ }^{7}$ N. Indik, ${ }^{10}$ D. R. Ingram, ${ }^{39}$ R. Inta, ${ }^{72}$ H. N. Isa, ${ }^{38}$ J.-M. Isac, ${ }^{60}$ 
M. Isi, ${ }^{1}$ T. Isogai, ${ }^{12}$ B. R. Iyer, ${ }^{17}$ K. Izumi, ${ }^{39}$ T. Jacqmin, ${ }^{60}$ H. Jang, ${ }^{79}$ K. Jani, ${ }^{65}$ P. Jaranowski, ${ }^{105}$ S. Jawahar, ${ }^{106}$ L. Jian, ${ }^{52}$ F. Jiménez-Forteza, ${ }^{104}$ W. W. Johnson, ${ }^{2}$ D. I. Jones,${ }^{28}$ R. Jones,${ }^{38}$ R. J. G. Jonker, ${ }^{11}$ L. Ju, ${ }^{52}$ Haris K, ${ }^{107}$ C. V. Kalaghatgi, ${ }^{91}$

V. Kalogera, ${ }^{83}$ S. Kandhasamy, ${ }^{23}$ G. Kang, ${ }^{79}$ J. B. Kanner, ${ }^{1}$ S. J. Kapadia, ${ }^{10}$ S. Karki, ${ }^{59}$ K. S. Karvinen, ${ }^{10}$ M. Kasprzack, ${ }^{36,2}$ E. Katsavounidis, ${ }^{12}$ W. Katzman, ${ }^{7}$ S. Kaufer, ${ }^{19}$ T. Kaur, ${ }^{52}$ K. Kawabe, ${ }^{39}$ F. Kéfélian, ${ }^{54}$ M. S. Kehl, ${ }^{108}$ D. Keitel, ${ }^{104}$

D. B. Kelley, ${ }^{37}$ W. Kells, ${ }^{1}$ R. Kennedy,${ }^{87}$ J. S. Key, ${ }^{88}$ F. Y. Khalili,${ }^{50}$ I. Khan,${ }^{14}$ S. Khan, ${ }^{91}$ Z. Khan, ${ }^{86}$ E. A. Khazanov, ${ }^{109}$ N. Kijbunchoo, ${ }^{39}$ Chi-Woong Kim, ${ }^{79}$ Chunglee Kim, ${ }^{79}$ J. Kim, ${ }^{110}$ K. Kim, ${ }^{111}$ N. Kim, ${ }^{42}$ W. Kim, ${ }^{112}$ Y.-M. Kim, ${ }^{110}$ S. J. Kimbrell, ${ }^{65}$ E. J. King, ${ }^{112}$ P. J. King, ${ }^{39}$ J. S. Kissel, ${ }^{39}$ B. Klein, ${ }^{83}$ L. Kleybolte, ${ }^{29}$ S. Klimenko, ${ }^{6}$ S. M. Koehlenbeck, ${ }^{10}$ S. Koley, ${ }^{11}$ V. Kondrashov, ${ }^{1}$ A. Kontos, ${ }^{12}$ M. Korobko, ${ }^{29}$ W. Z. Korth, ${ }^{1}$ I. Kowalska, ${ }^{62}$ D. B. Kozak, ${ }^{1}$ V. Kringel, ${ }^{10}$ B. Krishnan, ${ }^{10}$ A. Królak, ${ }^{113,114}$ C. Krueger, ${ }^{19}$ G. Kuehn, ${ }^{10}$ P. Kumar, ${ }^{108}$ R. Kumar, ${ }^{86}$ L. Kuo, ${ }^{74}$ A. Kutynia, ${ }^{113}$ B. D. Lackey, ${ }^{37}$ M. Landry, ${ }^{39}$ J. Lange, ${ }^{102}$ B. Lantz, ${ }^{42}$ P. D. Lasky, ${ }^{115}$ M. Laxen, ${ }^{7}$ C. Lazzaro, ${ }^{44}$ P. Leaci, ${ }^{80,30}$ S. Leavey, ${ }^{38}$ E. O. Lebigot, ${ }^{32,71}$ C. H. Lee, ${ }^{110}$ H. K. Lee, ${ }^{111}$ H. M. Lee, ${ }^{116}$ K. Lee, ${ }^{38}$ A. Lenon, ${ }^{37}$ M. Leonardi, ${ }^{89,90}$ J. R. Leong, ${ }^{10}$ N. Leroy ${ }^{25}$ N. Letendre, ${ }^{8}$ Y. Levin, ${ }^{115}$ J. B. Lewis, ${ }^{1}$ T. G. F. Li, ${ }^{117}$ A. Libson, ${ }^{12}$ T. B. Littenberg, ${ }^{118}$ N. A. Lockerbie, ${ }^{106}$ A. L. Lombardi, ${ }^{119}$ L. T. London, ${ }^{91}$ J. E. Lord, ${ }^{37}$ M. Lorenzini, ${ }^{14,15}$ V. Loriette, ${ }^{120}$ M. Lormand,${ }^{7}$ G. Losurdo, ${ }^{58}$ J. D. Lough, ${ }^{10,19}$ H. Lück, ${ }^{19,10}$ A. P. Lundgren, ${ }^{10}$ R. Lynch, ${ }^{12}$ Y. Ma,${ }^{52}$ B. Machenschalk, ${ }^{10}$ M. MacInnis, ${ }^{12}$ D. M. Macleod, ${ }^{2}$ F. Magaña-Sandoval,${ }^{37}$ L. Magaña Zertuche,${ }^{37}$ R. M. Magee,${ }^{56}$ E. Majorana,${ }^{30}$ I. Maksimovic, ${ }^{120}$ V. Malvezzi, ${ }^{27,15}$ N. Man,${ }^{54}$ V. Mandic,${ }^{84}$ V. Mangano, ${ }^{38}$ G. L. Mansell, ${ }^{22}$ M. Manske,${ }^{18}$ M. Mantovani,${ }^{36}$ F. Marchesoni, ${ }^{121,35}$ F. Marion, ${ }^{8}$ S. Márka, ${ }^{41}$ Z. Márka, ${ }^{41}$ A. S. Markosyan, ${ }^{42}$ E. Maros, ${ }^{1}$ F. Martelli, ${ }^{57,58}$ L. Martellini,${ }^{54}$ I. W. Martin ${ }^{38}$ D. V. Martynov, ${ }^{12}$ J. N. Marx, ${ }^{1}$

K. Mason, ${ }^{12}$ A. Masserot ${ }^{8}$ T. J. Massinger, ${ }^{37}$ M. Masso-Reid, ${ }^{38}$ S. Mastrogiovanni, ${ }^{80,30}$ F. Matichard, ${ }^{12}$ L. Matone, ${ }^{41}$ N. Mavalvala, ${ }^{12}$ N. Mazumder, ${ }^{56}$ R. McCarthy, ${ }^{39}$ D. E. McClelland, ${ }^{22}$ S. McCormick, ${ }^{7}$ S. C. McGuire, ${ }^{122}$ G. McIntyre, ${ }^{1}$ J. McIver, ${ }^{1}$ D. J. McManus, ${ }^{22}$ T. McRae, ${ }^{22}$ S. T. McWilliams, ${ }^{76}$ D. Meacher, ${ }^{73}$ G. D. Meadors ${ }^{31,10}$ J. Meidam, ${ }^{11}$ A. Melatos,${ }^{85}$ G. Mendell, ${ }^{39}$ R. A. Mercer, ${ }^{18}$ E. L. Merilh, ${ }^{39}$ M. Merzougui, ${ }^{54}$ S. Meshkov, ${ }^{1}$ C. Messenger,${ }^{38}$ C. Messick,${ }^{73}$ R. Metzdorff, ${ }^{60}$ P. M. Meyers,${ }^{84}$ F. Mezzani,${ }^{30,80}$ H. Miao,${ }^{46}$ C. Michel,${ }^{66}$ H. Middleton, ${ }^{46}$ E. E. Mikhailov, ${ }^{123}$ L. Milano, ${ }^{68,5}$ A. L. Miller, ${ }^{6,80,30}$ A. Miller, ${ }^{83}$ B. B. Miller, ${ }^{83}$ J. Miller, ${ }^{12}$ M. Millhouse, ${ }^{33}$ Y. Minenkov, ${ }^{15}$ J. Ming, ${ }^{31}$ S. Mirshekari, ${ }^{124}$ C. Mishra, ${ }^{17}$ S. Mitra, ${ }^{16}$ V. P. Mitrofanov, ${ }^{50}$ G. Mitselmakher, ${ }^{6}$ R. Mittleman, ${ }^{12}$ A. Moggi, ${ }^{21}$ M. Mohan, ${ }^{36}$ S. R. P. Mohapatra, ${ }^{12}$ M. Montani, ${ }^{57,58}$ B. C. Moore, ${ }^{92}$ C. J. Moore, ${ }^{125}$ D. Moraru, ${ }^{39}$ G. Moreno, ${ }^{39}$ S. R. Morriss, ${ }^{88}$ K. Mossavi, ${ }^{10}$ B. Mours, ${ }^{8}$ C. M. Mow-Lowry, ${ }^{46}$ G. Mueller, ${ }^{6}$ A. W. Muir, ${ }^{11}$ Arunava Mukherjee, ${ }^{17}$ D. Mukherjee,${ }^{18}$ S. Mukherjee, ${ }^{88}$ N. Mukund, ${ }^{16}$ A. Mullavey, ${ }^{7}$ J. Munch, ${ }^{112}$ D. J. Murphy, ${ }^{41}$ P. G. Murray, ${ }^{38}$ A. Mytidis,${ }^{6}$ I. Nardecchia, ${ }^{27,15}$ L. Naticchioni, ${ }^{80,30}$ R. K. Nayak, ${ }^{126}$ K. Nedkova, ${ }^{119}$ G. Nelemans, ${ }^{53,11}$ T. J. N. Nelson, ${ }^{7}$ M. Neri, ${ }^{47,48}$ A. Neunzert, ${ }^{101}$ G. Newton, ${ }^{38}$ T. T. Nguyen, ${ }^{22}$ A. B. Nielsen, ${ }^{10}$ S. Nissanke, ${ }^{53,11}$ A. Nitz ${ }^{10}$ F. Nocera, ${ }^{36}$ D. Nolting, ${ }^{7}$ M. E. N. Normandin, ${ }^{88}$ L. K. Nuttall, ${ }^{37}$ J. Oberling, ${ }^{39}$ E. Ochsner, ${ }^{18}$ J. O’Dell, ${ }^{127}$ E. Oelker, ${ }^{12}$ G. H. Ogin, ${ }^{128}$ J. J. Oh, ${ }^{129}$ S. H. Oh, ${ }^{129}$ F. Ohme, ${ }^{91}$ M. Oliver, ${ }^{104}$ P. Oppermann, ${ }^{10}$ Richard J. Oram, ${ }^{7}$ B. O'Reilly, ${ }^{7}$ R. O'Shaughnessy, ${ }^{102}$ D. J. Ottaway, ${ }^{112}$ H. Overmier, ${ }^{7}$ B. J. Owen, ${ }^{72}$ A. Pai, ${ }^{107}$ S. A. Pai, ${ }^{49}$ J. R. Palamos, ${ }^{59}$ O. Palashov, ${ }^{109}$ C. Palomba ${ }^{30}$ A. Pal-Singh,${ }^{29}$ H. Pan, ${ }^{74}$ C. Pankow,${ }^{83}$ F. Pannarale, ${ }^{91}$ B. C. Pant, ${ }^{49}$ F. Paoletti, ${ }^{36,21}$ A. Paoli, ${ }^{36}$ M. A. Papa,${ }^{31,18,10}$ H. R. Paris, ${ }^{42}$ W. Parker, ${ }^{7}$ D. Pascucci, ${ }^{38}$ A. Pasqualetti ${ }^{36}$ R. Passaquieti, ${ }^{20,21}$ D. Passuello, ${ }^{21}$ B. Patricelli, ${ }^{20,21}$ Z. Patrick, ${ }^{42}$ B. L. Pearlstone,${ }^{38}$ M. Pedraza, ${ }^{1}$ R. Pedurand, ${ }^{66,130}$ L. Pekowsky, ${ }^{37}$ A. Pele, ${ }^{7}$ S. Penn, ${ }^{131}$ A. Perreca, ${ }^{1}$ L. M. Perri, ${ }^{83}$ M. Phelps,${ }^{38}$ O. J. Piccinni, ${ }^{80,30}$ M. Pichot, ${ }^{54}$ F. Piergiovanni, ${ }^{57,58}$ V. Pierro, ${ }^{9}$ G. Pillant, ${ }^{36}$ L. Pinard, ${ }^{66}$ I. M. Pinto, ${ }^{9}$ M. Pitkin,${ }^{38}$ M. Poe, ${ }^{18}$ R. Poggiani, ${ }^{20,21}$ P. Popolizio, ${ }^{36}$ A. Post,${ }^{10}$ J. Powell, ${ }^{38}$ J. Prasad,${ }^{16}$ V. Predoi,${ }^{91}$ T. Prestegard,${ }^{84}$ L. R. Price, ${ }^{1}$ M. Prijatelj,${ }^{10,36}$ M. Principe,${ }^{9}$ S. Privitera, ${ }^{31}$ R. Prix,${ }^{10}$ G. A. Prodi,${ }^{89,90}$ L. Prokhorov, ${ }^{50}$ O. Puncken,${ }^{10}$ M. Punturo, ${ }^{35}$ P. Puppo, ${ }^{30}$ M. Pürrer, ${ }^{31}$ H. Qi,${ }^{18}$ J. Qin, ${ }^{52}$ S. Qiu, ${ }^{115}$ V. Quetschke, ${ }^{88}$ E. A. Quintero, ${ }^{1}$ R. Quitzow-James, ${ }^{59}$ F. J. Raab, ${ }^{39}$ D. S. Rabeling, ${ }^{22}$ H. Radkins, ${ }^{39}$ P. Raffai, ${ }^{93}$ S. Raja, ${ }^{49}$ C. Rajan, ${ }^{49}$ M. Rakhmanov, ${ }^{88}$ P. Rapagnani, ${ }^{80,30}$ V. Raymond, ${ }^{31}$ M. Razzano, ${ }^{20,21}$ V. Re, ${ }^{27}$ J. Read, ${ }^{24}$ C. M. Reed ${ }^{39}$ T. Regimbau, ${ }^{54}$ L. Rei,${ }^{48}$ S. Reid,${ }^{51}$ D. H. Reitze, ${ }^{1,6}$ H. Rew, ${ }^{123}$ S. D. Reyes,${ }^{37}$ F. Ricci, ${ }^{80,30}$ K. Riles, ${ }^{101}$ M. Rizzo, ${ }^{102}$ N. A. Robertson, ${ }^{1,38}$ R. Robie, ${ }^{38}$ F. Robinet, ${ }^{25}$ A. Rocchi, ${ }^{15}$ L. Rolland ${ }^{8}$ J. G. Rollins, ${ }^{1}$ V. J. Roma, ${ }^{59}$ R. Romano, ${ }^{4,5}$ G. Romanov, ${ }^{123}$ J. H. Romie, ${ }^{7}$ D. Rosińska, ${ }^{132,45}$ S. Rowan, ${ }^{38}$ A. Rüdiger, ${ }^{10}$ P. Ruggi, ${ }^{36}$ K. Ryan, ${ }^{39}$ S. Sachdev, ${ }^{1}$ T. Sadecki, ${ }^{39}$ L. Sadeghian, ${ }^{18}$ M. Sakellariadou, ${ }^{133}$ L. Salconi,${ }^{36}$ M. Saleem, ${ }^{107}$ F. Salemi ${ }^{10}$ A. Samajdar, ${ }^{126}$ L. Sammut, ${ }^{115}$ E. J. Sanchez, ${ }^{1}$ V. Sandberg, ${ }^{39}$ B. Sandeen, ${ }^{83}$ J. R. Sanders, ${ }^{37}$ B. Sassolas, ${ }^{66}$ P. R. Saulson, ${ }^{37}$ O. E. S. Sauter, ${ }^{101}$ R. L. Savage ${ }^{39}$ A. Sawadsky, ${ }^{19}$ P. Schale,${ }^{59}$ R. Schilling, ${ }^{10, \dagger}$ J. Schmidt, ${ }^{10}$ P. Schmidt, ${ }^{178}$ R. Schnabel, ${ }^{29}$

R. M. S. Schofield, ${ }^{59}$ A. Schönbeck,$^{29}$ E. Schreiber, ${ }^{10}$ D. Schuette, ${ }^{10,19}$ B. F. Schutz, ${ }^{91,31}$ J. Scott, ${ }^{38}$ S. M. Scott, ${ }^{22}$ D. Sellers, ${ }^{7}$ A. S. Sengupta ${ }^{96}$ D. Sentenac, ${ }^{36}$ V. Sequino, ${ }^{27,15}$ A. Sergeev, ${ }^{109}$ Y. Setyawati, ${ }^{53,11}$ D. A. Shaddock, ${ }^{22}$ T. Shaffer, ${ }^{39}$ M. S. Shahriar, ${ }^{83}$ M. Shaltev, ${ }^{10}$ B. Shapiro, ${ }^{42}$ P. Shawhan, ${ }^{64}$ A. Sheperd, ${ }^{18}$ D. H. Shoemaker, ${ }^{12}$ D. M. Shoemaker, ${ }^{65}$ 
K. Siellez, ${ }^{65}$ X. Siemens, ${ }^{18}$ M. Sieniawska, ${ }^{45}$ D. Sigg, ${ }^{39}$ A. D. Silva, ${ }^{13}$ A. Singer, ${ }^{1}$ L. P. Singer, ${ }^{69}$ A. Singh,${ }^{31,10,19}$ R. Singh, ${ }^{2}$ A. Singhal, ${ }^{14}$ A. M. Sintes, ${ }^{104}$ B. J. J. Slagmolen, ${ }^{22}$ J. R. Smith, ${ }^{24}$ N. D. Smith, ${ }^{1}$ R. J. E. Smith, ${ }^{1}$ E. J. Son, ${ }^{129}$ B. Sorazu ${ }^{38}$ F. Sorrentino, ${ }^{48}$ T. Souradeep, ${ }^{16}$ A. K. Srivastava, ${ }^{86}$ A. Staley, ${ }^{41}$ M. Steinke, ${ }^{10}$ J. Steinlechner, ${ }^{38}$ S. Steinlechner, ${ }^{38}$

D. Steinmeyer, ${ }^{10,19}$ B. C. Stephens, ${ }^{18}$ R. Stone,${ }^{88}$ K. A. Strain, ${ }^{38}$ N. Straniero, ${ }^{66}$ G. Stratta,${ }^{57,58}$ N. A. Strauss, ${ }^{61}$ S. Strigin, ${ }^{50}$ R. Sturani, ${ }^{124}$ A. L. Stuver, ${ }^{7}$ T. Z. Summerscales, ${ }^{134}$ L. Sun, ${ }^{85}$ S. Sunil, ${ }^{86}$ P. J. Sutton, ${ }^{91}$ B. L. Swinkels, ${ }^{36}$

M. J. Szczepańczyk ${ }^{98}$ M. Tacca, ${ }^{32}$ D. Talukder, ${ }^{59}$ D. B. Tanner, ${ }^{6}$ M. Tápai,${ }^{97}$ S. P. Tarabrin, ${ }^{10}$ A. Taracchini, ${ }^{31}$ R. Taylor, ${ }^{1}$

T. Theeg, ${ }^{10}$ M. P. Thirugnanasambandam, ${ }^{1}$ E. G. Thomas, ${ }^{46}$ M. Thomas, ${ }^{7}$ P. Thomas,${ }^{39}$ K. A. Thorne, ${ }^{7}$ E. Thrane,${ }^{115}$ S. Tiwari, ${ }^{14,90}$ V. Tiwari, ${ }^{91}$ K. V. Tokmakov,${ }^{106} \mathrm{~K}$. Toland,${ }^{38} \mathrm{C}$. Tomlinson, ${ }^{87} \mathrm{M}$. Tonelli, ${ }^{20,21} \mathrm{Z}$. Tornasi,${ }^{38} \mathrm{C}$. V. Torres,${ }^{88, \dagger}$ C. I. Torrie, ${ }^{1}$ D. Töyrä, ${ }^{46}$ F. Travasso, ${ }^{34,35}$ G. Traylor, ${ }^{7}$ D. Trifirò, ${ }^{23}$ M. C. Tringali, ${ }^{89,90}$ L. Trozzo, ${ }^{135,21}$ M. Tse, ${ }^{12}$ M. Turconi, ${ }^{54}$ D. Tuyenbayev, ${ }^{88}$ D. Ugolini, ${ }^{136}$ C. S. Unnikrishnan, ${ }^{99}$ A. L. Urban, ${ }^{18}$ S. A. Usman, ${ }^{37}$ H. Vahlbruch, ${ }^{19}$ G. Vajente, ${ }^{1}$ G. Valdes, ${ }^{88}$ N. van Bakel, ${ }^{11}$ M. van Beuzekom, ${ }^{11}$ J. F. J. van den Brand, ${ }^{63,11}$ C. Van Den Broeck, ${ }^{11}$ D. C. Vander-Hyde, ${ }^{37}$ L. van der Schaaf, ${ }^{11}$ J. V. van Heijningen, ${ }^{11}$ A. A. van Veggel, ${ }^{38}$ M. Vardaro, ${ }^{43,44}$ S. Vass, ${ }^{1}$ M. Vasúth, ${ }^{40}$ R. Vaulin, ${ }^{12}$ A. Vecchio,${ }^{46}$ G. Vedovato, ${ }^{44}$ J. Veitch,${ }^{46}$ P. J. Veitch,${ }^{12}$ K. Venkateswara, ${ }^{137}$ D. Verkindt, ${ }^{8}$ F. Vetrano ${ }^{57,58}$ A. Viceré ${ }^{57,58}$ S. Vinciguerra, ${ }^{46}$ D. J. Vine, ${ }^{51}$ J.-Y. Vinet, ${ }^{54}$ S. Vitale, ${ }^{12}$ T. Vo,${ }^{37}$ H. Vocca, ${ }^{34,35}$ C. Vorvick, ${ }^{39}$ D. V. Voss ${ }^{6}$ W. D. Vousden, ${ }^{46}$ S. P. Vyatchanin,${ }^{50}$ A. R. Wade, ${ }^{22}$ L. E. Wade,${ }^{138}$ M. Wade,${ }^{138}$ M. Walker, ${ }^{2}$ L. Wallace, ${ }^{1}$ S. Walsh,${ }^{31,10}$ G. Wang, ${ }^{14,58}$ H. Wang, ${ }^{46}$ M. Wang, ${ }^{46}$ X. Wang, ${ }^{71}$ Y. Wang, ${ }^{52}$ R. L. Ward, ${ }^{22}$ J. Warner, ${ }^{39}$ M. Was, ${ }^{8}$ B. Weaver,${ }^{39}$ L.-W. Wei, ${ }^{54}$ M. Weinert, ${ }^{10}$ A. J. Weinstein, ${ }^{1}$ R. Weiss, ${ }^{12}$ L. Wen, ${ }^{52}$ P. Weßels, ${ }^{10}$ T. Westphal, ${ }^{10}$ K. Wette, ${ }^{10}$ J. T. Whelan, ${ }^{102}$ B. F. Whiting, ${ }^{6}$ R. D. Williams, ${ }^{1}$ A. R. Williamson, ${ }^{91}$ J. L. Willis, ${ }^{139}$ B. Willke, ${ }^{19,10}$ M. H. Wimmer, ${ }^{10,19}$ W. Winkler, ${ }^{10}$ C. C. Wipf, ${ }^{1}$ H. Wittel, ${ }^{10,19}$ G. Woan, ${ }^{38}$ J. Woehler, ${ }^{10}$ J. Worden,,${ }^{39}$ J. L. Wright, ${ }^{38}$ D. S. Wu, ${ }^{10}$ G. Wu, ${ }^{7}$ J. Yablon, ${ }^{83}$ W. Yam, ${ }^{12}$ H. Yamamoto, ${ }^{1}$ C. C. Yancey, ${ }^{64}$ H. Yu ${ }^{12}$ M. Yvert, ${ }^{8}$ A. Zadrożny, ${ }^{113}$ L. Zangrando, ${ }^{44}$ M. Zanolin, ${ }^{98}$ J.-P. Zendri, ${ }^{44}$ M. Zevin, ${ }^{83}$ L. Zhang, ${ }^{1}$ M. Zhang, ${ }^{123}$ Y. Zhang, ${ }^{102}$ C. Zhao, ${ }^{52}$ M. Zhou, ${ }^{83}$ Z. Zhou, ${ }^{83}$ S. J. Zhu, ${ }^{31,10}$ X. Zhu, ${ }^{52}$ M. E. Zucker, ${ }^{1,12}$ S. E. Zuraw, ${ }^{119}$ and J. Zweizig ${ }^{1}$

(LIGO Scientific Collaboration and Virgo Collaboration)

${ }^{1}$ LIGO, California Institute of Technology, Pasadena, California 91125, USA

${ }^{2}$ Louisiana State University, Baton Rouge, Louisiana 70803, USA

${ }^{3}$ American University, Washington, D.C. 20016, USA

${ }^{4}$ Università di Salerno, Fisciano, I-84084 Salerno, Italy

${ }^{5}$ INFN, Sezione di Napoli, Complesso Universitario di Monte S. Angelo, I-80126 Napoli, Italy

${ }^{6}$ University of Florida, Gainesville, Florida 32611, USA

${ }^{7}$ LIGO Livingston Observatory, Livingston, Louisiana 70754, USA

${ }^{8}$ Laboratoire d'Annecy-le-Vieux de Physique des Particules (LAPP), Université Savoie Mont Blanc, CNRS/IN2P3, F-74941 Annecy-le-Vieux, France

${ }^{9}$ University of Sannio at Benevento, I-82100 Benevento, Italy and INFN, Sezione di Napoli, I-80100 Napoli, Italy

${ }^{10}$ Albert-Einstein-Institut, Max-Planck-Institut für Gravitationsphysik, D-30167 Hannover, Germany

${ }^{11}$ Nikhef, Science Park, 1098 XG Amsterdam, The Netherlands

${ }^{12}$ LIGO, Massachusetts Institute of Technology, Cambridge, Massachusetts 02139, USA

${ }^{13}$ Instituto Nacional de Pesquisas Espaciais, 12227-010 São José dos Campos,

Sao Paulo, Brazil

${ }^{14}$ INFN, Gran Sasso Science Institute, I-67100 L'Aquila, Italy

${ }^{15}$ INFN, Sezione di Roma Tor Vergata, I-00133 Roma, Italy

${ }^{16}$ Inter-University Centre for Astronomy and Astrophysics, Pune 411007, India

${ }^{17}$ International Centre for Theoretical Sciences, Tata Institute of Fundamental Research, Bangalore 560012, India

${ }^{18}$ University of Wisconsin-Milwaukee, Milwaukee, Wisconsin 53201, USA

${ }^{19}$ Leibniz Universität Hannover, D-30167 Hannover, Germany

${ }^{20}$ Università di Pisa, I-56127 Pisa, Italy

${ }^{21}$ INFN, Sezione di Pisa, I-56127 Pisa, Italy

${ }^{22}$ Australian National University, Canberra, Australian Capital Territory 0200, Australia

${ }^{23}$ The University of Mississippi, University, Mississippi 38677, USA

${ }^{24}$ California State University Fullerton, Fullerton, California 92831, USA 
${ }^{25}$ LAL, Univ. Paris-Sud, CNRS/IN2P3, Université Paris-Saclay, F-91898 Orsay, France

${ }^{26}$ Chennai Mathematical Institute, Chennai 603103, India

${ }^{27}$ Università di Roma Tor Vergata, I-00133 Roma, Italy

${ }^{28}$ University of Southampton, Southampton SO17 1BJ, United Kingdom

${ }^{29}$ Universität Hamburg, D-22761 Hamburg, Germany

${ }^{30}$ INFN, Sezione di Roma, I-00185 Roma, Italy

${ }^{31}$ Albert-Einstein-Institut, Max-Planck-Institut für Gravitationsphysik, D-14476 Potsdam-Golm, Germany

${ }^{32}$ APC, AstroParticule et Cosmologie, Université Paris Diderot, CNRS/IN2P3, CEA/Irfu,

Observatoire de Paris, Sorbonne Paris Cité, F-75205 Paris Cedex 13, France

${ }^{33}$ Montana State University, Bozeman, Montana 59717, USA

${ }^{34}$ Università di Perugia, I-06123 Perugia, Italy

${ }^{35}$ INFN, Sezione di Perugia, I-06123 Perugia, Italy

${ }^{36}$ European Gravitational Observatory (EGO), I-56021 Cascina, Pisa, Italy

${ }^{37}$ Syracuse University, Syracuse, New York 13244, USA

${ }^{38}$ SUPA, University of Glasgow, Glasgow G12 8QQ, United Kingdom

${ }^{39}$ LIGO Hanford Observatory, Richland, Washington 99352, USA

${ }^{40}$ Wigner RCP, RMKI, H-1121 Budapest, Konkoly Thege Miklós út 29-33, Hungary

${ }^{41}$ Columbia University, New York, New York 10027, USA

${ }^{42}$ Stanford University, Stanford, California 94305, USA

${ }^{43}$ Università di Padova, Dipartimento di Fisica e Astronomia, I-35131 Padova, Italy

${ }^{44}$ INFN, Sezione di Padova, I-35131 Padova, Italy

${ }^{45}$ CAMK-PAN, 00-716 Warsaw, Poland

${ }^{46}$ University of Birmingham, Birmingham B15 2TT, United Kingdom

${ }^{47}$ Università degli Studi di Genova, I-16146 Genova, Italy

${ }^{48}$ INFN, Sezione di Genova, I-16146 Genova, Italy

${ }^{49}$ RRCAT, Indore Madhya Pradesh 452013, India

${ }^{50}$ Faculty of Physics, Lomonosov Moscow State University, Moscow 119991, Russia

${ }^{51}$ SUPA, University of the West of Scotland, Paisley PA1 2BE, United Kingdom

${ }^{52}$ University of Western Australia, Crawley, Western Australia 6009, Australia

${ }^{53}$ Department of Astrophysics/IMAPP, Radboud University Nijmegen, P.O. Box 9010, 6500 GL Nijmegen, The Netherlands

${ }^{54}$ Artemis, Université Côte d'Azur, CNRS, Observatoire Côte d'Azur, CS 34229 Nice cedex 4, France

${ }^{55}$ Institut de Physique de Rennes, CNRS, Université de Rennes 1, F-35042 Rennes, France

${ }^{56}$ Washington State University, Pullman, Washington 99164, USA

${ }^{57}$ Università degli Studi di Urbino "Carlo Bo," I-61029 Urbino, Italy

${ }^{58}$ INFN, Sezione di Firenze, I-50019 Sesto Fiorentino, Firenze, Italy

${ }^{59}$ University of Oregon, Eugene, Oregon 97403, USA

${ }^{60}$ Laboratoire Kastler Brossel, UPMC-Sorbonne Universités, CNRS, ENS-PSL Research University, Collège de France, F-75005 Paris, France

${ }^{61}$ Carleton College, Northfield, Minnesota 55057, USA

${ }^{62}$ Astronomical Observatory Warsaw University, 00-478 Warsaw, Poland

${ }^{63} \mathrm{VU}$ University Amsterdam, $1081 \mathrm{HV}$ Amsterdam, The Netherlands

${ }^{64}$ University of Maryland, College Park, Maryland 20742, USA

${ }^{65}$ Center for Relativistic Astrophysics and School of Physics, Georgia Institute of Technology, Atlanta, Georgia 30332, USA

${ }^{66}$ Laboratoire des Matériaux Avancés (LMA), CNRS/IN2P3, F-69622 Villeurbanne, France

${ }^{67}$ Université Claude Bernard Lyon 1, F-69622 Villeurbanne, France

${ }^{68}$ Università di Napoli "Federico II," Complesso Universitario di Monte S.Angelo, I-80126 Napoli, Italy

${ }^{69}$ NASA/Goddard Space Flight Center, Greenbelt, Maryland 20771, USA

${ }^{70}$ RESCEU, University of Tokyo, Tokyo 113-0033, Japan

${ }^{71}$ Tsinghua University, Beijing 100084, China

${ }^{72}$ Texas Tech University, Lubbock, Texas 79409, USA

${ }^{73}$ Pennsylvania State University, University Park, Pennsylvania 16802, USA

${ }^{74}$ National Tsing Hua University, Hsinchu City, 30013 Taiwan, Republic of China

${ }^{75}$ Charles Sturt University, Wagga Wagga, New South Wales 2678, Australia

${ }^{76}$ West Virginia University, Morgantown, West Virginia 26506, USA

${ }^{77}$ University of Chicago, Chicago, Illinois 60637, USA

${ }^{78}$ Caltech CaRT, Pasadena, California 91125, USA 
${ }^{79}$ Korea Institute of Science and Technology Information, Daejeon 305-806, Korea

${ }^{80}$ Università di Roma “La Sapienza," I-00185 Roma, Italy

${ }^{81}$ University of Brussels, Brussels 1050 Belgium

${ }^{82}$ Sonoma State University, Rohnert Park, California 94928, USA

${ }^{83}$ Center for Interdisciplinary Exploration and Research in Astrophysics (CIERA), Northwestern University, Evanston, Illinois 60208, USA

${ }^{84}$ University of Minnesota, Minneapolis, Minnesota 55455, USA

${ }^{85}$ The University of Melbourne, Parkville, Victoria 3010, Australia

${ }^{86}$ Institute for Plasma Research, Bhat, Gandhinagar 382428, India

${ }^{87}$ The University of Sheffield, Sheffield S10 2TN, United Kingdom

${ }^{88}$ The University of Texas Rio Grande Valley, Brownsville, Texas 78520, USA

${ }^{89}$ Università di Trento, Dipartimento di Fisica, I-38123 Povo, Trento, Italy

${ }^{90}$ INFN, Trento Institute for Fundamental Physics and Applications, I-38123 Povo, Trento, Italy

${ }^{91}$ Cardiff University, Cardiff CF24 3AA, United Kingdom

${ }^{92}$ Montclair State University, Montclair, New Jersey 07043, USA

${ }^{93}$ MTA Eötvös University, "Lendulet” Astrophysics Research Group, Budapest 1117, Hungary

${ }^{94}$ National Astronomical Observatory of Japan, 2-21-1 Osawa, Mitaka, Tokyo 181-8588, Japan

${ }^{95}$ School of Mathematics, University of Edinburgh, Edinburgh EH9 3FD, United Kingdom

${ }^{96}$ Indian Institute of Technology, Gandhinagar Ahmedabad Gujarat 382424, India

${ }^{97}$ University of Szeged, Dóm tér 9, Szeged 6720, Hungary

${ }^{98}$ Embry-Riddle Aeronautical University, Prescott, Arizona 86301, USA

${ }^{99}$ Tata Institute of Fundamental Research, Mumbai 400005, India

${ }^{100}$ INAF, Osservatorio Astronomico di Capodimonte, I-80131 Napoli, Italy

${ }^{101}$ University of Michigan, Ann Arbor, Michigan 48109, USA

${ }^{102}$ Rochester Institute of Technology, Rochester, New York 14623, USA

${ }^{103}$ NCSA, University of Illinois at Urbana-Champaign, Urbana, Illinois 61801, USA

${ }^{104}$ Universitat de les Illes Balears, IAC3-IEEC, E-07122 Palma de Mallorca, Spain

${ }^{105}$ University of Biatystok, 15-424 Biat ystok, Poland

${ }^{106}$ SUPA, University of Strathclyde, Glasgow G1 1XQ, United Kingdom

${ }^{107}$ IISER-TVM, CET Campus, Trivandrum Kerala 695016, India

${ }^{108}$ Canadian Institute for Theoretical Astrophysics, University of Toronto, Toronto, Ontario M5S 3H8, Canada

${ }^{109}$ Institute of Applied Physics, Nizhny Novgorod 603950, Russia

${ }^{110}$ Pusan National University, Busan 609-735, Korea

${ }^{111}$ Hanyang University, Seoul 133-791, Korea

${ }^{112}$ University of Adelaide, Adelaide, South Australia 5005, Australia

${ }^{113}$ NCBJ, 05-400 Świerk-Otwock, Poland

${ }^{114}$ IM-PAN, 00-956 Warsaw, Poland

${ }^{115}$ Monash University, Victoria 3800, Australia

${ }^{116}$ Seoul National University, Seoul 151-742, Korea

${ }^{117}$ The Chinese University of Hong Kong, Shatin, New Territories, Hong Kong SAR, China

${ }^{118}$ University of Alabama in Huntsville, Huntsville, Alabama 35899, USA

${ }^{119}$ University of Massachusetts-Amherst, Amherst, Massachusetts 01003, USA

${ }^{120}$ ESPCI, CNRS, F-75005 Paris, France

${ }^{121}$ Università di Camerino, Dipartimento di Fisica, I-62032 Camerino, Italy

${ }^{122}$ Southern University and A\&M College, Baton Rouge, Louisiana 70813, USA

${ }^{123}$ College of William and Mary, Williamsburg, Virginia 23187, USA

${ }^{124}$ Instituto de Física Teórica, University Estadual Paulista/ICTP South American Institute

for Fundamental Research, São Paulo São Paulo 01140-070, Brazil

${ }^{125}$ University of Cambridge, Cambridge CB2 1TN, United Kingdom

${ }^{126}$ IISER-Kolkata, Mohanpur, West Bengal 741252, India

${ }^{127}$ Rutherford Appleton Laboratory, HSIC, Chilton, Didcot, Oxon OX11 OQX, United Kingdom

${ }^{128}$ Whitman College, 345 Boyer Avenue, Walla Walla, Washington 99362 USA

${ }^{129}$ National Institute for Mathematical Sciences, Daejeon 305-390, Korea

${ }^{130}$ Université de Lyon, F-69361 Lyon, France

${ }^{131}$ Hobart and William Smith Colleges, Geneva, New York 14456, USA

${ }^{132}$ Janusz, Gil Institute of Astronomy, University of Zielona Góra, 65-265 Zielona Góra, Poland

${ }^{133}$ King's College London, University of London, London WC2R 2LS, United Kingdom

${ }^{134}$ Andrews University, Berrien Springs, Michigan 49104, USA

${ }^{135}$ Università di Siena, I-53100 Siena, Italy 
${ }^{136}$ Trinity University, San Antonio, Texas 78212, USA

${ }^{137}$ University of Washington, Seattle, Washington 98195, USA

${ }^{138}$ Kenyon College, Gambier, Ohio 43022, USA

${ }^{139}$ Abilene Christian University, Abilene, Texas 79699, USA

${ }^{\dagger}$ Deceased. 